Portland State University

PDXScholar

\title{
Mitochondrial Reactive Oxygen Species (ROS): Which ROS is Responsible for Cardioprotective Signaling?
}

Anders Olav Garlid

Portland State University

Follow this and additional works at: https://pdxscholar.library.pdx.edu/open_access_etds

Part of the Cell Biology Commons

Let us know how access to this document benefits you.

\section{Recommended Citation}

Garlid, Anders Olav, "Mitochondrial Reactive Oxygen Species (ROS): Which ROS is Responsible for Cardioprotective Signaling?" (2014). Dissertations and Theses. Paper 1641.

https://doi.org/10.15760/etd.1640

This Thesis is brought to you for free and open access. It has been accepted for inclusion in Dissertations and Theses by an authorized administrator of PDXScholar. Please contact us if we can make this document more accessible: pdxscholar@pdx.edu. 
Mitochondrial Reactive Oxygen Species (ROS):

Which ROS Is Responsible for Cardioprotective Signaling?

by

Anders Olav Garlid

A thesis submitted in partial fulfillment of the requirements for the degree of

Master of Science

in

Biology

Thesis Committee:

Jason Podrabsky, Chair

Stanley Hillman

Suzanne Estes

Portland State University

2014 


\begin{abstract}
Mitochondria are the major effectors of cardioprotection by procedures that open the mitochondrial ATP-sensitive potassium channel (mitoK $\left.\mathrm{ATP}_{\mathrm{A}}\right)$, including ischemic and pharmacological preconditioning. MitoK $\mathrm{K}_{\text {ATP }}$ opening leads to increased reactive oxygen species (ROS), which then activate a mitoK $\mathrm{ATP}$ associated $\mathrm{PKC} \varepsilon$, which phosphorylates mitoK $\mathrm{ATP}_{\mathrm{TP}}$ and leaves it in a persistent open state (Costa, ADT and Garlid, KD. Am J Physiol 295, H874-82, 2008). Superoxide $\left(\mathrm{O}_{2}{ }^{-}\right)$, hydrogen peroxide $\left(\mathrm{H}_{2} \mathrm{O}_{2}\right)$, and hydroxyl radical $\left(\mathrm{HO}^{\circ}\right)$ have each been proposed as the signaling ROS but the identity of the ROS responsible for this feedback effect is not known. Superoxide was excluded in earlier work on the basis that it does not activate $\mathrm{PKC} \varepsilon$ and does not induce mitoK $_{\text {ATP }}$ opening (20). To further examine the identity of the signaling ROS, respiring rat heart mitochondria were preincubated with ATP and diazoxide to induce the phosphorylation-dependent open state, together with agents that may interrupt feedback activation of mitoK $\mathrm{ATP}_{\mathrm{TP}}$ by ROS scavenging or by blocking ROS transformations. Swelling assays of the preincubated mitochondria revealed that dimethylsulfoxide (DMSO), dimethylformamide (DMF), deferoxamine, trolox, and bromoenol lactone (BEL) each blocked the ROS-dependent open state but catalase did not interfere with this step. The lack of a catalase effect and the inhibitory effects of agents acting downstream of $\mathrm{HO}^{\circ}$ excludes $\mathrm{H}_{2} \mathrm{O}_{2}$ as the endogenous signaling $\mathrm{ROS}$ and focuses attention on $\mathrm{HO}^{\circ}$. In support of the hypothesis that $\mathrm{HO}^{\circ}$ is required, we also found that $\mathrm{HO}^{\circ}$-scavenging by DMF
\end{abstract}


blocked cardioprotection by both ischemic preconditioning and diazoxide in the Langendorff perfused rat heart. $\mathrm{HO}^{\circ}$ itself cannot act as a signaling molecule, because its lifetime is too short and it reacts immediately with nearest neighbor phospholipids and proteins. Therefore, these findings point to a product of phospholipid peroxidation, such as hydroperoxy-fatty acids. Indeed, this hypothesis was supported by the finding that hydroperoxylinoleic acid ( $\mathrm{LAOOH})$ opens the ATP-inhibited mitoK $\mathrm{ATP}_{\text {ATP }}$ in isolated mitochondria. This effect was blocked by the specific PKC $\varepsilon$ inhibitor peptide $\varepsilon \bigvee_{1-2}$, showing that $\mathrm{LAOOH}$

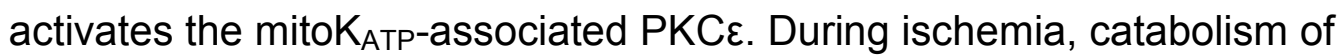
mitochondrial phospholipids is accelerated, causing accumulation of plasmalogens and free fatty acids (FA) in the heart by the action of calciumindependent phospholipases A2 (iPLA2). We first assessed the role of FAs and hydroxy FAs on mitoK $\mathrm{K}_{\text {ATP }}$ opening and cardioprotection. Swelling assays of isolated rat heart mitochondria showed that naturally formed free FAs inhibit mitoK $_{\text {ATP }}$ opening and that they are more potent inhibitors of the pharmacological open state of mitoK $\mathrm{ATP}_{\mathrm{T}}$ than the phosphorylation-dependent open state. That is, sustained mitoK $\mathrm{K}_{\mathrm{ATP}}$ opening induced by the phosphorylation-dependent feedback loop is more resistant to FA inhibition than direct mitoK $\mathrm{ATP}_{\text {opening by }}$ a potassium channel opener. Moreover, rat hearts perfused with micromolar concentrations of FA were resistant to cardioprotection by diazoxide or ischemic preconditioning. Racemic bromoenol lactone (BEL), a selective inhibitor of $\mathrm{iPLA} 2$, confers protection to otherwise untreated Langendorff perfused hearts by 
preventing ischemic FA release. To bring this story full circle, BEL blocks protection afforded by preconditioning and postconditioning by preventing the iPLA2-mediated release of $\mathrm{FAOOH}$ generated in the conditioned heart. $\mathrm{HO}^{\circ}$ resulting from mitoK $\mathrm{K}_{\text {ATP }}$ opening oxidizes polyunsaturated fatty acid components of the membrane phospholipids, resulting in a peroxidized side chain. FAOOH must be released in order to act on the mitochondrial $\mathrm{PKC} \varepsilon$, and this is achieved by the action of iPLA2. iPLA2 is essential for most modes of cardioprotection because it catalyzes the release of $\mathrm{FAOOH}$. This fully supports the hypothesis that the second messenger of cardioprotective ROS-mediated signaling is hydroperoxy fatty acid $(\mathrm{FAOOH})$, a downstream oxidation product of $\mathrm{HO}^{\circ}$. 


\section{Dedication}

I dedicate this thesis to my late mentor and friend, William Stanley, and to my parents, Keith Garlid and Randi Brannan, all of whom inspired me, kept me energized and put up with me through it all. 


\section{Acknowledgments}

The author expresses gratitude for the technical and administrative assistance of

Craig Semrad, Caitlin E. Pesout and Beau Sober and the guidance provided by Keith Garlid and Jason Podrabsky. 


\section{Table of Contents}

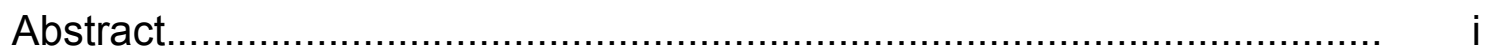

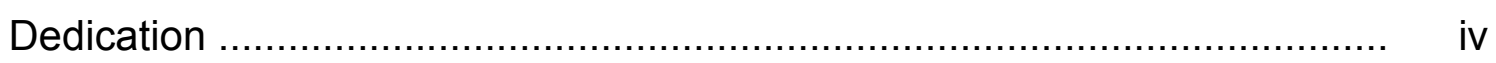

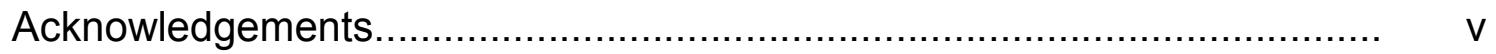

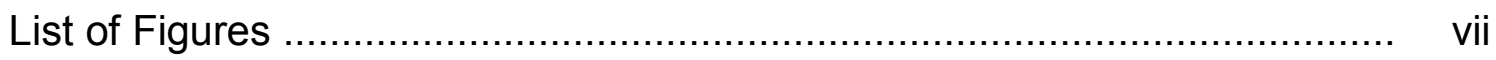

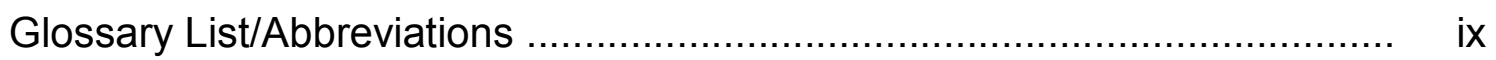

Chapter 1

Literature Review: From the Chemiosmotic Theory to Signaling in the Heart

Chapter 2

Mitochondrial Reactive Oxygen Species (ROS): Which ROS Signals Cardioprotection?

2-A. Abstract

2-B. Introduction

2-C. Research Design and Methods

2-D. Results 35

2-E. Discussion

Chapter 3

3-A. Abstract

3-B. Introduction 53

3-C. Research Design and Methods 57

3-D. Results

3-E. Discussion

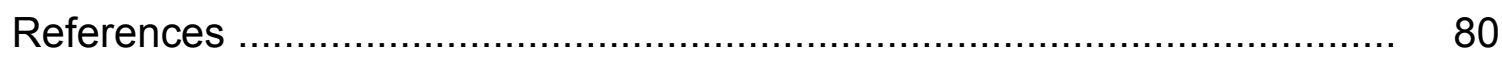

Appendix A. Publishing Permission . ................................. 91 


\section{List of Figures}

\section{Chapter 1:}

Fig. 1.1. (p. 1) The first three postulates of Mitchell's chemiosmotic theory.

Fig. 1.2. (p. 5) The mitochondrial potassium cycle.

Fig. 1.3. (p. 7) Flux (J/Jo) vs. membrane potential $(\Delta \Psi)$.

Fig. 1.4. (p. 10) MitoK $K_{A T P}$ regulation of VDAC permeability to nucleotides during ischemia and during high rates of ATP production.

Fig. 1.5. (p. 18) Intramitochondrial signaling and ROS generation.

Fig. 1.6. (p. 20) Effect of matrix $\mathrm{pH}$ on ROS production from complex I.

\section{Chapter 2:}

Fig. 2.1. (p. 25) Mitochondrial electron transport chain-derived ROS and formation of alkyl peroxl/alkoxyl radicals.

Fig. 2.2. (p. 26) Lipid peroxidation and release of hydroperoxy fatty acids.

Fig. 2.3. (p. 29) Isolated heart protocols.

Fig. 2.4. (p. 32) Preincubation protocol.

Fig. 2.5. (p. 35) ROS-dependent feedback activation of mito $K_{A T P}$

Fig. 2.6. (p. 36) Feedback activation of mito $K_{A T P}$ is blocked by 5hydroxydecanoate (5-HD) and $\varepsilon V_{1-2}$.

Fig. 2.7. (p. 37) Effects of agents that may block formation of the signaling ROS on feedback activation of mito $K_{A T P}$.

Fig. 2.8. (p. 39) Differential effects of MPG and Trolox on $\mathrm{H}_{2} \mathrm{O}_{2}$-dependent mitoK $K_{A T P}$ opening.

Fig. 2.9. (p. 39) Effects of agents of activation of $\mathrm{PKC} \varepsilon 1$ by $\mathrm{H}_{2} \mathrm{O}_{2}$.

Fig. 2.10. (p. 40) Dimethylformamide (DMF) blocks protection of the perfused heart by diazoxide and IPC.

Fig. 2.11. (p. 41) Effects of free radical reactants on mito $K_{A T P}$-dependent MPT inhibition.

\section{Chapter 3:}

Fig. 3.1. (p. 55) Lipid peroxidation and release of hydroperoxy fatty acids.

Fig. 3.2. (p. 61) 13-(S)-HODE inhibits mitoK $K_{A T P .}$

Fig. 3.3. (p. 63) Diazoxide exhibits a $\sim 25$ fold greater sensitivity to 13-(S)-HODE than PMA.

Fig. 3.4. (p. 64) Isolated heart protocol - HODE studies.

Fig. 3.5. (p. 64) 13-(S)-HODE blocks IPC and Dzx protection in isolated perfused rat heart. 
Fig. 3.6. (p. 65) Hydroperoxy fatty acid-dependent opening of mito $K_{A T P}$ is mediated via $P K C \varepsilon$.

Fig. 3.7. (p. 66) FAOOH confer protection to the same degree as IPC and diazoxide.

Fig. 3.8. (p. 67) Dual role of $\mathrm{Ca}^{2+}$-independent phospholipase A2 (iPLA2) action in mitochondria and cardioprotection.

Fig. 3.9. (p. 69) Inhibition of $\mathrm{Ca}^{2+}$-independent phospholipase A2 (iPLA2) demonstrates that $\mathrm{FAOOH}$ are necessary for signaling. 


\section{Glossary List/Abbreviations}

ETS

$F_{1}, F_{0}$-ATPase

$\mathrm{K}^{+} / \mathrm{H}^{+}$antiporter

MitoK $_{\text {ATP }}$

$\mathrm{Na}, \mathrm{K}-\mathrm{ATP}$ ase

$\Delta \Psi$

VDAC

ANT

Kir

SUR

ROS

$\mathrm{O}_{2}$ -

$\mathrm{H}_{2} \mathrm{O}_{2}$

$\mathrm{HO}^{\circ}$

FA

$\mathrm{FAOOH}$

PKC

GPCR

SOD

iPLA2

$\mathrm{KCO}$

IPC

MPG

Dzx

5-HD

$\varepsilon \mathrm{V}_{1-2}$

CCCP

BEL

Dfo

DMF

DMSO

Trolox

Catalase electron transport system

ATP synthase

inner membrane mitochondrial protein for potassium and

hydrogen exchange

mitochondrial ATP-sensitive $\mathrm{K}^{+}$channel

sodium-potassium adenosine triphosphatase $\left(\mathrm{Na}^{+}-\mathrm{K}^{+}\right.$pump)

membrane potential

voltage-dependent anion channels

adenine nucleotide translocator

inward-rectifying potassium channel

sulfonyl-urea receptor

reactive oxygen species

superoxide

hydrogen peroxide

hydroxyl radical

fatty acid

hydroperoxy fatty acid

protein kinase $\mathrm{C}$

$\mathrm{G}_{\mathrm{i}}$-protein coupled receptor

superoxide dismutase (converts $\mathrm{O}_{2}{ }^{--}$into $\mathrm{H}_{2} \mathrm{O}_{2}$ )

calcium-independent phospholipase A2

potassium channel opener

ischemic preconditioning

mercaptopropionyl glycine (thiolreductant, $\mathrm{HO}^{\bullet}$ scavenger)

diazoxide (potassium channel opener)

5-hydroxy decanoate (mitoK $\mathrm{K}_{\text {ATP }}$ channel inhibitor)

epsilon- $\mathrm{V}_{1-2}$ (PKCE inhibitor)

carbonyl cyanide m-chlorophenyl hydrazine (protonophore)

bromoenol lactone (iPLA2 inhibitor)

deferoxamine (iron scavenger)

dimethyl formamide (hydroxyl radical scavenger)

dimethylsulfoxide (hydroxyl radical scavenger)

chain breaking antioxidant, peroxyl radical scavenger

decomposes $\mathrm{H}_{2} \mathrm{O}_{2}$ into water and oxygen 


\section{Chapter 1: Literature Review}

"From the Chemiosmotic Theory to Signaling in the Heart"

\section{Mitochondria}

Mitochondria produce ATP by oxidative phosphorylation, which uses the chemical energy of substrate oxidation. The mechanism by which substrate oxidation is coupled to phosphorylation remained elusive for many years. The prevailing assumption held that there must be a high-energy chemical intermediate that couples these processes, but no such intermediate could be found. In 1961, Peter Mitchell proposed a novel hypothesis to explain the coupling phenomenon, completely independent of experimental evidence (98). Within a decade, his model was widely accepted, and in 1978 he was awarded the Nobel Prize for this massive accomplishment.

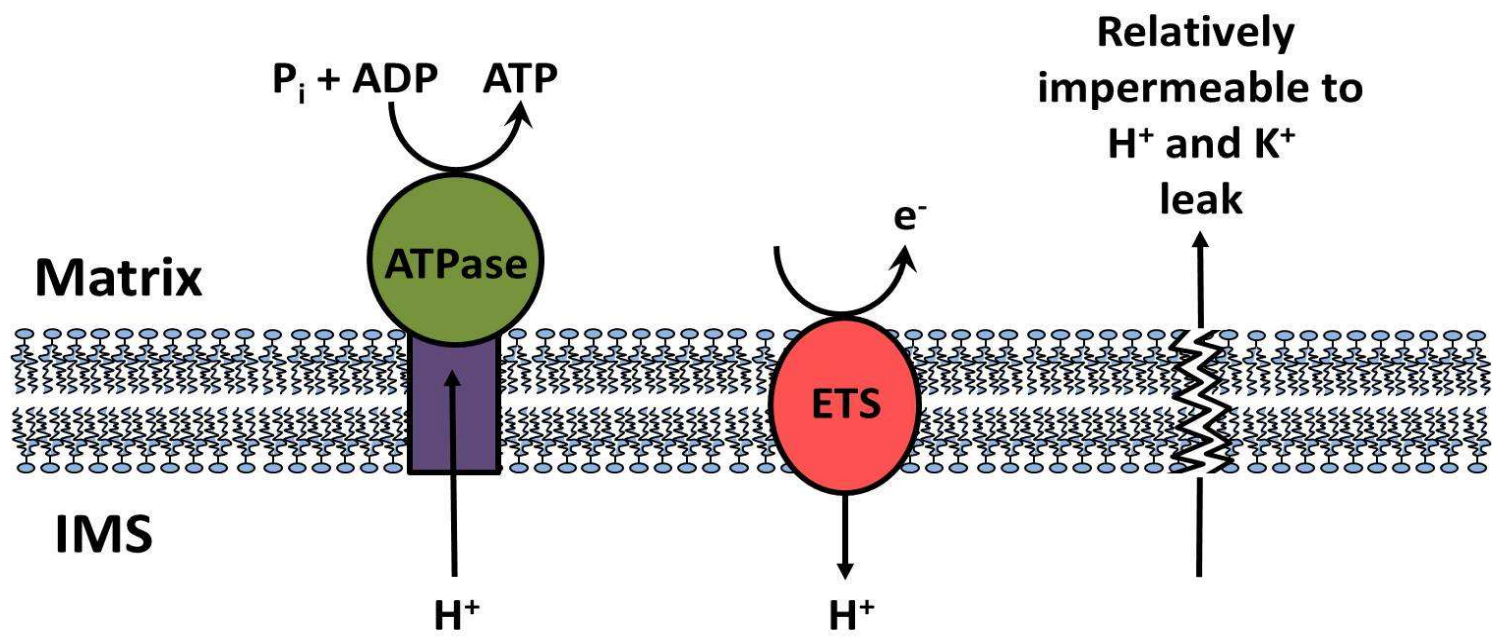

Fig. 1.1: The first three postulates of Mitchell's chemiosmotic theory.1) The vectoriallyoriented $\mathrm{F}_{1}, \mathrm{~F}_{0}$-ATPase pumps hydrogen ions $\left(\mathrm{H}^{+}\right)$out of the matrix to produce ATP from ADP with the addition of phosphate $\left(\mathrm{P}_{\mathrm{i}}\right)$. 2) The electron transport system (ETS) harnesses the chemical energy of substrate oxidation and drives protons out of the matrix. 3) The mitochondrial inner membrane is relatively impermeable to protons. Taken together, these three properties establish a kind of protonic battery that generates an electricochemical gradient across the membrane in the form of membrane potential $(\Delta \Psi)$. 


\section{Chemiosmotic Theory}

Mitchell's hypothesis is comprised of four basic postulates, three of which define the systems of the battery required for ATP synthesis and a fourth which describes how mitochondria must deal with the physiological stresses imposed by the chemiosmotic mechanisms.

Mitchell's first three postulates regarding ATP synthesis are as follows, and are depicted in Fig. 1.1: (Postulate 1 ) The $F_{1}, F_{0}-A T P a s e$ is a vectorially oriented enzyme that pumps hydrogen ions out of the mitochondrial matrix, using the energy of ATP hydrolysis. (Postulate 2) The electron transport system (ETS) is a series of vectorially oriented enzymes that harness the chemical energy of substrate oxidation and, through a series of oxidation-reduction reactions, drives protons out of the matrix. This action creates an electrochemical gradient for protons, which Mitchell called the protonmotive force. Because the $F_{1}, F_{0}$-ATPase is reversible, the protons pumped outward by the ETS can flow inward through the enzyme, coupling the synthesis of ATP to the inward translocation of protons. Thus, the protonmotive force is the missing connection that couples oxidation to phosphorylation.

In order for this system to synthesize ATP (Postulate 3), the membrane containing the $F_{1}, F_{0}-A T P a s e$ and the ETS enzymes must be relatively impermeable to ions, and especially to protons. The protonic battery would be short-circuited and no ATP would be synthesized if protons were permitted to leak across the membrane. The vectorial nature of the ETS and $F_{1}, F_{0}-A T P a s e$ and a relatively impermeable inner membrane were completely novel concepts at 
the time but they form the basis of our understanding of oxidativephosphorylation today (97).

The protonic battery described by the first three postulates generates a very high membrane potential $(\Delta \Psi)$ due to the constant extrusion of protons by the ETS. This will drive uptake of $\mathrm{K}^{+}$ions into the mitochondrial matrix so as to balance the charge between the IMS and the matrix. The influx of $\mathrm{K}^{+}$ions draws water into the matrix due to osmotic demands, causing its volume to increase at such a rate that the mitochondrion would rupture within minutes (48). Moreover, the tricarboxylic acid cycle requires uptake of substrate anions against their electrochemical gradient. To address these inherent physiological requirements, Mitchell proposed (Postulate 4) the existence of cation $/ \mathrm{H}^{+}$antiporters and anion exchangers that operate electroneutrally, thereby allowing essential metabolites to enter and excess cations to exit in order to maintain volume homeostasis (38, 97).

\section{Ion diffusion across the mitochondrial inner membrane}

lons, such as $\mathrm{K}^{+}$and $\mathrm{H}^{+}$, diffuse across the mitochondrial inner membrane at rates largely determined by the electrical gradient. The phospholipid bilayer presents a large energy barrier that ions must cross to gain access to the other side of the membrane and the permeability coefficient of the membrane is a function of this energy barrier. Experiments are consistent with the location of the energy barrier peak at the center of the inner membrane such that only ions with 
a high enough energy get access to the other side (44). This is the rate-limiting step of ion diffusion across the membrane (44). The probability of crossing this energy barrier is given by the Boltzmann distribution function, $e^{\left(-\frac{\Delta \tilde{\mu}_{p}}{R T}\right)}$, where $\Delta \tilde{\mu}_{p} \equiv \tilde{\mu}_{p}-\tilde{\mu}_{a q}$ is the Gibbs energy of the ion at the peak of the energy barrier $\left(\mu_{p}\right)$ relative to its value in the energy well at the surface of the membrane $\left(\mu_{a q}\right)$. The flux expression $J=f P\left(C_{1 o} e^{\frac{u}{2}}-C_{2 o} e^{-\frac{u}{2}}\right)$ is derived from this probability function to quantify the diffusion of cations across the inner membrane. Here, $u$ is the reduced voltage $(z F \Delta \psi / R T)$, divided by 2 to account for the fact that the barrier is at the center of the membrane, $C_{10}$ and $C_{2 o}$ are bulk aqueous concentrations at the outer and inner surfaces of the inner membrane, respectively, $f$ is the surface partition coefficient, and $P$ is the permeability constant. The second term in this equation accounts for backflux of cations out of the matrix. But the high membrane potential renders backflux negligible and the second term can be dropped, leaving a simpler function, $J=f P\left(C_{10} e^{\frac{u}{2}}\right)(44)$.

Note that at high membrane potentials such as those seen in mitochondria, ion-diffusion is minimally affected by concentration gradients across the membrane. Note as well that all diffusing cations face the same rate limiting step of crossing the energy barrier. These predictions were confirmed by the Garlid lab with the demonstration that TEA ${ }^{+}$and $\mathrm{H}^{+}$diffusion across the mitochondrial inner membrane are quantitatively identical, differing only in their permeability coefficient. Therefore, $\mathrm{H}^{+}$ions are transported in the same way as 
cations in general and there is apparently no unique mechanism for their diffusion across the inner mitochondrial membrane (44).

\section{Potassium cycle $\left(\mathrm{K}^{+}\right.$-cycle)}

The $\mathrm{K}^{+}$-cycle depicted in Fig. 1.2 describes the flux of potassium across the inner membrane and the associated processes of electron transport (ETS) and anion flux via the $\mathrm{Pi}^{-} / \mathrm{H}^{+}$symporter. Although the inner membrane is relatively impermeable to ions, inward leak of $\mathrm{K}^{+}$does occur at significant rates. Net uptake of $\mathrm{K}^{+}$at the expense of proton ejection by the ETS will drive net uptake of $\mathrm{P}_{\mathrm{i}}$ and other ions. This salt uptake, in turn, will drive net uptake of water, with matrix swelling. Therefore, this inward $\mathrm{K}^{+}$leak, if left unchecked, will result in excessive matrix swelling and eventual lysis of the mitochondrion $(37,48)$.

The inward flux of $\mathrm{K}^{+}$driven by the extrusion of $\mathrm{H}^{+}$ions during electron transport alkalinizes the matrix, and the resulting $\mathrm{pH}$ gradient causes $\mathrm{Pi}^{-}$and $\mathrm{H}^{+}$

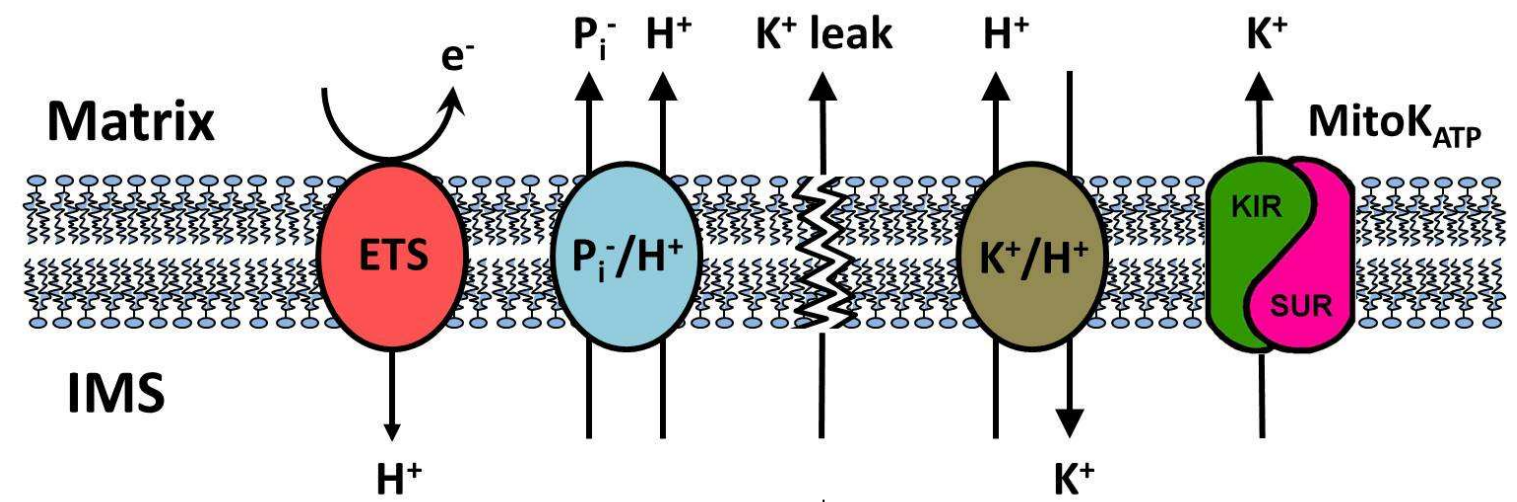

Fig. 1.2: The mitochondrial potassium cycle. The $\mathrm{K}^{+}$-cycle is comprised of the electron transport system (ETS), which sets up the electrochemical gradient in the form of the protonmotive force. The $\mathrm{P}_{\mathrm{i}}^{-} / \mathrm{H}^{+}$symporter $\left(\mathrm{P}_{\mathrm{i}}^{-} / \mathrm{H}^{+}\right)$provides the matrix with necessary anions and the $\mathrm{K}^{+} / \mathrm{H}^{+}$antiporter ejects $\mathrm{K}^{+}$to avoid excessive matrix swelling due to potassium leak $\left(\mathrm{K}^{+}\right.$ leak). The mitochondrial ATP-sensitive $\mathrm{K}^{+}$channel (mitoK $\mathrm{K}_{\mathrm{ATP}}$ ) is responsible for preventing matrix contracture during high work states by importing $\mathrm{K}^{+}$into the matrix. 
to enter through the $\mathrm{P}_{\mathrm{i}}{ }^{-} / \mathrm{H}^{+}$symporter. Additionally, as ion concentrations change with the rush of $\mathrm{K}^{+}$into the matrix, water is osmotically obligated to accompany the $\mathrm{K}^{+}$salt, causing matrix swelling. This sets up the physiological necessity of the ion exchange systems described by Mitchell's fourth postulate. Thus, the $\mathrm{K}^{+} / \mathrm{H}^{+}$antiporter must eject excess $\mathrm{K}^{+}$in exchange for a proton to maintain electroneutrality (48). Moreover, it must eject only the excess $\mathrm{K}^{+}$, because matrix $\mathrm{K}^{+}$is the primary osmolyte of the matrix.

Mitchell and Moyle initially uncovered evidence for $\mathrm{Na}^{+} / \mathrm{H}^{+}$and $\mathrm{K}^{+} / \mathrm{H}^{+}$ antiport by measuring swelling in sodium acetate $(\mathrm{NaOAc})$ and potassium acetate (KOAc) media (48). However, while $\mathrm{Na}^{+} / \mathrm{H}^{+}$exchange was robust, $\mathrm{K}^{+} / \mathrm{H}^{+}$ exchange was barely detectable and not sufficient to compensate for $\mathrm{K}^{+}$leak. Direct evidence for $\mathrm{K}^{+} / \mathrm{H}^{+}$antiport was provided by Garlid (42), who showed that swelling induces electroneutral potassium efflux (42). Mitochondria retain matrix $\mathrm{K}^{+}$in isotonic sucrose, which is too large to cross the inner membrane. In the hypotonic sucrose media, water enters the matrix in an attempt to dilute solute concentrations and achieve an osmotic balance, causing matrix swelling. The mitochondria respond by rapidly ejecting $\mathrm{K}^{+}$from the matrix in a passive and electroneutral manner $(42,48)$.

Several possible mechanisms exist for the regulation of this antiporter. The $\mathrm{Mg}^{2+}$ Carrier Brake Hypothesis states that the mitochondrial $\mathrm{K}^{+} / \mathrm{H}^{+}$antiporter is always partially inhibited by $\mathrm{Mg}^{2+}$ on the matrix side of the enzyme and that it uses small changes in free $\mathrm{Mg}^{2+}$ concentrations to regulate matrix volume (39). Matrix $\mathrm{Mg}^{2+}$ content is relatively stable but ion concentrations decrease by 
dilution when the matrix swells and $\mathrm{Mg}^{2+}$ activity is further decreased by the formation of complexes with incoming anions such as citrate and phosphate. This lowers the inhibition on the $\mathrm{K}^{+} / \mathrm{H}^{+}$antiporter, allowing ejection of $\mathrm{K}^{+}$and a return to normal volume. This mechanism provides high sensitivity to changes in matrix volume and prevents futile cycling of $\mathrm{K}^{+}$by tuning the $\mathrm{K}^{+} / \mathrm{H}^{+}$ antiporter to match $\mathrm{K}^{+}$uniport.

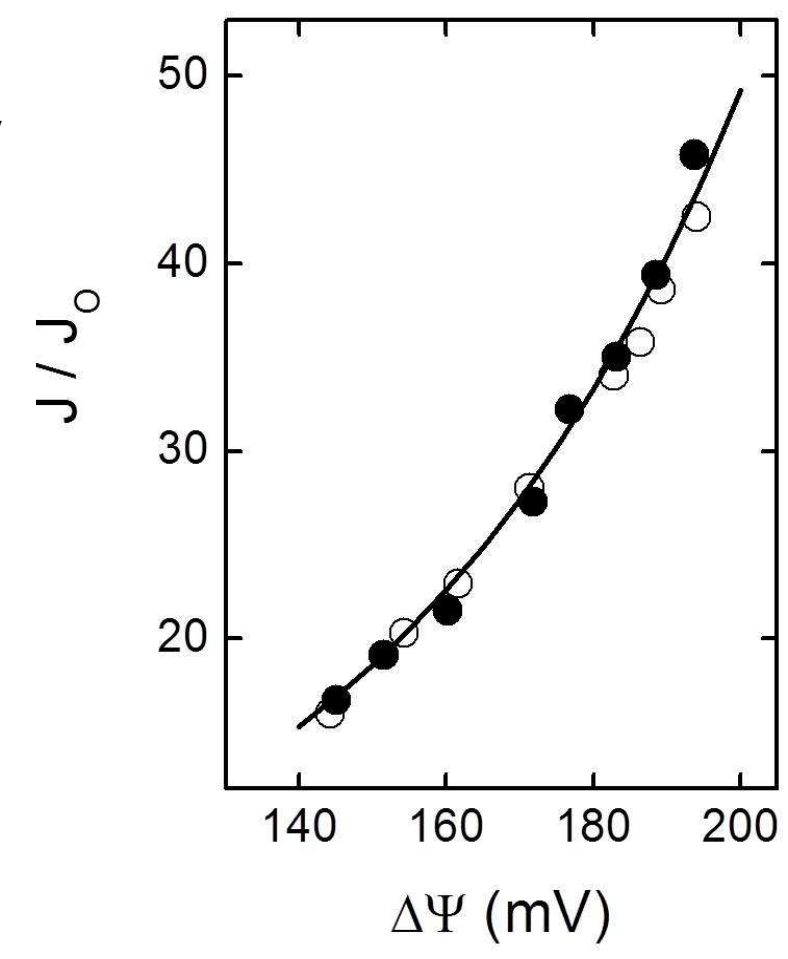

Fig. 1.3: Flux $\left(\mathrm{J} / \mathrm{J}_{0}\right)$ vs. membrane potential $(\Delta \Psi)$. The exponential relationship between $\Delta \Psi$ and $\mathrm{J} / \mathrm{J}_{0}$ means that a very small change in $\Delta \Psi$ results in a very large change in flux.

$\mathrm{K}^{+} / \mathrm{H}^{+}$antiport is also

inhibited by protons on the matrix side; $\mathrm{H}^{+}$concentration decreases below inhibitory concentration as the matrix swells and is alkalinized by the $\mathrm{K}^{+}$for $\mathrm{H}^{+}$ exchange during electron transport (39). Under experimental conditions with no $\mathrm{Mg}^{2+}$ present, matrix volume still regulates $\mathrm{K}^{+} / \mathrm{H}^{+}$exchange by an unknown mechanism. This may be due to other inhibitory ions or conformational changes experienced by the enzyme as the membrane is stretched $(39,48)$.

\section{Mitochondrial ATP-sensitive potassium channel (mitoK $\mathrm{ATP}_{\text {) }}$}

$\mathrm{A} \mathrm{K}^{+}$uniporter was reconstituted from mitochondria by Mironova et al. (96) and Diwan et al. (26) but its regulation and amino acid sequences were not 
determined. This raised a new problem - mitochondria were already having to deal with $\mathrm{K}^{+}$leak, why would they need a $\mathrm{K}^{+}$channel?

It was proposed that a $\mathrm{K}^{+}$channel is needed for volume homeostasis in high work states (48). ATP production and oxygen consumption in the heart can increase up to 8-fold of their normal levels in a high work state (40). Higher rates of ATP synthesis arise from greater $\mathrm{H}^{+}$ejection and the greater $\mathrm{H}^{+}$current will cause electrical potential $(\Delta \Psi)$ to drop based on the internal resistance of the electron transport system. The relationship between flux and $\Delta \Psi$ is exponential, as seen in Fig. 1.3. A small change in membrane potential results in a very large change in flux $(48,107)$. Therefore, under high work states and reduced $\Delta \Psi, \mathrm{K}^{+}$ flux is greatly diminished and the mitochondrial matrix will contract, that is, the matrix volume will decrease. The $\mathrm{K}^{+} / \mathrm{H}^{+}$antiporter senses the reduced volume by the mechanisms discussed in the previous section and slows $\mathrm{K}^{+}$ejection to establish a lower steady state matrix volume.

While this prevents matrix collapse, the matrix is still contracted, and this compromises the efficiency of energy transfer in heart due to the larger intermembrane space. This limits nucleotide transport through voltage-dependent anion channels (VDAC) across the outer membrane (28), and VDAC has been shown to control outer membrane permeability to ADP and ATP (122). VDAC exists in a low-conductance state in the heart that is not highly permeable to nucleotides. Creatine and creatine phosphate mediate energy transfers between mitochondria and cytosol through mitochondrial creatine kinase (123). Creatine 
kinase spans the intermembrane space in an octameric arrangement, bridging the gap between the outer membrane VDAC and inner membrane adenine nucleotide translocator (ANT), as shown in Fig. $1.4(127,147)$. Creatine kinase has been shown to bind to VDAC (126) and Garlid et al. hypothesized that it is this interaction that confers the low-conductance state to VDAC, preventing the outer membrane from being overly permeable to nucleotides (46). This binding requires a narrow intermembrane space to allow for a tight interaction between the octamer and VDAC. However, high work states lead to matrix contraction due to the decrease in $\triangle \Psi$ and creatine kinase would dissociate from VDAC, leading to higher ATP and ADP conductance at the outer membrane (46). The exponential relationship between flux and membrane potential sets up a situation in which effective energy transfer is compromised by matrix contraction at precisely the moment that it is needed most.

The exponential relationship between $\mathrm{K}^{+}$flux and its driving force, $\Delta \Psi$, demonstrates the need for another volume regulation channel that imports $\mathrm{K}^{+}$ into the matrix to prevent contraction when $\Delta \Psi$ is decreased under high work states. The mitochondrial ATP-sensitive potassium channel (mitoK $\left.\mathrm{K}_{\mathrm{ATP}}\right)$ adds a conductance pathway that is parallel to $\mathrm{K}^{+}$leak to restore matrix volume (48). It might be expected that $\Delta \Psi$ would decrease with this additional pathway for $\mathrm{K}^{+}$ transport, reducing $\mathrm{K}^{+}$flux even further and exacerbating the problem of matrix contraction. However, mitoK $\mathrm{K}_{\text {ATP }}$ opening has been shown to reduce $\Delta \Psi$ by only about $1 \mathrm{mV}$, which is relatively insignificant in the normal physiological state; 
mitochondria typically experience membrane potentials around $140-180 \mathrm{mV}(37$, 40).

MitoK $_{\text {ATP }}$ was reconstituted by the Garlid lab, showing that the inner membrane contains an ATP-dependent $\mathrm{K}^{+}$channel $(62,66,116)$. $\mathrm{K}_{\text {ATP }}$ channels are comprised of a complex of two different proteins, including an inwardlyrectifying potassium channel (Kir) subunit and the sulfonylurea receptor (SUR) (69). Four of the Kir proteins are thought to form the $K_{\text {ATP }}$ channel pore and the SUR is responsible for regulation of the channel and its sensitivity to ATP and pharmacologic agents $(1,60)$. In 2012, Brian Foster et al. showed that the ROMK channel, another ATP-dependent potassium channel (Kir1.1), is a molecular
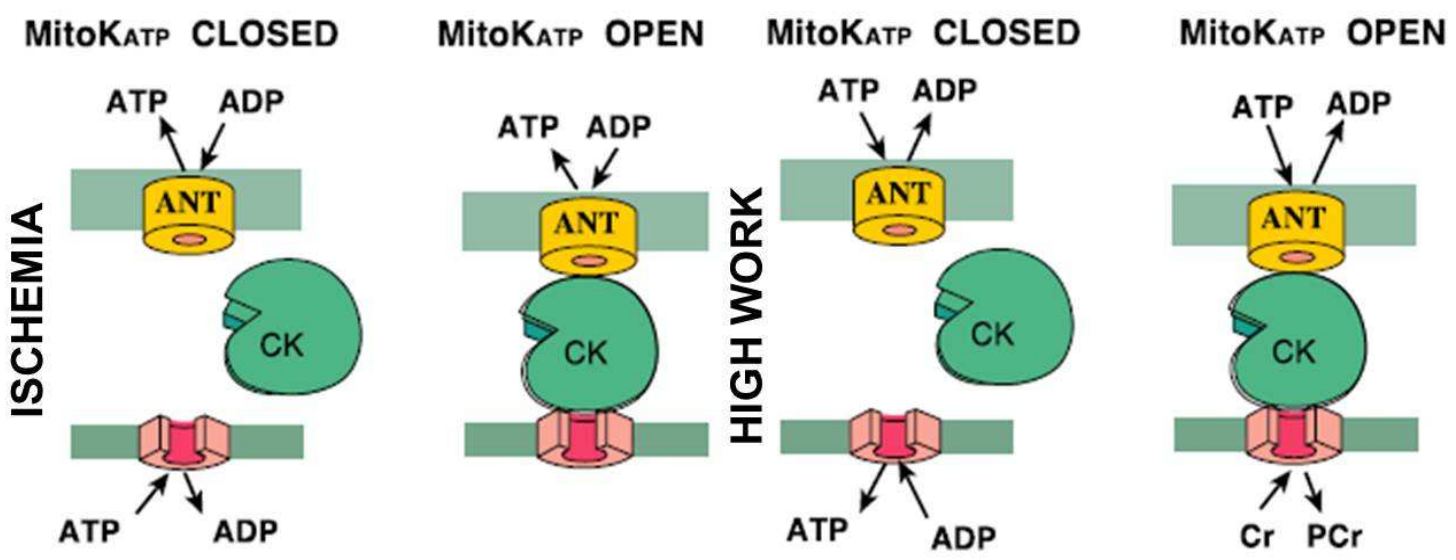

Fig. 1.4: Mito $K_{A T P}$ regulation of VDAC permeability to nucleotides during ischemia and during high rates of ATP production. $\triangle \Psi$ is supported by ATP hydrolysis during ischemia and by electron transport during the high work-state. Both of these stresses will cause a decrease in $\Delta \Psi$, resulting in reduced uptake of $\mathrm{K}+$ salts and water, contraction of the matrix, and expansion of the IMS. This structural change can be prevented by a compensatory increase in $\mathrm{K}+$ conductance mediated by opening mitoK $_{\text {ATP }}$. If mitoK $\mathrm{K}_{\text {ATP }}$ is blocked or does not open, the IMS expansion results in increased outer membrane permeability to ATP and ADP. During ischemia, this means that all of cellular ATP is available for hydrolysis by mitochondria, with consequent degradative loss of adenine nucleotides, and, ultimately, unavailability of ADP for rephosphorylation upon reperfusion. During the high work state, increased VDAC permeability to ADP and ATP constitutes a diversionary leak in the system away from the more efficient metabolic channeling through mitochondrial creatine kinase ("CK"). Consequently, if mitoK is blocked, mitochondria cannot supply ATP at the high rates required, and the heart cannot respond to inotropic stress. From Garlid et al., 2003 (46). 
subcomponent of the mitoK $\mathrm{K}_{\text {ATP }}$ channel (34).

MitoK $_{\text {ATP }}$ activity can be measured by light scattering, taking advantage of volume changes resulting from ion and water flux across the inner membrane ( 9 , 43). The amount of light scattered by suspended mitochondria is a function of matrix volume (136), where an increase in volume accompanies a decrease in the light scattering signal intensity (9). Mitochondria were first reported to take up water with decreasing osmolality of their surrounding solution by Claude in 1946 . They have since been shown to act as good osmometers, following the equation

$$
W_{m}=W_{b}+\frac{S_{o}}{\phi}
$$

where $W_{m}$ is matrix water content ( $\mathrm{mg}$ of $\mathrm{H}_{2} \mathrm{O} / \mathrm{mg}$ of protein), $W_{b}$ is osmotically inactive water, $S_{o}$ is water solute content (nosmol/mg), and $\phi$ is the osmolality of the containing medium (nosmol/mg of $\mathrm{H}_{2} \mathrm{O}$ ) (41). Direct measurement of matrix volume is an impractical method for solving this equation for rates of solute transport. Light scattering provides a reliable method by which to address this issue.

\section{Ischemia and cardioprotection}

Cardiovascular diseases are the leading causes of morbidity and mortality in man and ischemic heart disease is the leading cause of death in developed countries. Ischemia is the loss of blood flow to an organ, and extended periods of ischemia result in a large amount of cell death in the affected area, known as infarction, due to the lack of necessary nutrients (29). However, the damage 
does not present itself during the ischemic phase but rather upon reperfusion. The influx of fresh blood to the heart following a heart attack brings with it a large concentration of oxygen that quickly forms damaging reactive oxygen species. These proceed to overload antioxidant systems and cause irreparable damage to cardiac tissue. Preventive measures or medications that could render the heart resistant to ischemia or reperfusion injury following myocardial infarction would be an ideal mechanism for reducing the morbidity from heart disease. Our main method for reducing damage is to reperfuse the heart as quickly as possible, but more work must be done to salvage the myocardium during the acute phase of ischemia.

\section{Cardioprotection by ischemic preconditioning and postconditioning}

In1986, Murry et al. demonstrated that the heart could be conditioned with a brief period of ischemia prior to a longer period of ischemia, thus rendering the myocardium less susceptible to ischemic injury (102). A typical perfused heart can present up to $80 \%$ infarction of the myocardium after being subjected to an experimentally-induced heart attack or "index ischemia". "Ischemic preconditioning" (IPC) drastically reduced the infarct size to just $25 \%$ compared to untreated hearts and has since been studied extensively to determine the mechanism behind this intrinsic form of cardioprotection (29). Ischemic postconditioning (155) operates in a similar manner, restricting the flow of blood to the heart upon reperfusion through short bursts of reperfusion and ischemia prior to a full reperfusion. 
Cardioprotection by potassium channel openers: Cardioprotection can be achieved through preconditioning with potassium channel openers (KCOs) such as cromakalim, which mimic the protective effects of IPC. This was first observed in 1987 by Gross et al., using the KCO nicorandil, although it had not yet been classified as such (56). This class of drugs was first intentionally explored for their ability to confer protection by Grover et al. (61) and many more were demonstrated to have these effects in all animal models. $\mathrm{K}_{\mathrm{ATP}}$ channel blockers such as glibenclamide and 5-hydroxydecanoate (5-HD) abolish the protection afforded by cromakalim. That KCOs mimic the cardioprotective effect of IPC implicates $\mathrm{K}_{\text {ATP }}$ channels as the mechanism of action of IPC. Accordingly, Gross et al. demonstrated that KCO inhibitors block the protective effects of IPC. These results imply that $\mathrm{K}_{\mathrm{ATP}}$ channels are necessary for IPC and, presumably, for cardioprotection in general.

KCO protection by mitoK $\mathrm{ATP}_{\mathrm{A}}$ : The protective effects of KCOs were universally attributed to sarcolemmal $\mathrm{K}_{\mathrm{ATP}}$ channel (sarcK $\mathrm{K}_{\mathrm{ATP}}$ ) opening and a resultant "cardioplegic" effect (49). SarcK ATP channel opening was thought to mimic IPC by causing a reduction in action potential duration (APD) that would reduce $\mathrm{Ca}^{2+}$ influx into the cytosol and result in cardioplegia, a temporary reduction or cessation of cardiac activity. However, KCOs do not effect the contractility of the heart muscle or result in any form of negative isotropy seen in the cardiodepression associated with a cardioplegic effect (57). It was soon demonstrated that the cardioprotective effect of KCOs is not correlated to APD 
shortening $(58,59,151)$. Furthermore, vasodilation using KCOs is minimal, suggesting that this effect is not important for cardioprotection.

The lack of cardiodepression, APD shortening and vasodilation suggests that the target of KCOs is distinct from sarcolemmal $\mathrm{K}_{\mathrm{ATP}}(49)$. There are $\mathrm{K}_{\mathrm{ATP}}$ channels expressed in the mitochondrial membrane, mitoK $\mathrm{ATP}_{\text {, which regulate }}$ mitochondrial volume and energetics (70) and are opened by KCOs in their cardioprotective concentration range (52). Garlid proposed that mitoK $\mathrm{ATP}_{\text {and }}$ ant sarcK $_{\text {ATP }}$ is responsible for cardioprotection (37).

The role of mitoK $\mathrm{K}_{\mathrm{ATP}}$ channels in cardioprotection was confirmed by Garlid et al. with a series of experiments using diazoxide and cromakalim (49). Cromakalim is an effective cardioprotective agent that opens both sarcolemmal $\mathrm{K}_{\text {ATP }}$ (sarcK $\mathrm{K}_{\text {ATP }}$ ) and mitochondrial $\mathrm{K}_{\mathrm{ATP}}$ (mitoK $\mathrm{K}_{\mathrm{ATP}}$ ) with equal sensitivities. Diazoxide, on the other hand, opened bovine cardiac mitoK $\mathrm{ATP}_{\mathrm{A}}$ at a concentration 2000 times lower than in sarcK $\mathrm{K}_{\text {ATP }}$, making it a very useful tool for separating the effects of sarcK $\mathrm{K}_{\text {ATP }}$ and mitoK $\mathrm{K}_{\text {ATP }}(50)$. To that end, the same degree of cardioprotection using cromakalim was attained with diazoxide at concentrations well below its $\mathrm{k}_{1 / 2}$ for sarcK $\mathrm{K}_{\mathrm{ATP}}$ opening, strongly suggesting mediation by mitoK $\mathrm{K}_{\mathrm{ATP}}$ (49). Furthermore, diazoxide is a potent $\mathrm{K}_{\mathrm{ATP}}$ opener and effective cardioprotectant, but it has a weak APD shortening effect and it is a less potent vasodilator than cromakalim. These results support the conclusion that vasodilation and APD shortening are not correlated with the cardioprotective effects of KCOs or IPC (49). 
Further evidence for mitochondrial involvement in cardioprotection was obtained using the $\mathrm{K}_{\text {ATP }}$ channel inhibitor 5-HD. While 5-HD is an effective inhibitor of mitoK $\mathrm{K}_{\mathrm{ATP}}$ opening by cromakalim, it does not inhibit sarcK $\mathrm{K}_{\mathrm{ATP}}$ opening (94), providing another useful tool with which to distinguish the two $\mathrm{K}_{\text {ATP }}$ channels. Protection by cromakalim and diazoxide were inhibited by 5-HD with no effect on their APD shortening activity (94). Taken together, these results demonstrate that it is not sarcolemmal but mitochondrial $\mathrm{K}_{\mathrm{ATP}}$ channels that mediate cardioprotection by KCOs (49). Ischemic preconditioning is also blocked by $\mathrm{KCO}$ inhibitors, including $5-\mathrm{HD}$, indicating that the endogenous signaling mechanism of IPC is mediated by mitoK ATP $_{\text {as }}$ well $(46,49,50,52)$. The discovery that mitoK $\mathrm{K}_{\text {ATP }}$ is the target for potassium channel openers was a breakthrough that brought the focus of cardioprotection and its signaling mechanisms to the level of the mitochondrion.

\section{Cardioprotective signaling and $G_{i}$ protein-coupled receptors (GPCR)}

All forms of cardioprotection operate via mitochondrial $\mathrm{K}_{\mathrm{ATP}}$, including the intrinsic signaling mechanisms behind ischemic preconditioning. This process involves receptor-mediated signal formation at the cell membrane, leading to an intracellular signaling cascade that opens mitoK $_{\text {ATP. }}$ Ischemic preconditioning utilizes adenosine (90), bradykinin (146), and opioid (128) signaling acting in concert to confer protection to the ischemic heart. IPC can be mimicked pharmacologically by perfusing the heart with any one of these trigger substances and inhibition of any one of the adenosine, bradykinin, or opioid 
receptors blocks the protective effects of IPC (29). However, adding subsequent cycles of IPC can restore the protective effects, leading Goto et al. to propose that the three pathways operate in an additive manner to achieve a hypothetical protective threshold (55). If one receptor is inhibited and the heart is given further cycles of IPC, the two active receptors are able to reach this threshold and protect the heart from the injury that results from a longer period ischemia.

Each of these trigger substances acts through $G_{i}$ protein-coupled receptors (GPCR) and, according to the multiple trigger theory, all three must converge on a single target enzyme (29). Downey et al. determined that protein kinase $C(P K C)$ inhibitors reversed the protection afforded by all of these substances and proposed that the common target may be PKC (29). Activation of nearly any GPCR in the heart with agonists such as catecholamines, angiotensin II, or endothelin can mimic IPC. However, inhibiting any one of these other GPCRs does not block IPC as with adenosine, bradykinin, and opioid inhibitors (29). These three $\mathrm{G}_{\mathrm{i}}$-protein coupled receptors are the major players in signaling for ischemic preconditioning. Adenosine interacts directly with PKC through phospholipases while bradykinin and opiods trigger more complex pathways, ending up at the same target (29).

Costa et al. determined that protein kinase $G$ (PKG) is the terminal kinase in the GPCR signaling cascade triggered by IPC as well as the pharmacological agents that mimic protection (17). PKG and cyclic GMP (cGMP) added together to isolated mitochondria opens the mitoK $\mathrm{K}_{\mathrm{ATP}}$ channel with the same effect as a KCO such as diazoxide or cromakalim. 
MitoK $_{\text {ATP }}$ has been invoked by Pain et al. to be involved in the "trigger phase" of the IPC signaling pathway (111). Receptor stimulation leads to mitoK $_{\text {ATP }}$ opening, resulting in the formation of mitochondrial reactive oxygen species (ROS) which activate PKC. This leads to a form of protective "memory" that remains for about an hour after the preconditioning is complete or after the trigger substance has been washed out $(20,144)$. ROS are typically viewed as a damaging substance and, in many cases, they are. However, ROS also serve a valuable role in signaling as second messengers of many systems; they are required, for example, for cardioprotective signaling. The protection from diazoxide and ischemic preconditioning is abolished when a ROS scavenger is included $(6,32,139)$. Exposure to ROS also mimics the protective effects of IPC in a PKC-dependent manner $(6,139)$.

The mechanisms behind cardioprotective signaling and the increase in reactive oxygen species were still not well understood, but identifying mitoK $\mathrm{K}_{\mathrm{ATP}}$ as the target for signaling provided a focus for continued research.

\section{MitoK $_{\text {ATP }}$ and ROS}

All forms of cardioprotection operate through mitoK $\mathrm{ATP}_{\mathrm{A} \text {. Opening mitoK }}$ ATP causes an influx of $\mathrm{K}^{+}$and osmotically-obligated water, leading to mitochondrial matrix swelling and an increase in matrix $\mathrm{pH}$, as shown in Fig. 1.5. MitoK $\mathrm{ATP}_{\mathrm{A}}$ opening also leads to an increase in the level of ROS. Andrukhiv et al. demonstrated that mitoK $\mathrm{K}_{\mathrm{ATP}}$ opening by the KCOs diazoxide and cromakalim increased ROS production by $40-50 \%$. This effect can be blocked by the 
mitoK $_{\text {ATP }}$ channel blocker 5-HD (2). This increase in ROS was also shown to inhibit the mitochondrial permeability transition (MPT), thus preventing cell death (18). The high levels of calcium, phosphate and damaging ROS that persist after prolonged ischemia cause MPT to open, resulting in pathogenic necrotic cell death $(22,25,79)$. ROS arising from mitoK $\mathrm{ATP}$ opening activate a PKC $\varepsilon$ pool on the inner side of the inner membrane that is closely associated with MPT and prevents it from opening, thus preventing cell death.

While it was evident that mitochondrial ROS were necessary for

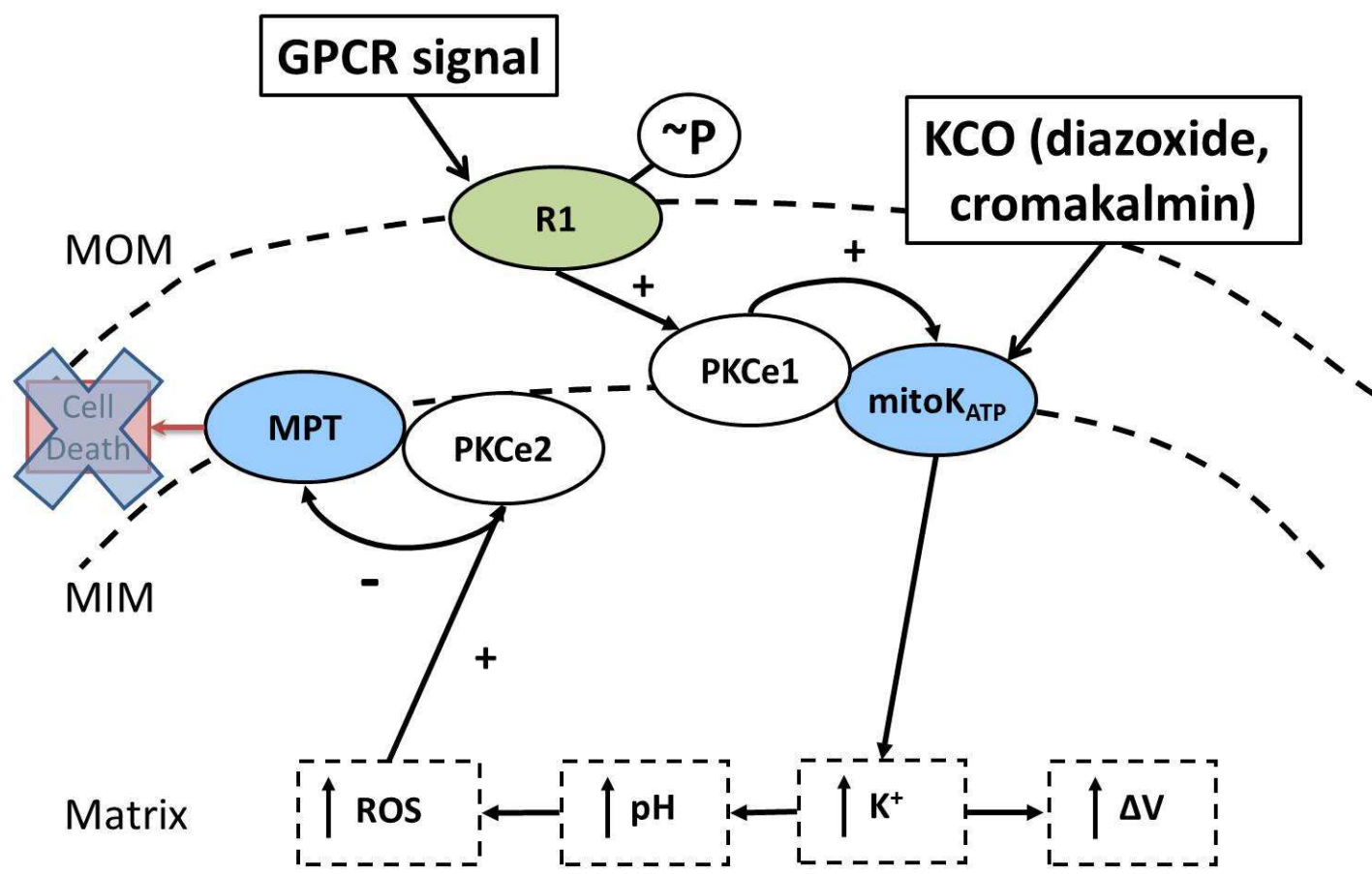

Fig. 1.5: Intramitochondrial signaling and ROS generation. The unidentified receptor R1 on the mitochondrial outer membrane (MOM) is phosphorylated by an intracellular GPCR signal. R1 then activates a pool of PKC $\varepsilon$ on the outer face of the inner membrane (MIM), which opens mitoK $_{\text {ATP }}$ by phosphorylation. Potassium channel openers (KCOs) such as diazoxide or cromakalim activate mitoK $\mathrm{ATP}_{\text {PT }}$ directly. MitoK $\mathrm{K}_{\mathrm{ATP}}$ opening causes an influx in $\mathrm{K}^{+}\left(\uparrow \mathrm{K}^{+}\right)$leading to swelling $(\uparrow \Delta \mathrm{V})$ and alkalinization $(\uparrow p H)$ of the mitochondrial matrix. The increase in $\mathrm{pH}$ slightly inhibits the ETC at complex I and leads to superoxide formation, which is rapidly transformed into a signaling reactive oxygen species (ROS) in the matrix. This ROS activates a second pool of $\mathrm{PKC} \varepsilon$ on the inner face of the MIM (PKC $\varepsilon 2)$, blocking the mitochondrial permeability transition (MPT) and preventing cell death. 
cardioprotective signaling and that they arise due to mitoK $\mathrm{ATP}_{\text {Opening, the direct }}$ cause and location of the ROS production was unknown. Membrane potential $(\Delta \Psi)$ decreases upon influx of $\mathrm{K}^{+}$, but this effect is known to decrease ROS production and can be excluded. Swelling causes about $20 \%$ increase in matrix water; Andrukhiv et al. demonstrated that this has no effect on ROS production by lowering the osmolality of the assay medium to induce a $50 \%$ increase in mitochondrial matrix volume. Suspending mitochondria in a more acidic or alkaline medium, however, had significant effects, with a decrease in $\mathrm{pH}$ lowering ROS production and an increase in $\mathrm{pH}$ raising it. The researchers proposed that mitochondria produce ROS due to the alkalinization of the matrix upon potassium influx through mitoK $\mathrm{K}_{\text {ATP. }}$ They further separated the effects of extramitochondrial and matrix $\mathrm{pH}$, demonstrating that matrix alkalinization from $\mathrm{K}^{+}$influx is directly responsible for the increased ROS production (2).

Valinomycin, the $\mathrm{K}^{+}$-specific ionophore, also increases $\mathrm{ROS}$ production by the same amount as the KCOs when it is administered at a concentration that matches the $\mathrm{K}^{+}$-flux of the open mitoK $\mathrm{K}_{\text {ATP }}$ channel. This effect is not blocked by 5$\mathrm{HD}$, however, because valinomycin operates independently of mitoK $\mathrm{ATP}_{\mathrm{A}}$. At low concentrations, valinomycin exhibits a dose dependent increase in ROS production until that concentration that matches mitoK $\mathrm{KTP}_{\mathrm{AT}} \mathrm{K}^{+}$-flux. At any higher concentration ROS production is quickly diminished. This demonstrates that mitoK $\mathrm{K}_{\text {ATP }}$ opening has been finely tuned for the exact amount of $\mathrm{K}^{+}$-flux 
necessary to induce maximal ROS production. It can be inferred that mitoK $\mathrm{ATP}_{\mathrm{A}}$ opening is designed to produce ROS for secondary messaging (2).

ROS is generated in the form of superoxide $\left(\mathrm{O}_{2}{ }^{*}\right)$ when a single electron is transferred directly from a redox center in the electron transport system to molecular oxygen (141). "Superoxide of mitochondrial origin arise from three major sites (mechanisms): 1) the o-site of complex III, 2) redox centers in complex I that have been reduced by reverse electron transport from the Q-pool, and 3) redox centers in complex I that have been reduced by NADH" (Andrukhiv et al., 2006). Inhibitors of redox centers in the electron transport system can be used to identify the location of ROS formation. Myxothiazol inhibits the coenzyme Q-cytochrome c reductase complex and blocks ROS arising from complex III. It also stimulates ROS arising at complex I due to the downstream block on complex III and was indeed shown to cause a significant increase in ROS production, eliminating contribution

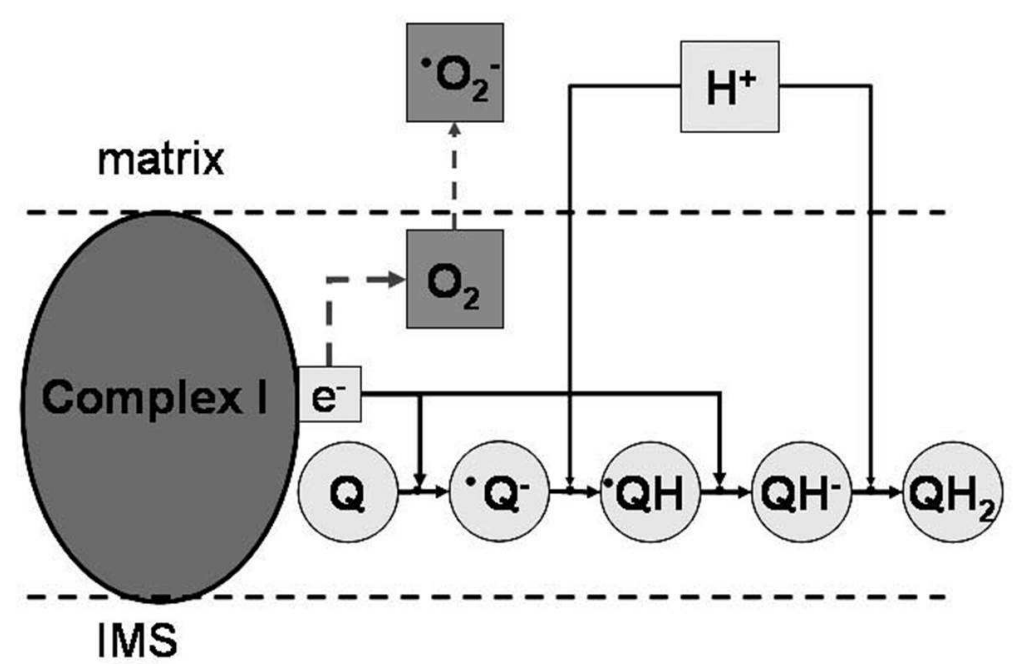

Fig. 1.6. Effect of matrix $\mathrm{pH}$ on ROS production from complex I. Electrons are passed to ubiquinone (Q) from complex I (NADH: ubiquinone oxidoreductase) of the electron transport chain. Although the site of superoxide production has been controversial, recent data suggest that the single electrons are delivered to oxygen from the $\mathrm{FMNH}_{2}$ or FMN semiquinone (13). It can be seen in the diagram that the reduction of ubiquinone requires two matrix protons. Electron flow will therefore be retarded at these points as matrix $\mathrm{pH}$ increases, causing increased reduction at the flavin site and consequent increase in steady-state superoxide production. IMS, intermembrane space. 
by mechanism 1 (2). Rotenone inhibits complex I, but it did not have any significant effect, suggesting that mechanism 2 is not a major contributor. ROS production by valinomycin was amplified by the addition of myxothiazol, suggesting that the ROS formed due to the increased $\mathrm{K}^{+}$influx is produced upstream of complex III. The positive effects of valinomycin and myxothiazol and the lack of effect of rotenone on ROS production point to mechanism 3 and superoxide production at complex I. The transfer of electrons to molecular oxygen to form superoxide is shown in Fig. 1.6.

\section{Mitochondrial ROS as second messengers}

Superoxide is quickly transformed by superoxide dismutase into hydrogen peroxide $\left(\mathrm{H}_{2} \mathrm{O}_{2}\right)$, which, is further transformed by enzymatic action or redox catalyzed reactions to other downstream ROS. The oxidation products of mitochondrial ROS act as second messengers in cardioprotective signaling and a host of other signaling processes. Mitochondrial ROS have a further role in triggering gene transcription at the nucleusand protein synthesis at the endoplasmic reticulum (46).

While these signaling ROS clearly play a crucial role in signaling throughout the cell and in numerous processes, the particular species operating in cardioprotective signaling has yet to be identified. The aim of this project is to accomplish this goal and determine the ROS acting as a second messenger in cardioprotective signaling. 


\title{
Chapter 2: Mitochondrial Reactive Oxygen Species - Which ROS Signals Cardioprotection?
}

\author{
Anders O. Garlid ${ }^{1}$, Martin Jaburek ${ }^{2}$, Jeremy P. Jacobs ${ }^{1}$, and Keith D. Garlid ${ }^{1 *}$ \\ ${ }^{1}$ Department of Biology, Portland State University, Portland, OR, 97201-0751, \\ 2Department of Membrane Transport Biophysics, Institute of Physiology, v.v.i., \\ Prague, Czech Republic, 14220
}

Running Head: Mitochondrial ROS Signaling

Keywords: mitochondria; cardioprotection; reactive oxygen species; K-ATP channels; cardiac ischemia; ROS signaling

Published with permission from American Journal of Physiology: Heart and Circular Physiology section (see Appendix A) 


\section{2-A: Abstract}

Mitochondria are the major effectors of cardioprotection by procedures that open the mitochondrial ATP-sensitive potassium channel (mitoK ATP), including ischemic and pharmacological preconditioning. MitoK $\mathrm{K}_{\mathrm{ATP}}$ opening leads to increased reactive oxygen species (ROS), which then activate a mitoK $\mathrm{ATP}_{\text {- }}$ associated $\mathrm{PKC} \varepsilon$, which phosphorylates mitoK $\mathrm{ATP}_{\mathrm{P}}$ and leaves it in a persistent open state (Costa, ADT and Garlid, KD. Am J Physiol 295, H874-82, 2008). The ROS responsible for this effect is not known. The present study focuses on superoxide $\left(\mathrm{O}_{2}{ }^{-}\right)$, hydrogen peroxide $\left(\mathrm{H}_{2} \mathrm{O}_{2}\right)$, and hydroxyl radical $\left(\mathrm{HO}^{\circ}\right)$, each of which has been proposed as the signaling ROS. Feedback activation of mitoK $\mathrm{KTP}_{\mathrm{A}}$ provides an ideal setting for studying endogenous ROS signaling. Respiring rat heart mitochondria were preincubated with ATP and diazoxide, together with an agent being tested for interference with this process, either by scavenging ROS or by blocking ROS transformations. The mitochondria were then assayed to determine whether or not the persistent phosphorylated open state was achieved. Dimethylsulfoxide (DMSO), dimethylformamide (DMF), deferoxamine, Trolox, and bromoenol lactone (BEL) each interfered with formation of the ROSdependent open state. Catalase, the $\mathrm{H}_{2} \mathrm{O}_{2}$ scavenger, did not interfere with this step. We also found that DMF blocked cardioprotection by both ischemic preconditioning and diazoxide. The lack of a catalase effect and the inhibitory effects of agents acting downstream of $\mathrm{HO}^{\circ}$ excludes $\mathrm{H}_{2} \mathrm{O}_{2}$ as the endogenous signaling ROS. Taken together, the results support the conclusion that the ROS 
message is carried by a downstream product of $\mathrm{HO}^{\circ}$ and that it is probably a product of phospholipid oxidation.

Keywords: mitochondria; cardioprotection; hydroxyl radical; K-ATP channels; mitochondrial permeability transition; cardiac ischemia; ROS signaling 


\section{2-B: Introduction}

Reactive oxygen species (ROS) are second messengers of preconditioning (50) and have long been known to be required for cardioprotective signaling $(6,15,40,103,135,153)$. The mechanism of increased ROS is reasonably well understood: signaling from the plasma membrane leads to opening of the mitochondrial ATP-sensitive $\mathrm{K}^{+}$channel (mitoK $\mathrm{ATP}_{\text {) }}(45)$, and the increased $\mathrm{K}^{+}$influx into the matrix causes an increase in ROS, which derive during normoxia from Complex I of the respiratory chain (2).

The ROS transformations that

take place in mitochondria are

summarized in Figs. 2.1 and 2.2.

The primary ROS produced by the

mitochondrial respiratory chain is

superoxide $\left(\mathrm{O}_{2}{ }^{-}\right)$, which is formed by

single-electron reduction of oxygen.

The majority of $\mathrm{O}_{2}{ }^{\cdot-}$ undergoes

dismutation to hydrogen peroxide

$\left(\mathrm{H}_{2} \mathrm{O}_{2}\right)$ both spontaneously and

through the action of superoxide

dismutases located in the matrix and

the intermembrane space $(12,75$,

101). Much of the $\mathrm{H}_{2} \mathrm{O}_{2}$ generated is

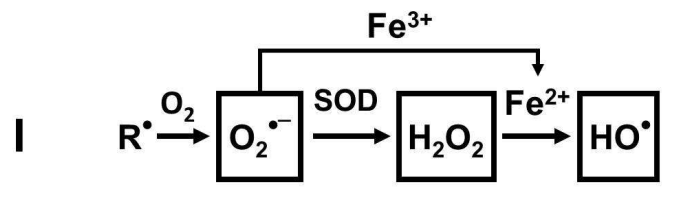

$$
\text { II } \mathrm{RH} \stackrel{\mathrm{HO}^{\circ}}{\longrightarrow} \mathrm{R}^{\cdot}+\mathrm{H}_{2} \mathrm{O} \stackrel{\mathrm{O}_{2}}{\rightarrow} \mathrm{ROO}^{\circ} \stackrel{\mathrm{RH}^{\mathrm{RH}}}{\rightarrow} \mathrm{ROOH}+\mathrm{R}^{\cdot}
$$

III $\mathrm{ROOH} \stackrel{\mathrm{Fe}^{2+}}{\longrightarrow} \mathrm{RO}^{\circ}+\mathrm{Fe}^{3+}+\mathrm{HO}^{-}$

alkoxyl radicals

Fig. 2.1. Mitochondrial electron transport chain-derived ROS and formation of alkyl peroxylalkoxyl radicals. (I) Superoxide $\left(\mathrm{O}_{2}{ }^{-}\right)$is formed by single electron transfer to molecular oxygen $\left(\mathrm{O}_{2}\right)$ from a reduced group $\left(\mathrm{R}^{\circ}\right)$ in the respiratory chain and converted to hydrogen peroxide $\left(\mathrm{H}_{2} \mathrm{O}_{2}\right)$ by superoxide dismutase (SOD). Hydroxyl radical $\left(\mathrm{HO}^{\circ}\right)$ is formed from $\mathrm{H}_{2} \mathrm{O}_{2}$ in the presence of a transition metal ion such as iron $\left(\mathrm{Fe}^{2+/ 3+}\right)$ (the Fenton reaction). (II) Subsequent reactions include the formation of alkyl peroxyl radicals $\left(\mathrm{ROO}^{\circ}\right)$ and alkyl hydroperoxides $(\mathrm{ROOH})$. $\mathrm{HO}^{\circ}$ acts on an alkyl side chain $(\mathrm{RH})$ to form a reduced group $\left(\mathrm{R}^{\circ}\right)$ that leads to the formation of ROO', and these react with $\mathrm{RH}$ to produce $\mathrm{ROOH}$. (III) Alkoxyl radicals $\left(\mathrm{RO}^{\circ}\right)$ are formed in the decomposition of hydroperoxides $(\mathrm{ROOH})$ by transition metal ions $\left(\mathrm{Fe}^{2+}\right)$. 
reduced by the glutathione and thioredoxin reductase antioxidant systems, which prevent the emission of excess $\mathrm{H}_{2} \mathrm{O}_{2}$ to the cytosol (132). $\mathrm{H}_{2} \mathrm{O}_{2}$ is also decomposed by catalase, which is present in the matrix of heart mitochondria (121). Some of the $\mathrm{H}_{2} \mathrm{O}_{2}$ leads to formation of hydroxyl radical ( $\left.\mathrm{HO}^{\circ}\right)$ through the Fenton reaction of $\mathrm{H}_{2} \mathrm{O}_{2}$ with transition metal ions (83). The highly reactive $\mathrm{HO}$ ' oxidizes proteins and lipids in diffusion limited reactions (78).

The consequences of ROS signaling for mitochondrial physiology are that two mitochondrial protein kinase $\mathrm{C}$ epsilons $(\mathrm{PKC} \varepsilon$ ) are activated by oxidation of their thiol groups (82). Activation of PKCE1, which is associated with mitoK $\mathrm{K}_{\mathrm{ATP}}$ at the mitochondrial inner membrane, leads to opening of mitoK $\operatorname{ATP}(20,71)$.

Activation of PKCE2 leads to inhibition of the mitochondrial permeability transition

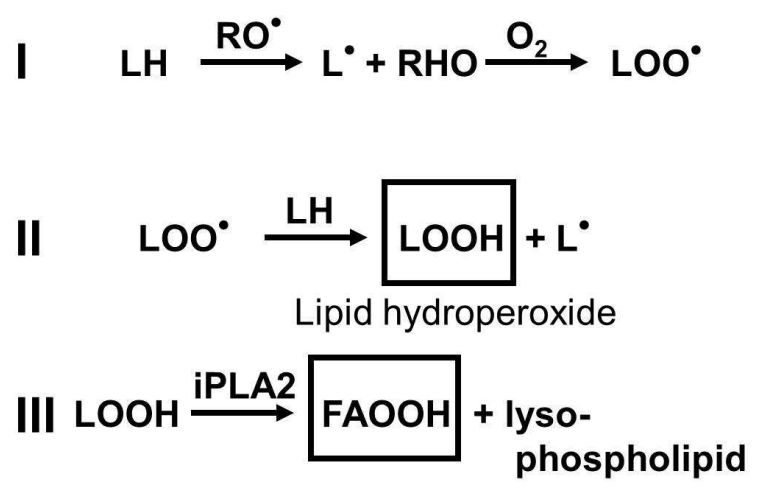

Fatty acid hydroperoxide

Fig. 2.2. Lipid peroxidation and release of hydroperoxy fatty acids.(I and II)alkoxyl (RO'), hydroxyl (RHO), and alkyl peroxyl radicals (LOO') can initiate the non-enzymatic chain reaction leading to lipid peroxidation $(\mathrm{LOOH})$. (III) The lipid hydroperoxides (LOOH) can be released by the action of mitochondrial phospholipases, such as $\mathrm{Ca}^{2+}$-independent phospholipases PLA2 (iPLA2), which have been found to modulate the function of mitochondria (see Cedars et al (13) for a review). The action by iPLA2 results in a hydroperoxy fatty acid (FAOOH).
(MPT) (18). Progress is being made in these areas and in the molecular identification of mitoK $\mathrm{K}_{\mathrm{ATP}}$ (34). However, the ROS responsible for activating these PKCEs in vitro or in vivo is still not known. Because the signaling ROS not only originates in mitochondria but also acts on mitochondria, it should be possible to narrow the search for the signaling ROS by studies on isolated rat heart 
mitochondria. The objective of these studies was to identify the point in the reaction sequence of ROS transformations at which the ROS signal is formed and, ultimately, to determine the identity of the ROS signal itself.

We previously described feedback activation of mitoK $\mathrm{KTTP}_{\mathrm{A}}$, in which mitoK $\mathrm{K}_{\text {ATP }}$ opening by a $\mathrm{K}_{\mathrm{ATP}}$ channel opener leads to endogenous $\mathrm{ROS}$ formation and a persistent open state of mitoK $\mathrm{K}_{\mathrm{ATP}}$. The phenomenon was shown to involve PKC\&1 and an inner membrane phosphorylation event (20). This persistent open state of mitoK $\mathrm{K}_{\text {ATP }}$ is thought to be responsible for "memory," which is seen with all preconditioning (20).

We employed a preincubation protocol to probe the feedback activation system for the identity of the signaling ROS. Diazoxide was used to induce mitoK $\mathrm{K}_{\text {ATP }}$ opening, which leads to endogenous ROS formation and activation of PKCع1. This process establishes the prolonged phosphorylation-dependent open state of mitoK $\mathrm{K}_{\mathrm{ATP}}(20)$. We then re-isolated the mitochondria and probed them for this state by measuring the resulting steady-state mitochondrial matrix volume using the light scattering technique $(9,17,21,47)$. Agents or conditions that interfered with any step in the generation of the signaling ROS would prevent formation of the phosphorylation dependent open state. Two additional assays were carried out to eliminate agents that interfered either with mitoK $\mathrm{ATP}$ dependent $\mathrm{K}^{+}$uptake or with ROS-activation of PKC\&1. Together, these procedures allowed identification of agents that specifically interfered with formation of the ROS signal. 
The agents that were found to block formation of the ROS signal were dimethylsulfoxide (DMSO) and dimethylformamide (DMF), scavengers of $\mathrm{HO}$ and $\mathrm{RO}^{\circ}(5,99)$, trolox, a chain-breaking antioxidant and peroxyl radical scavenger (95), deferoxamine, an iron chelator $(83,131)$, and bromoenol lactone (BEL), which is an inhibitor of $\mathrm{Ca}^{2+}$-independent phospholipase A2 (iPLA2) (13). Based on the site of action of these agents, we conclude that the ROS signal arises downstream of $\mathrm{HO}^{\circ}$ formation. Because $\mathrm{H}_{2} \mathrm{O}_{2}$ is produced upstream of $\mathrm{HO}^{*}$, these results exclude $\mathrm{H}_{2} \mathrm{O}_{2}$ as the signaling ROS. This conclusion is supported by the finding that catalase did not interfere with formation of the phosphorylation-dependent open state of mitoK $\mathrm{K}_{\text {ATP. }}$.

We performed experiments on MPT, in which diazoxide was used to induce inhibition of MPT via ROS activation of PKC\&2 (18). This effect was blocked by DMSO, also implicating a ROS signal downstream from $\mathrm{HO}^{*}$. Finally, we found that the $\mathrm{HO}^{\circ}$ scavenger DMF blocked cardioprotection by ischemic preconditioning and diazoxide. Taken together, each of these results supports the conclusion that the ROS message is carried by a downstream product of hydroxyl radical $\left(\mathrm{HO}^{\circ}\right)$. The actions of $\mathrm{BEL}$ suggest a product of phospholipid oxidation. 


\section{2-C: Research Design and Methods}

\section{Langendorff perfused hearts}

Hearts from male Sprague-Dawley rats (200-240g) were perfused as previously described $(28,51,115,120)$ with Krebs Henseleit buffer containing (in mM) $118 \mathrm{NaCl}, 5.9 \mathrm{KCl}, 1.75 \mathrm{CaCl}_{2}, 1.2 \mathrm{MgSO}_{4}, 0.5 \mathrm{EDTA}, 25 \mathrm{NaHCO}_{3}, 16.7$ glucose at $37^{\circ} \mathrm{C}$ and $\mathrm{pH} 7.4$ and gassed with $95 \% \mathrm{O}_{2}-5 \% \mathrm{CO}_{2}$. Treatment protocols are described in Fig. 2.3. Hearts were stabilized for 25 min with Krebs Henseleit buffer prior to treatment with buffer containing drugs or agents then subjected to 25 minutes global ischemia followed by 2 hours of reperfusion and measurement of infarct size. Ischemic

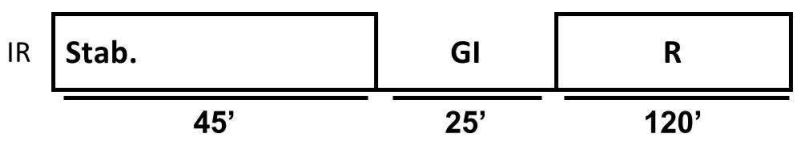

preconditioning (IPC) was

established by two cycles of $5 \mathrm{~min}$ global ischemia followed by $5 \mathrm{~min}$ reperfusion prior to the index

ischemia. Ischemic

postconditioning was performed

with 6 cycles of $10 \mathrm{~s}$ ischemia plus $10 \mathrm{sec}$ reperfusion (140). Hearts were not paced and mechanical performance was evaluated as the

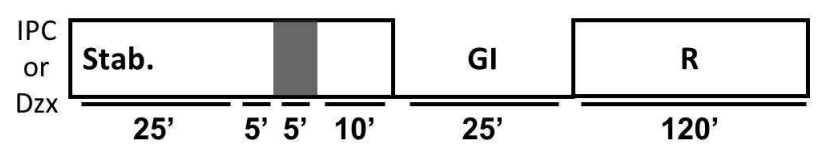

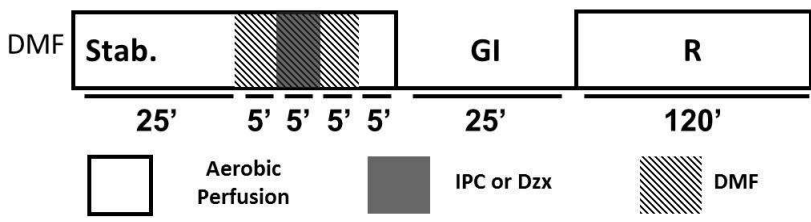

Fig. 2.3. Isolated heart protocols. Hearts were perfused with buffer, as described in "Methods." After 45 min of stabilization, hearts were subjected to 25 minutes global ischemia ("Gl"), followed by 2 hours of reperfusion ("R") and, finally, processing for infarct size estimation. The protocol for ischemic reperfusion without additional treatment is labeled "IR." Ischemic preconditioning ("IPC") was established by $5 \mathrm{~min}$ global ischemia followed by 10 min reperfusion prior to the index ischemia. Diazoxide ("Dzx") $(50 \mu \mathrm{M})$ was perfused for $5 \mathrm{~min}$, followed by 10 min reperfusion with buffer prior to the index ischemia. Dimethylformamide ("DMF") (1\% v/v) was administered $5 \mathrm{~min}$ before the first IPC ischemia and before diazoxide; it was continued for 5 min after these treatments and this was followed by $5 \mathrm{~min}$ reperfusion with buffer prior to the index ischemia. 
product of heart rate and left ventricular developed pressure (LVDP) and reported as a percentage of rate pressure product at stabilization, $t=0$ (RPP $\%$ at $t=0$ ). Infarct size (IS) was determined by the method of Ytrehus et al. (152) whereby the treated hearts were stained with triphenyltetrazolium chloride (TTC) after reperfusion, fixed in formaldehyde and sliced into thin sections. Infarct size is reported as a percentage of the total cross-sectional area of the heart slices at risk. Experimental protocols complied with the Guiding Principles in the Use and Care of Animals published by the National Institutes of Health and were approved by IACUC at Portland State University.

\section{Mitochondrial isolation}

Male Sprague-Dawley rats $(200-240 \mathrm{~g})$ were anesthetized with $\mathrm{CO}_{2}$, immediately decapitated, and the hearts were removed for mitochondrial isolation exactly as described previously (21). Hearts were washed and finely minced in ice-cold isolation buffer containing $250 \mathrm{mM}$ sucrose, $10 \mathrm{mM}$ HEPES at $\mathrm{pH} 7.2$ and $0.5 \mathrm{mM} \mathrm{K}^{+}$-EGTA. The suspension was diluted 3-fold with isolation buffer supplemented with $1 \%$ fatty acid-free BSA. The time between decapitation and completion of homogenization was kept as brief as possible and was completed within 2 min; mitoK $\mathrm{K}_{\text {ATP }}$ activity shows a sharp dependence on the length of this period. The suspension was homogenized with a motorized Teflon pestle and centrifuged for $3 \mathrm{~min}$ at $1,500 \mathrm{~g}$. The supernatant was centrifuged for $5 \mathrm{~min}$ at $9,000 \mathrm{~g}$, and the resulting pellets were resuspended in isolation buffer lacking BSA and centrifuged for $3 \mathrm{~min}$ at 2,300 $\mathrm{g}$. This supernatant was centrifuged for 5 
min at 9,000 $\mathrm{g}$. The final mitochondrial pellet was resuspended with isolation buffer to $35-40 \mathrm{mg}$ protein/ $\mathrm{ml}$ and kept on ice. Where indicated, rat liver mitochondria were isolated using a similar protocol, as previously described (9). Mitochondrial protein concentration was estimated using the Biuret reaction (54).

Measurements of mitoK $K_{A T P}$ activity

MitoK $_{\text {ATP }}$ opening causes mitochondrial swelling due to respiration-driven uptake of $\mathrm{K}^{+}$salts and water. These volume changes were followed by light scattering, as previously described $(9,17,21,47)$. Light scattering changes of $0.1 \mathrm{mg} / \mathrm{ml}$ mitochondrial suspensions were followed at $520 \mathrm{~nm}$ and $30^{\circ} \mathrm{C}$. Mitochondria were suspended in a buffered salt assay medium containing $\mathrm{K}^{+}$ salts of $\mathrm{Cl}^{-}(120 \mathrm{mM})$, HEPES $(10 \mathrm{mM})$, EGTA $(0.1 \mathrm{mM})$, succinate $(10 \mathrm{mM})$, $\mathrm{MgCl}_{2}(0.5 \mathrm{mM})$, phosphate $(5 \mathrm{mM})$, ATP $(200 \mu \mathrm{M})$, rotenone $(2.5 \mu \mathrm{M})$, and oligomycin $(1 \mu \mathrm{g} / \mathrm{ml})$ at $\mathrm{pH} 7.2$. Osmolality ranged between 275 to $280 \mathrm{mOsm}$.

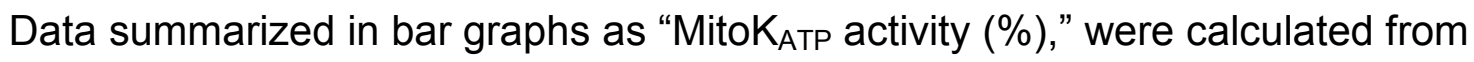

$$
100 \times \frac{A^{-1}(x)-A^{-1}(A T P)}{A^{-1}(0)-A^{-1}(A T P)}
$$

where $A^{-1}(x)$ is the observed inverse absorbance at $120 \mathrm{~s}$ under the given experimental condition, and $A^{-1}(A T P)$ and $A^{-1}(0)$ are values independently measured in the presence and absence of ATP, respectively.

\section{Preincubation protocol}

A preincubation protocol was used to trigger activation of PKC $\varepsilon$ by endogenous ROS. As illustrated in Fig. 2.4, mitochondria were incubated in the 
light scattering assay medium

(described above) for 3 minutes at

$30^{\circ} \mathrm{C}$ with $30 \mu \mathrm{M}$ diazoxide (Dzx). The

preincubation medium was

supplemented with various agents to

explore the mechanisms of

endogenous ROS signaling. Each

sample was equally divided and

preincubated in the presence or

absence of diazoxide. The treated

mitochondria were then isolated from

the incubation medium and

resuspended in a small volume (15

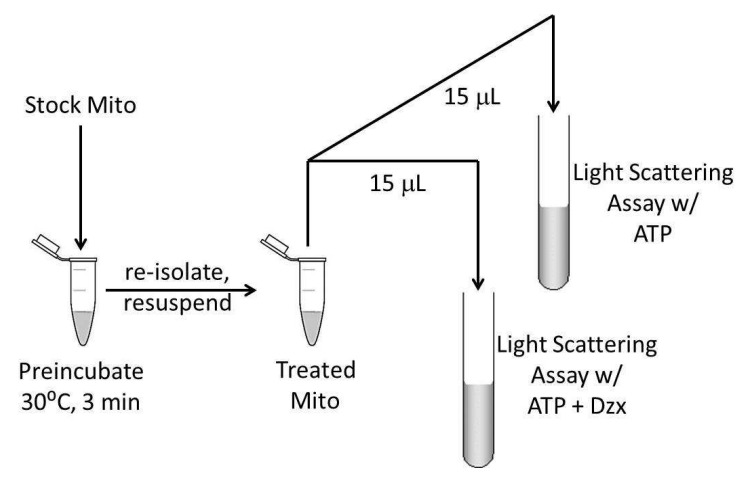

Fig. 2.4. Preincubation protocol. Isolated rat heart mitochondria suspended in $250 \mathrm{mM}$ sucrose and $10 \mathrm{mM} \mathrm{HEPES}$ at $30 \mathrm{mg} / \mathrm{mL}$ ("Stock Mito") was incubated at $30^{\circ} \mathrm{C}$ for 3 minutes in assay medium containing $200 \mu \mathrm{M}$ ATP and $30 \mu \mathrm{M}$ diazoxide (Dzx), in a total volume of $0.5 \mathrm{~mL}$. The treated mitochondria were reisolated and resuspended, and mitoK $_{\text {ATP }}$ activity was measured using the light scattering assay, with a final protein concentration of $0.1 \mathrm{mg} / \mathrm{mL}$. Half of the treated mitochondria were assayed with ATP and the other half with ATP and Dzx. The incubation was supplemented with various agents including 5-HD $(300 \mu \mathrm{M}), \varepsilon \mathrm{V}_{1-2}(0.5 \mu \mathrm{M})$, catalase $(250 \mathrm{U} / \mathrm{mL})$, DMSO $(1 \% \mathrm{v} / \mathrm{v})$, DMF $(1 \% \mathrm{v} / \mathrm{v})$, Trolox $(100 \mu \mathrm{M})$, deferoxamine $(1$ $\mathrm{mM})$, MPG $(1 \mathrm{mM})$, or BEL $(10 \mu \mathrm{M})$.

$\mu \mathrm{l})$ of ice cold sucrose buffer containing $250 \mathrm{mM}$ sucrose and $10 \mathrm{mM}$ HEPES at

$\mathrm{pH}$ 7.2. MitoK $\mathrm{K}_{\mathrm{ATP}}$ activity of the treated mitochondria was then assayed using light scattering. When preincubated without diazoxide, the mitochondria behaved normally in the subsequent assay. That is, mitoK $\mathrm{K}_{\text {ATP }}$ activity was inhibited by ATP, and inhibition was released by diazoxide. When preincubated with diazoxide, mitoK $\mathrm{K}_{\text {ATP }}$ activity in the subsequent assay was not inhibited by ATP, and diazoxide had no effect. Thus, these conditions cause mitoK $\mathrm{K}_{\mathrm{ATP}}$ to remain open due to its phosphorylation by $\mathrm{PKC} \varepsilon(20)$. Any agent that blocks any step in the process will block feedback activation of mitoK $\mathrm{K}_{\text {ATP. }}$. 
Measurements of mitochondrial permeability transition

Mitochondrial permeability transition (MPT) activity was monitored by light scattering in medium containing $200 \mu \mathrm{M}$ ATP and lacking EGTA. MPT opening was synchronized by sequential additions of $\mathrm{CaCl}_{2}\left(100 \mu \mathrm{M}\right.$ free $\left.\mathrm{Ca}^{2+}\right)$, ruthenium red $\left(0.1 \mu \mathrm{M}\right.$, to block further $\mathrm{Ca}^{2+}$ uptake), and carbonyl cyanide $\mathrm{m}$ chlorophenyl hydrazine (CCCP) (250 nM), at 20, 40 and $60 \mathrm{sec}$, respectively (18). These experiments used mitochondria from livers taken from the animals supplying the heart.

\section{Statistical analysis}

Data are presented as mean \pm standard deviation (SD) of the mean. Oneway ANOVA followed by Tukey's post hoc test or Student's t-test of the means were used as required to analyze the data using Microcal Origin software (Northampton, MA, USA). A value of $p<0.05$ was considered statistically significant.

\section{Chemicals}

Peroxynitrite was acquired from Cayman Chemical. All other chemicals were obtained from Sigma Chemical Co. (St. Louis, MO, USA). N-2mercaptopropionylglycine (MPG) was always freshly prepared and adjusted to neutral $\mathrm{pH}$ by Tris base. Diazoxide and $\varepsilon \mathrm{V}_{1-2}$ were prepared in DMSO and bromoenol lactone (BEL) was always freshly prepared in DMSO. Uric acid, 
trolox, deferoxamine, catalase, 5-hydroxydecanoate (5-HD) and ruthenium red were prepared in distilled water. 


\section{2-D: Results}

Endogenous ROS signaling and PKCE1-dependent feedback activation of mitoK $_{\text {ATP. }}$ Feedback activation of mitoK $\mathrm{K}_{\mathrm{ATP}}$ can be divided into three steps, described in Fig. 2.5. In Step 1, $\mathrm{K}^{+}$ channel openers such as diazoxide open mitoK $\mathrm{ATP}(50)$, and the

resulting $\mathrm{K}^{+}$influx and matrix

alkalinization leads to increased

generation of superoxide (2). Step

2encompasses the ROS

transformations that result in

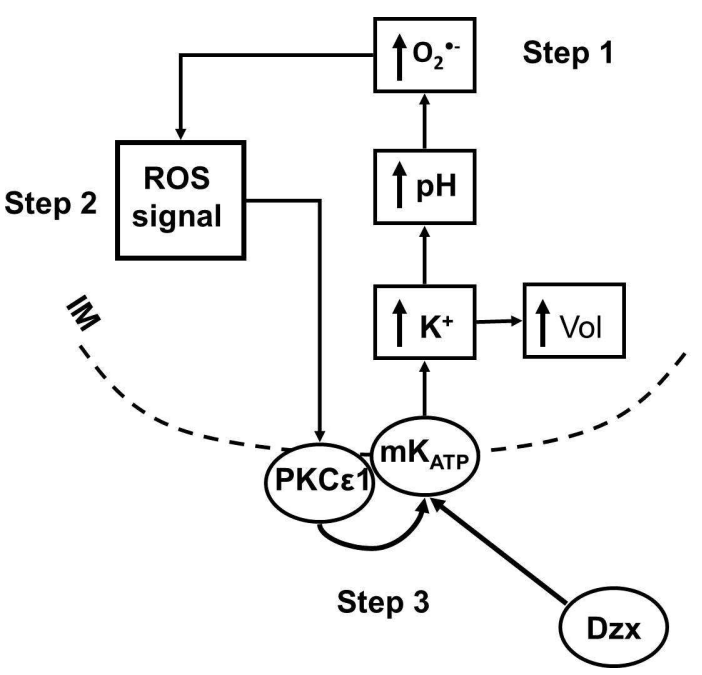

formation of the signaling ROS, i.e., the ROS that activates PKCE1. In

Step 3, the activated PKC\&1

establishes the phosphorylation

dependent open state of mitoK $\mathrm{K}_{\mathrm{ATP}}$

(20). The rationale for this division

is that we can study the effects of

agents on the overall cycle and,

independently, on steps 1 and 3 . In

this way, we can identify agents

that interfere specifically with

Fig. 2.5. ROS-dependent feedback activation of mito $K_{A T P}$. Step 1 of the process encompasses the sequence from mitochondrial ATP-sensitive $\mathrm{K}^{+}$channel ("mitoK $\mathrm{ATP}_{\mathrm{P}}$ ") opening to superoxide $\left(\mathrm{O}_{2}{ }^{-}\right)$formation. MitoK $\mathrm{K}_{\text {ATP }}$ is opened by $\mathrm{K}_{\text {ATP }}$ channel openers such as diazoxide ("Dzx") (50). The consequent uptake of $\mathrm{K}^{+}$and anions leads to increased matrix volume (" $\uparrow$ Vol"), which is the basis of the light scattering assay for mitoK $\mathrm{K}_{\text {ATP }}$ activity (21). The cytosolic concentration difference between $\left[\mathrm{K}^{+}\right]$and [phosphate] means that more $\mathrm{K}^{+}$than phosphate will be taken up, leading to matrix alkalinization (“ $\uparrow \mathrm{pH}$ ”) (21). Matrix alkalinization, in turn, inhibits Complex I, leading to increased production of superoxide (" $\uparrow \mathrm{O}_{2}{ }^{\cdot-")}$ (2). Step 2encompasses the many ROS transformations that take place and lead to the signaling ROS, which activates protein kinase C-epsilon 1 (PKC\&1). In Step 3, the activated $\mathrm{PKC} \varepsilon 1$ phosphorylates a protein, possibly mitoK $\mathrm{K}_{\text {ATP }}$ itself, which leads to the phosphorylated open state of $\operatorname{mitoK}_{\text {ATP }}(20)$. In parallel (not represented here), the ROS signal activates PKC 2 , leading to inhibition of MPT opening (18). 
formation of the signaling ROS (Step 2).

Fig. 2.6 contains results of control experiments using the preincubation protocol described in "Methods." When mitochondria were preincubated with diazoxide and ATP, they exhibited maximum volume change in the normal assay medium containing ATP and no further effect was seen with the addition of

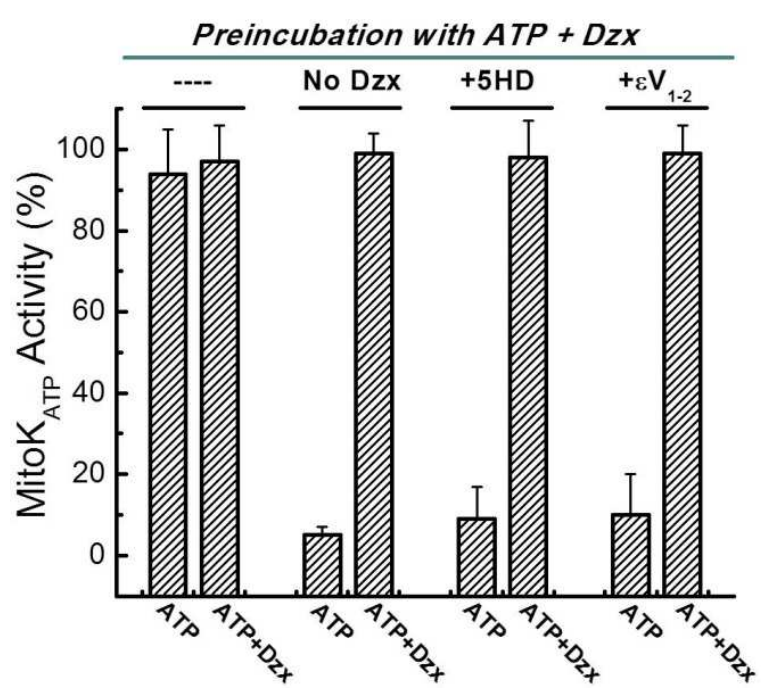

Fig. 2.6. Feedback activation of mito $K_{A T P}$ is blocked by 5-hydroxydecanoate (5-HD) and $\varepsilon V_{1-2}$. Shown are the effects of various agents on mitoK $\mathrm{K}_{\mathrm{ATP}}$ activity after preincubation with ATP $(200 \mu \mathrm{M})$ plus diazoxide (Dzx) $(30 \mu \mathrm{M})$. Mitochondria were preincubated with the agents indicated at the top of the figure, then assayed in $\mathrm{K}^{+}$medium with ATP or ATP + Dzx as indicated below (described in "Methods"). With no further additions to the preincubation ("----"), mitoK ATP remains in the phosphorylated open state with full activity, and diazoxide ("ATP+Dzx") has no further effect in the subsequent light scattering assay. When mitoK $\mathrm{K}_{\mathrm{ATP}}$ opening during preincubation was prevented by omission of diazoxide ("No Dzx") or inclusion of the mitoK $\mathrm{K}_{\mathrm{ATP}}$ blocker 5-HD $(300 \mu \mathrm{M})(“+5-\mathrm{HD}$ "), the phosphorylation-dependent open state was blocked. When feedback activation of PKC 11 was prevented by inclusion of $\varepsilon \bigvee_{1-2}(0.5 \mu \mathrm{M})$ $\left("+\varepsilon \bigvee_{1-2}\right.$ "), the open state was also blocked. Data are means $\pm S D$ of at least 3 independent experiments. ${ }^{*} p<0.05$. diazoxide (first two bars of Fig. 2.6).

This result is characteristic of

conditions that allow mitoK $\mathrm{K}_{\text {ATP }}$ to

achieve the phosphorylation-

dependent open state. When Step 1

was blocked by omission of

diazoxide (second pair of bars) or

inclusion of 5-hydroxydecanoate (5-

HD) (third pair of bars) in the

preincubation, mitoK $\mathrm{K}_{\mathrm{ATP}}$ behaved as

if there were no preincubation; that

is, mitoK $\mathrm{K}_{\text {ATP }}$ activity was inhibited by

ATP and opened by diazoxide.

When Step 3 was blocked by

inclusion of the specific PKCE

inhibitor $\varepsilon \bigvee_{1-2}$ in the preincubation

(last pair of bars), feedback 
activation was also prevented and

mitoK $_{\text {ATP }}$ responded normally to ATP and diazoxide.

Fig. 2.7 contains results of preincubation experiments in which we evaluated agents that may block ROS signaling (Step 2) in the feedback loop shown in Fig. 2.5. Dimethylsulfoxide (DMSO), and dimethylformamide (DMF), and N-2mercaptoprionylglycine (MPG) have all been described as reactants with $\mathrm{HO}^{\circ}, \mathrm{RO}^{\circ}$ (alkoxyl radicals), and ROO` (alkylperoxyl radicals) $(5,99)$, each of which may play a role in endogenous ROS signaling. Trolox, a vitamin $E$ analogue, acts as a chainbreaking antioxidant and peroxyl radical scavenger (95). Fig. 2.7A demonstrates that each of these agents prevented formation of the phosphorylation-dependent open
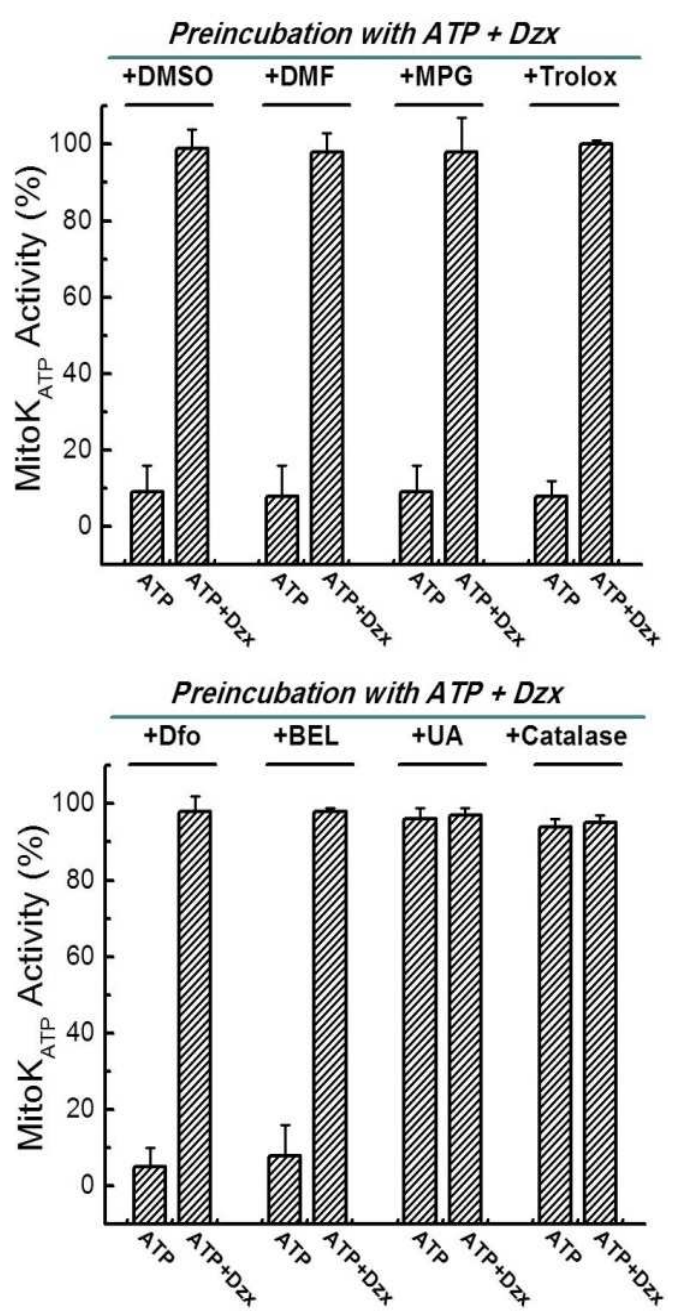

Fig. 2.7. Effects of agents that may block formation of the signaling ROS on feedback activation of mito $K_{\text {ATP. }}$. Shown in (A) are the effects of various ROS and radical scavenging agents and antioxidants on mitoK $\mathrm{K}_{\text {ATP }}$ activity after preincubation with ATP plus diazoxide (Dzx) in the presence of dimethylsulfoxide (DMSO) $(1 \% \mathrm{v} / \mathrm{v})$, dimethylformamide (DMF) (1\%v/v), N-2-mercaptopropionylglycine (MPG) $(1 \mathrm{mM})$, and Trolox $(100 \mu \mathrm{M})$. Each of these agents blocked formation of the phosphorylation-dependent open state of mitoK $_{\text {ATP. }} \ln (B)$, deferoxamine (Dfo) $(1 \mathrm{mM})$ and bromoenol lactone (BEL) $(10 \mu \mathrm{M})$ also blocked this state, but uric acid $(100 \mu \mathrm{M})$ ("+UA") (peroxynitrite scavenger) and catalase $(250 \mathrm{U} / \mathrm{mL})\left(\mathrm{H}_{2} \mathrm{O}_{2}\right.$ scavenger) did not interfere with feedback activation of mitoK $\mathrm{K}_{\text {ATP. }}$. Mitochondria were preincubated with the agents indicated in the figure, then assayed in $\mathrm{K}^{+}$medium, as described in "Methods." Data are means of mitoK $K_{\text {ATP }}$ activity $\pm S D$ of at least 3 independent experiments. ${ }^{*} p<0.05$. 
state, suggesting that $\mathrm{HO}^{\circ}$ is involved in the generation of the signaling ROS.

Deferoxamine is a chelator of iron $(83,131)$ and acts as a preventive antioxidant by reducing the rate of iron-dependent hydroxyl and peroxyl radical chain initiation. $\mathrm{BEL}$ (racemic bromoenol lactone) is an inhibitor of $\mathrm{Ca}^{2+}-$ independent phospholipase (iPLA2) (13) and it prevents the release of oxidized phospholipids such as hydroperoxy fatty acids $(\mathrm{FAOOH})$ from the bilayer, a possible downstream oxidation product in the reaction sequence. Fig. 2.7B shows that both deferoxamine and BEL blocked feedback activation of mitoK $\mathrm{K}_{\mathrm{ATP}}$, further suggesting that hydroxyl radicals and their downstream oxidation products are involved in the generation of the signaling ROS. When uric acid (UA), a peroxynitrite scavenger (137), was included in the preincubation, the phosphorylation-dependent open state was preserved, indicating that peroxynitriteis not involved with any of the steps and, therefore, is not the signaling ROS. Catalase is an enzyme that catalyzes the decomposition of $\mathrm{H}_{2} \mathrm{O}_{2}$ and is present in the matrix of heart mitochondria (121). However, catalase was also unable to block the process of achieving the phosphorylated open-state, suggesting that $\mathrm{H}_{2} \mathrm{O}_{2}$ is not the signaling ROS.

The mechanisms of action of these agents suggests that they are acting on Step 2, the formation of the ROS signal; however it is necessary to demonstrate that they are not acting elsewhere in the loop, i.e. on Step 1 or Step 3. Accordingly, we tested each of the agents for their effect on mitoK $\mathrm{K}_{\text {ATP }}$ activity in the straight light scattering assay with no preincubation and found that none of

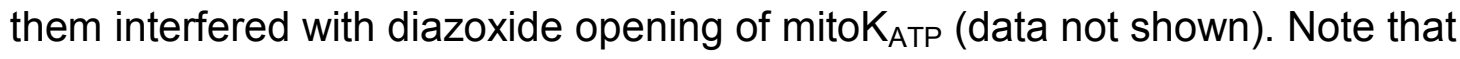


this assay also detects respiratory inhibition or uncoupling, which inhibit $\mathrm{K}^{+}$ uptake by reducing the driving force.

To evaluate their effect on Step 3 (induction of the phosphorylated open state by PKCE1), we examined whether the agents were able to block mitoK $\mathrm{ATP}_{\mathrm{P}}$ opening induced by $\mathrm{H}_{2} \mathrm{O}_{2}$, which works by activating PKC\&1 (20). Fig. 2.8 contains representative light scattering traces using mitochondria without preincubation. Here, we find that Trolox had no effect. MPG did block $\mathrm{H}_{2} \mathrm{O}_{2-}$ induced mitoK $\mathrm{ATP}_{\mathrm{T}}$ opening, which means that we cannot distinguish whether MPG acts on Step 2 or Step 3 from these experiments. The results of the Step 3 assays are summarized in Fig. 2.9 and show that deferoxamine, DMSO, DMF,

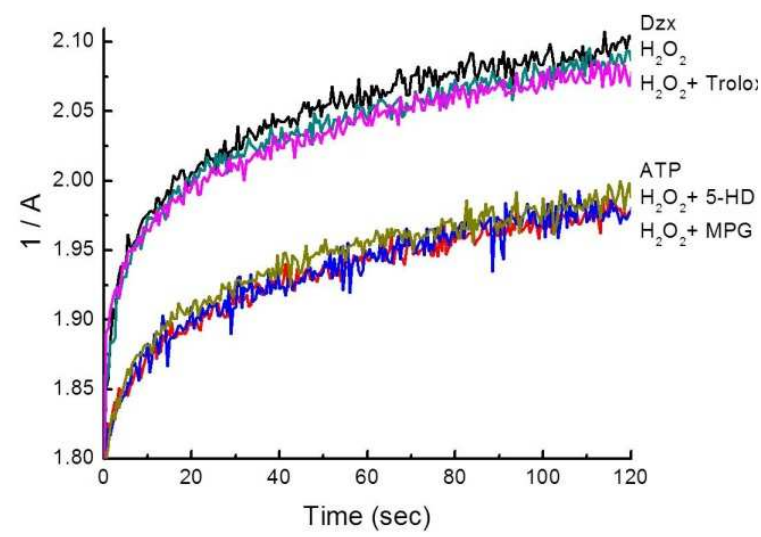

Fig. 2.8. Differential effects of MPG and Trolox on $\mathrm{H}_{2} \mathrm{O}_{2}$-dependent mitoK $K_{A T P}$ opening. Shown are representative light scattering traces (1/A vs. time) of rat heart mitochondria respiring on succinate in $\mathrm{K}^{+}$medium. Mitochondria were suspended at $0.1 \mathrm{mg} / \mathrm{ml}$ and assayed as described in "Methods." ATP $(200 \mu \mathrm{M})$ was present in all experiments. Where indicated, Trolox, MPG, 5-HD or no additions ("ATP") were present in the assay medium prior to the addition of mitochondria. Mitochondrial swelling was initiated by the addition of hydrogen peroxide $\left(\mathrm{H}_{2} \mathrm{O}_{2}\right)(2 \mu \mathrm{M})$ or diazoxide 2 seconds after the addition of mitochondria to trigger PKC\&1-dependent mitoK $_{\text {ATP }}$ opening.

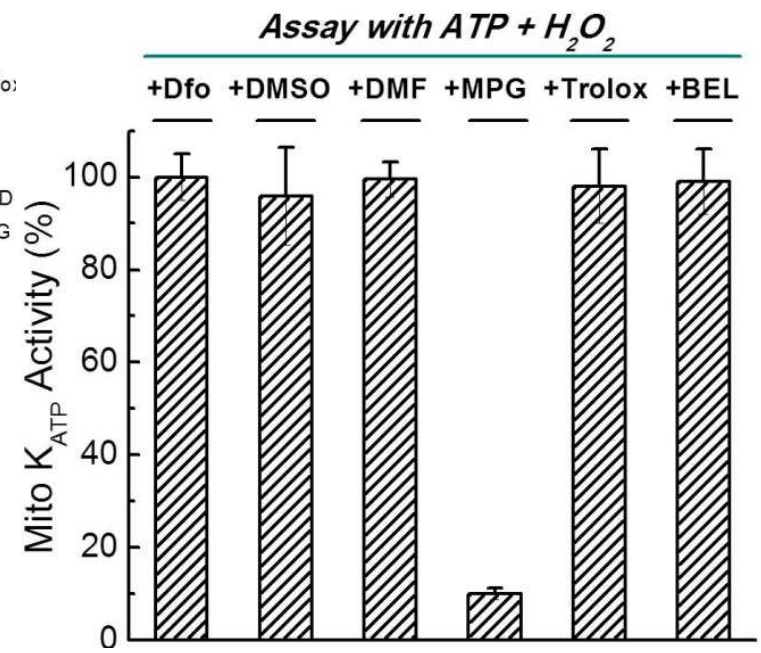

Fig. 2.9. Effects of agents on activation of PKC 1 by $\mathrm{H}_{2} \mathrm{O}_{2}$. The agents that were utilized in the preincubation experiments of Fig. 2.6 were examined for their effects on $\mathrm{H}_{2} \mathrm{O}_{2}{ }^{-}$ induced $\mathrm{PKC} \varepsilon$-dependent mitoK $\mathrm{K}_{\mathrm{ATP}}$ opening in the straight light scattering assay described in "Methods." MPG was the only agent that interfered with this step in the process. Deferoxamine, DMSO, DMF, Trolox, and BEL had no effect on $\mathrm{H}_{2} \mathrm{O}_{2}$ activation. Data are means \pm SD of at least 3 independent experiments. 
Trolox, and BEL did not inhibit $\mathrm{H}_{2} \mathrm{O}_{2-}$

induced mitoK $\mathrm{ATP}_{\mathrm{AT}}$ opening (Step 3).

Because they also did not interfere

with diazoxide opening of mitoK $\mathrm{K}_{\mathrm{ATP}}$

(Step 1), we infer that these agents

are acting to prevent formation of the

endogenous ROS signal that

activates PKCE1 (Step 2).

These findings may be

summarized as follows. 1)

Deferoxamine, DMSO, DMF, Trolox,

and BEL each prevent formation of

the endogenous ROS signal. These

results imply that the ROS signal

arises downstream from $\mathrm{HO}^{\circ}$ radical.

2) $\mathrm{H}_{2} \mathrm{O}_{2}$ is produced upstream of $\mathrm{HO}^{\circ}$

radical, and catalase, the $\mathrm{H}_{2} \mathrm{O}_{2}$
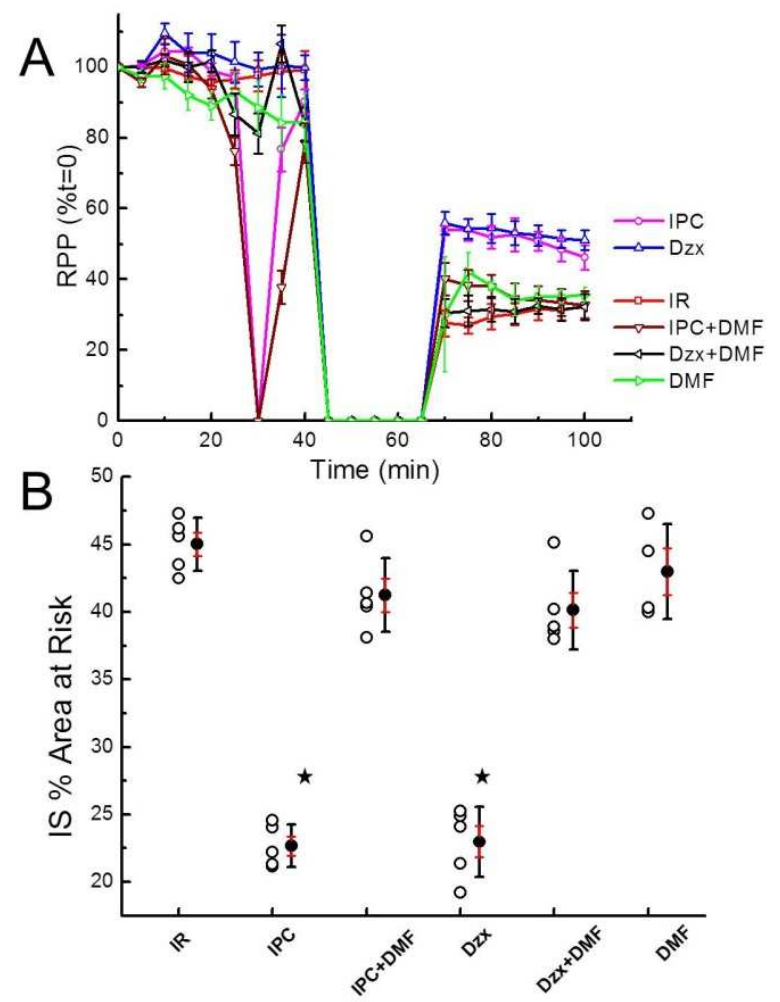

Fig. 2.10. Dimethylformamide (DMF) blocks protection of the perfused heart by diazoxide and IPC.(A) Shown are measurements of ratepressure product ("RPP ( $\%$ at $t=0)$ ") with time from perfusion of the ex vivo heart. Diazoxide $(50 \mu \mathrm{M})$ ("Dzx") and ischemic preconditioning ("IPC") improved functional recovery, and this effect was blocked by dimethylformamide ("Dzx + DMF" and "IPC + DMF"), a free radical scavenger. DMF alone had no effect. (B)Infarct size as a percent of area at risk ("IS \% Area at Risk") is plotted for the various treatments. Treatment with diazoxide (Dzx) and IPC reduced infarct size, and this effect was blocked by DMF. Data are means \pm SD of at least 3 independent experiments. ${ }^{*} p<0.05$.

scavenger, had no effect on the

formation of the phosphorylation-dependent open state. Therefore, $\mathrm{H}_{2} \mathrm{O}_{2}$ can be excluded as the signaling ROS. 3) The action of MPG is independent of ROS scavenging. Like all thiols, MPG can scavenge $\mathrm{HO}^{\circ}$, but $\mathrm{HO}^{\circ}$ is not involved in the pathway responsible for the results in Fig. 2.8. Moreover, MPG has little or no $\mathrm{H}_{2} \mathrm{O}_{2}$ scavenging ability $(10,91)$. We propose that this action of MPG is due to its 
strong thiol reductant properties, holding PKC $\varepsilon$ in a reduced state and thereby preventing its activation.

DMF blocks cardioprotection by ischemic preconditioning and diazoxide. The implication of the previous mitoK $\mathrm{K}_{\mathrm{ATP}}$ experiments is that intercepting the $\mathrm{HO}^{\circ}$ dependent reactions will block all modes of ROS-dependent cardioprotection. To examine this question, we perfused the heart with $1 \% \mathrm{DMF}$, the $\mathrm{HO} 0^{\circ}$-scavenger, following the protocols described in Fig. 2.3. As demonstrated in Fig. 2.10, DMF inhibited the improvement in post-ischemic functional recovery (Fig. 2.10A) and blocked the infarct-size reduction (Fig. 2.10B) normally observed with ischemic preconditioning (IPC) and diazoxide (Dzx). DMF alone had no effect on infarct size in these conditions (Fig. 2.10B). Again, the effect of DMF on cardioprotective signaling implicates a downstream oxidation product of $\mathrm{HO}^{\circ}$ as the signaling ROS.

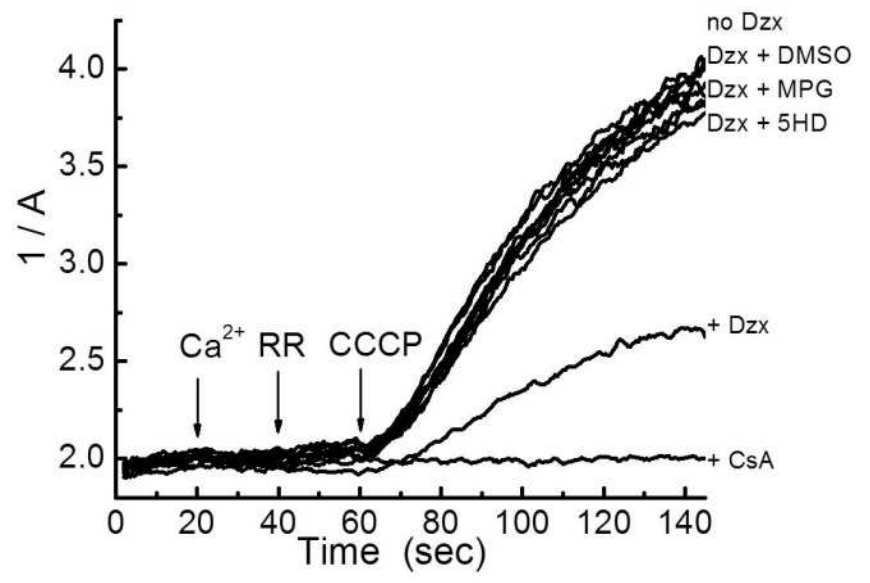

Fig. 2.11. Effects of free radical reactants on mito $_{\text {ATP-dependent MPT inhibition. Shown are light }}$ scattering traces $(1 / \mathrm{A})$ of rat liver mitochondria respiring on succinate in $\mathrm{K}^{+}$medium, and assayed as described in "Methods." Free calcium (100 $\mu \mathrm{M})$ ("Ca ${ }^{2+”), ~ r u t h e n i u m ~ r e d ~}(0.1 \mu \mathrm{M})$ ("RR"), and carbonyl cyanide $\mathrm{m}$-chlorophenylhydrazone $(0.25 \mu \mathrm{M})$

Endogenous ROS signaling and mitoK $K_{A T P}$-dependent MPT ("CCCP") were added sequentially at $20 \mathrm{sec}$ intervals, as shown. MPT inhibition by diazoxide (30 $\mu \mathrm{M})$ was blocked by 5 -HD $(300 \mu \mathrm{M})\left(\right.$ mitoK $_{\mathrm{ATP}}$ blocker), MPG (1 $\mathrm{mM})$ (thiolreductant), and DMSO $(1 \%, 14 \mathrm{M})\left(\mathrm{HO}^{\circ} / \mathrm{RO}^{\circ}\right.$ reactant). The data presented inhibition. MPT synchronization, are representative of three independent experiments with $p<0.05$. 
described in "Methods," was employed to determine the role of $\mathrm{HO}^{\circ}, \mathrm{RO}^{\circ}$ and/or $\mathrm{ROO}^{\circ}$ in diazoxide induced MPT inhibition, which depends on ROS activation of a second mitochondrial PKCE, PKC\&2 (18). Results of a representative experiment are contained in Fig. 2.11. CsA completely blocked MPT activity, and diazoxide ("Dzx") inhibited MPT opening by about $60 \%$, which is the customary extent observed in these experiments (20). This inhibition was reversed by 5-HD ("5HD"). MPG, DMSO, and DMF (not shown) also reversed MPT inhibition by diazoxide. These results also imply that the signaling ROS that activates PKC\&2 must be a downstream oxidation product of $\mathrm{HO}^{\circ}$. 


\section{2-E: Discussion}

The major findings of the present study are 1) that the signaling reactive oxygen species (ROS) responsible for activating mitochondrial PKCEs is a downstream oxidation product of hydroxyl radical, not superoxide or $\mathrm{H}_{2} \mathrm{O}_{2}$, and 2) that the main action of submillimolar MPG on mitochondria and perfused heart is inactivation of PKC $\varepsilon$ by thiol reduction rather than ROS scavenging.

MitoK $_{\text {ATP }}$ governs the ROS signaling that is essential for cardioprotection (40). MitoK $\mathrm{K}_{\mathrm{ATP}}$ opening has been found to increase levels of ROS in perfused heart (113), cardiomyocytes (109) and in the mitochondrial matrix (2). The ionophore valinomycin, at a concentration yielding the same $\mathrm{K}^{+}$flux as diazoxide, caused the same ROS increase as diazoxide, so mitoK $\mathrm{ATP}_{\mathrm{TP}}$-dependent increase in ROS is due specifically to $\mathrm{K}^{+}$influx into the matrix (2). The primary mitochondrial

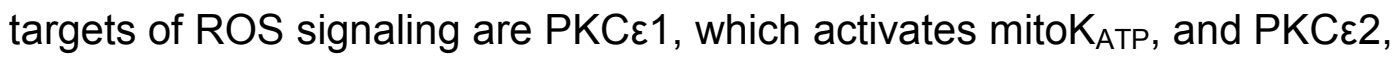
which inhibits MPT (reviewed in (45)). Mitochondrial PKCE1 and mitoK $\mathrm{ATP}_{\text {CO- }}$ purify and co-reconstitute to form a functioning signaling module in proteoliposomes (71). Thus, mitoK $\mathrm{ATP}_{\mathrm{i}}$ in either mitochondria or proteoliposomes can be opened by all PKCE activators, including phorbol esters, the specific peptide activator, $\Psi_{\varepsilon R A C K}$, and a variety of thiol oxidizing agents.

Our objective is to identify the mitoK $\mathrm{KTT}_{\mathrm{AT}}$-dependent endogenous ROS that activates PKC\&1 to leave mitoK $\mathrm{ATP}_{\mathrm{P}}$ in a phosphorylated open state. Superoxide is the first ROS that increases due to increased $\mathrm{K}^{+}$uptake into the matrix (see Fig. 2.1) and has been proposed to be the ROS signal (119). Xanthine/xanthine oxidase $(\mathrm{X} / \mathrm{XO})$ induced mitoK $\mathrm{K}_{\mathrm{ATP}}$ opening in isolated mitochondria; however, 
superoxide rapidly dismutates to $\mathrm{H}_{2} \mathrm{O}_{2}$ in aqueous solution, and Costa et al. (20) showed that $\mathrm{X} / \mathrm{XO}$ did not open mitoK $\mathrm{K}_{\mathrm{ATP}}$ in the presence of catalase. Therefore, the observed effect of $\mathrm{X} / \mathrm{XO}$ was mediated by $\mathrm{H}_{2} \mathrm{O}_{2}$, and superoxide can be excluded as a PKCE activator or mitoK $\mathrm{ATP}_{\mathrm{ATP}}$ opener. Queliconi, et al. (119) repeated the $\mathrm{X} / \mathrm{XO}$ experiment and obtained the same results; however they failed to control for $\mathrm{H}_{2} \mathrm{O}_{2}$ production and erroneously concluded that superoxide opens mitoK $\mathrm{ATT}_{\text {TP. }} \mathrm{PKC} \varepsilon$ is activated by nitric oxide (NO) (7) and $\mathrm{H}_{2} \mathrm{O}_{2}(82)$, which oxidize the thiols in the zinc finger of PKCE, leading to its activation. Consistent with this biochemistry, $\mathrm{H}_{2} \mathrm{O}_{2}$ and $\mathrm{NO}$ cause mitoK ATP opening $(20,71)$ that is blocked by the PKC $\varepsilon$ peptide inhibitor $\varepsilon \bigvee_{1-2}$. The absence of an effect of $\mathrm{H}_{2} \mathrm{O}_{2}$ and $\mathrm{NO}$ in the presence of $\varepsilon \bigvee_{1-2}$ demonstrates that these agents have no direct effect on mitoK $_{\text {AtP. }}$ Again, Quelconi, et al. (119) repeated these experiments and obtained the same result; however they failed to control for PKCE involvement and erroneously concluded that $\mathrm{H}_{2} \mathrm{O}_{2}$ and $\mathrm{NO}$ open mitoK

Liu, et al. (91) concluded that hydroxyl radical $\left(\mathrm{HO}^{\circ}\right)$ is responsible for ROS signaling in the heart, but this conclusion was based on experiments showing that MPG blocks ROS signaling and the assumption that this block was due to $\mathrm{HO}^{\circ}$ scavenging. This assumption may be incorrect. The data In Fig. 2.8 show that MPG blocks $\mathrm{H}_{2} \mathrm{O}_{2}$-induced mitoK $\mathrm{K}_{\text {ATP }}$ opening. $\mathrm{HO}^{*}$ is not involved in this process, and MPG has little or no $\mathrm{H}_{2} \mathrm{O}_{2}$ scavenging ability $(10,91)$. Moreover, eliminating $\mathrm{HO}^{\circ}$ requires very high concentrations of scavenger due to the transitory nature of $\mathrm{HO}^{\circ}$, and it is unlikely that sub-millimolar concentrations of MPG would be effective. Thus, most scavengers "will never be present at levels 
remotely approaching those of endogenous molecules that react at diffusioncontrolled rates with $\mathrm{HO}$ "” (63). Therefore, we infer that MPG prevents signaling, not by scavenging ROS, but rather by virtue of its powerful thiol reduction properties, keeping PKC $\varepsilon$ in a reduced, inactive state. Conclusions drawn from experiments in which MPG blocks ROS signaling, including those of Liu, et al. (91), should be re-evaluated in view of this new finding related to the mechanism of action of MPG.

To investigate the location giving rise to the endogenous ROS signal, we employed the preincubation protocol described in "Methods" and "Results". This protocol leads to feedback activation of mitoK $\mathrm{ATP}_{\mathrm{TP}}$, which depends on endogenous ROS signaling to PKCE1 (20). The results presented in Figs. 2.4 through 2.7 provide important clues to the identity of the ROS messenger. They are best understood in the context of Figs. 2.1 and 2.2. The antioxidants deferoxamine and Trolox each prevented mitoK $\mathrm{ATP}_{\mathrm{A}}$ opening. Deferoxamine is an iron chelator, and Trolox, a water soluble derivative of vitamin E, is a peroxyl radical scavenger and lipid peroxidation chain-breaking antioxidant. These data indicate that the redox-sensitive opening of mitoK $\mathrm{K}_{\mathrm{ATP}}$ is dependent on both iron and peroxyl radicals and are consistent with transition metal-catalyzed $\mathrm{ROO}{ }^{\circ}$ - dependent signaling. These steps occur downstream of $\mathrm{HO}^{\circ}$ production and strongly suggest that $\mathrm{HO}^{\circ}$ is the precursor of the signaling molecule. This is supported by the finding that inclusion of DMSO or DMF, which react with $\mathrm{HO}^{\circ}, \mathrm{RO}^{\circ}$, and/or $\mathrm{ROO}$ (5), in the preincubation prevented mitoK $\mathrm{ATP}_{\mathrm{AT}}$ opening. 
Many workers have proposed that $\underline{\mathrm{H}}_{2} \underline{\mathrm{O}}_{2}$ is the messenger of ROSdependent mitoK $\mathrm{K}_{\text {ATP }}$ opening $(2,145) . \mathrm{H}_{2} \mathrm{O}_{2}$ would appear to be a good candidate, not least because it is effective in activating mitochondrial PKC $\varepsilon$ (17, $20,71,154)$ and in protecting ischemic heart $(150,153)$ and hypoxic cardiomyocytes(31). However, a major finding of the present study is that $\mathrm{H}_{2} \mathrm{O}_{2}$ can be excluded as the signaling ROS in mitochondria and that the signaling ROS originates downstream of hydroxyl radical. The evidence is 1) the ROS signal arises downstream from $\mathrm{HO}^{\circ}$ radical, whereas $\mathrm{H}_{2} \mathrm{O}_{2}$ is produced upstream of $\mathrm{HO}^{\circ}$ radical (Fig. 2.1); 2) catalase, the $\mathrm{H}_{2} \mathrm{O}_{2}$ scavenger, had no effect on the formation of the phosphorylation-dependent open state, whereas DMF and DMSO, which have neither $\mathrm{H}_{2} \mathrm{O}_{2}$ scavenging nor thiolreductant properties, do block PKCE-dependent mitoK ATP $_{\text {TP }}$ activation. Circumstantial evidence also argues against $\mathrm{H}_{2} \mathrm{O}_{2}$ as the signaling ROS: $\mathrm{H}_{2} \mathrm{O}_{2}$ is produced in the matrix, whereas PKCE1 faces the intermembrane space. The matrix of heart mitochondria contains glutathione, thioredoxin reductase and catalase (121), which may prevent sufficient $\mathrm{H}_{2} \mathrm{O}_{2}$ from reaching its target (132).

The conclusion that the ROS message is formed downstream from $\mathrm{HO}^{*}$ is further supported by the effects of DMSO and DMF on endogenous ROSdependent MPT inhibition (Fig. 2.10). Finally, the finding that DMF prevents cardioprotection by both ischemic preconditioning (IPC) and diazoxide (Fig. 2.11) supports extension of the conclusion to the ex vivo and in vivo heart. That $\mathrm{HO}^{\circ}$ is necessary for cardioprotective signaling should not be taken to mean that $\mathrm{HO}^{\circ}$ 
itself is the signaling ROS. $\mathrm{HO}^{\circ}$ is too reactive for signaling, with a half-life that is diffusion-limited at about one nanosecond (63). $\mathrm{HO}^{\circ}$ will react with its nearest neighbors almost immediately after it is formed, allowing for the formation of downstream oxidation products that are most likely responsible for signaling.

Finally, the finding that BEL interrupted the ROS signal (Fig. 2.7) implicates hydroperoxy fatty acids $(\mathrm{FAOOH})$ as the ROS signal. A full investigation of the effects of fatty acids and hydroperoxy fatty acids on mitoK $\mathrm{K}_{\text {ATP }}$ and on ischemia-reperfusion injury will be presented in a subsequent communication.

Limitations. 1) The conclusions are based largely on in vitro experiments carried out on isolated mitochondria. The rationale for this approach is that the signaling ROS not only originates in mitochondria but also acts on mitochondria. We have no reason to believe that the ROS transformation reaction sequence is any different in vivo. 2) We stress that the conclusions relate specifically to ROS signaling secondary to mitoK $\mathrm{K}_{\mathrm{ATP}}$ opening. The reverse sequence - mitoK $\mathrm{K}_{\mathrm{ATP}}$ opening caused by ROS - may also be physiologically important. For example, it is possible that the increased ROS that occurs during ischemia opens mitoK $\mathrm{ATP}_{\text {, }}$ and this ROS may arise from a different location, such as Complex III. The identity of the ROS signal in this setting has not yet been addressed. 3) BEL has been shown to inhibit activation of store-activated $\mathrm{Ca}^{2+}$ channels; however this is mediated by inhibition of iPLA2 and is therefore a pharmacological effect (130). It has recently been shown that $\mathrm{BEL}$ inhibits voltage gated $\mathrm{Ca}^{2+}$ and transient receptor potential canonical channels independently of iPLA2 (14). We consider 
it unlikely that these effects play a role in the effect of BEL on isolated mitochondria.

\section{Conclusions and potential conceptual/pragmatic values of these}

findings. We conclude that the signaling ROS responsible for activating mitochondrial PKCEs is a downstream oxidation product of hydroxyl radical and that superoxide and $\mathrm{H}_{2} \mathrm{O}_{2}$ are not the signaling ROS. We further conclude that the main action of submillimolar MPG on mitochondria and perfused heart is not ROS scavenging, but rather thiol reduction, causing inactivation of PKC $\varepsilon$ and other ROS-dependent kinases. MitoK $\mathrm{ATP}_{\mathrm{A}}$-dependent ROS signaling is a central process in cardioprotection and cellular signaling generally. Determining the identity of the mitochondrial signaling ROS is important for future research in these areas. 


\section{ACKNOWLEDGMENTS}

The authors express gratitude for the excellent technical and administrative assistance of Craig Semrad, Caitlin E. Pesout and Beau Sober.

\section{GRANTS}

This work was supported by grants HL35673 and HL067842 from the National Heart, Lung and Blood Institute to K.D. Garlid, and by grant ME09018 to M. Jaburek.

\section{DISCLOSURES}

No conflicts of interest, financial or otherwise, are declared by the author(s).

\section{AUTHOR CONTRIBUTIONS}

AOG, MJ and KDG conceived and designed experiments and wrote the paper. AOG, MJ and JPJ performed the experiments and analyzed the results. 
Chapter 3: The role of fatty acids and phospholipase iPLA2 in mitochondrial ATP-sensitive $\mathrm{K}^{+}$channel activity and cardioprotection

\author{
Martin Jaburek ${ }^{1}$, Anders O. Garlid ${ }^{2}$, Petr Jezek ${ }^{1}$ and Keith D. Garlid ${ }^{2 *}$ \\ ${ }^{2}$ Department of Biology, Portland State University, Portland, OR, 97201-0751, \\ 1Department of Membrane Transport Biophysics, Institute of Physiology, v.v.i., \\ Prague, Czech Republic, 14220 \\ Running Head: Hydroperoxy fatty acids are Second Messengers of \\ Cardioprotection
}

Keywords: mitochondria; cardioprotection; hydroperoxy fatty acids; K-ATP channels; cardiac ischemia; ROS signaling 


\section{3-A: Abstract}

During ischemia, catabolism of mitochondrial phospholipids is accelerated, causing accumulation of plasmalogens and free fatty acids (FA) in the heart. Calcium-independent phospholipases A2 (iPLA2) are the major phospholipases in myocardium and the role of iPLA2 as well as the effects of various fatty acids and fatty acid analogues during ischemia are not completely understood. Because mitochondrial ATP-sensitive $\mathrm{K}^{+}$channel (mitoK $\mathrm{ATP}_{\mathrm{A}}$ ) is known to be involved in cardioprotection, here we tested whether FAs affect mitoK $\mathrm{ATP}_{\mathrm{P}}$ opening and cardioprotection. We also studied whether there is a protective role of iPLA2mediated FA release and tested our hypothesis that hydroperoxy fatty acids $(\mathrm{FAOOH})$ are the second messengers of cardioprotective reactive oxygen species (ROS)-mediated signaling. Following the changes in mitoK $\mathrm{ATP}_{\text {-dependent }}$ changes in mitochondrial matrix volume, our results indicate that natural fatty acids and their hydroxy derivatives inhibit the pharmacologically open state of mitoK $_{\text {ATP. }}$ Our results also show that fatty acids inhibit the phosphorylationdependent open state of mitoK $\mathrm{K}_{\mathrm{ATP}}$ with lower affinity than the pharmacologically open state. We found that hydroperoxylinoleic acid opens the ATP-inhibited mitoK $K_{\text {ATP }}$ and that its effect is blocked by the specific PKC $\varepsilon$ inhibitor peptide $\varepsilon \bigvee_{1-2}$. These data support our hypothesis of direct activation of PKC $\varepsilon$ by FAOOH. Using Langendorff perfused hearts, our studies indicate that iPLA2 is essential for most modes of cardioprotection. We show that a selective inhibitor of $\mathrm{PPLA} 2$, racemic bromoenol lactone (BEL), is cardioprotective when added before the ischemia and reperfusion. These results are consistent with BEL protecting against 
ischemic reperfusion injury by preventing ischemic release of free fatty acids. However, our result also show that BEL blocks protection by preconditioning and postconditioning when added with the treatment. We interpret these results as the ability of BEL to prevent the iPLA2-dependent release of $F A O O H$, which are necessary for protection.

Keywords: mitochondria; cardioprotection; hydroperoxy fatty acids; K-ATP channels; cardiac ischemia; ROS signaling 


\section{3-B: Introduction}

Mitochondria are effectors of both ischemia-reperfusion injury (IRI) and cardioprotection. Ischemia causes profound and immediate mitochondrial derangements, including cessation of ATP synthesis, inhibition of respiration, and a drop in membrane potential $(\Delta \Psi)$. This is accompanied during ischemia by increases in $\mathrm{Ca}^{2+}$ and phosphate, and during reperfusion, by large increases in reactive oxygen species (ROS) $(30,141)$. Together, these factors promote opening of the mitochondrial permeability transition (MPT), a high-conductance pore in the inner mitochondrial membrane, which is the main cause of necrotic cell death in IRI $(22,24,25,65,148)$.

In addition to their role as mediators of cell death in IRI, mitochondria are major effectors of self-defense mechanisms, including ischemic pre- and postconditioning $(3,4,53,65)$. These and other conditioning protocols have been shown to require opening of the mitochondrial ATP-sensitive $\mathrm{K}^{+}$channel (mitoK $\mathrm{ATP}_{\text {). }}$. Considerable progress has been made in understanding the mechanisms by which mitoK $\mathrm{K}_{\mathrm{ATP}}$ opening increases ROS production (2) and the

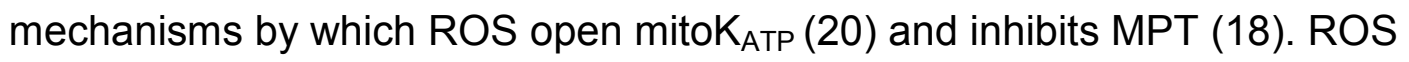
activate key protein kinases (19), making them the second messengers of preconditioning (23). We described data in myocytes supporting the hypothesis that mitoK $\mathrm{K}_{\mathrm{ATP}}$ opening was responsible for the increased ROS of cardioprotection (40), and this was confirmed by several laboratories $(32,40,84,108,110,111$, 138). 
During ischemia, catabolism of mitochondrial phospholipids is accelerated, causing accumulation of plasmalogens and fatty acids $(F A)$ in the heart $(89,142$, 143). Mitochondrial cardiolipin suffers the most profound change, its level decreasing by $30-50 \%$ (112). Several reports have highlighted the negative role that fatty acids may play in cardioprotection. For example, Ford, et al. (33) found that cromakalim did not protect working rat hearts that were perfused with fatty acids. Ischemia itself causes fatty acid accumulation in the heart, caused by net degradation of membrane phospholipids by phospholipases $(142,143)$. Prasad, et al. (118) and Harris, et al. (64) found that inhibition of phospholipase was cardioprotective. In important work, Sargent, et al (124) and Williams and Gottlieb (149) found that perfusion of rabbit hearts with bromoenol lactone (BEL) prior to ischemia reduced the infarct size almost to that of controls not subjected to ischemia. Protection by BEL was reversed by the simultaneous perfusion of 5hydroxydecanoic acid (5-HD), implicating participation of mitoK $\mathrm{ATP}_{\mathrm{TP}}(125)$. Importantly, BEL is a specific inhibitor of calcium-independent phospholipase A2 (iPLA2), and iPLA2 $y$ is the major phospholipase A2 in myocardium $(13,73)$.

Almost all of the attention on iPLA2 has focused on its deleterious effects (149), due to its releasing free fatty acids into the heart during ischemia. Thus, the iPLA2 inhibitor bromoenol lactone (BEL), administered before global ischemia, is cardioprotective in rat (124) and rabbit (149), causing marked reduction of infarct size. Protection by BEL was reversed by the simultaneous perfusion of 5-HD, implicating participation of mitoK $\mathrm{K}_{\mathrm{ATP}}$. FA have long been known to promote MPT opening $(129,133)$, and BEL was also found to inhibit 
$\mathrm{Ca}^{2+}$-dependent MPT opening, due to inhibition of IPLA2-mediated arachidonic acid release (80).

There are several studies, however, that point to the protective effect of fatty acids, namely the protective effects of the products of lipoxygenase metabolism. Lipoxygenase inhibitors were shown to block the protective effect of preconditioning and elevated levels of 12-hydroxyeicosatetraenoic acid, a stable product of the lipoxygenase pathway of arachidonic acid metabolism, were detected following the preconditioning protocol (100). There is a correlation between functional recovery after ischemia and stimulation of the lipoxygenase pathway before the sustained period of ischemia (16) and experiments using leukocyte-type 12lipoxygenase (12-LOX) deficient mice demonstrated the importance of $12-$ LOX in preconditioning (35).

Given the complex effects of various fatty acids during ischemia, we investigated the interactions of selected fatty acids with mitoK $\mathrm{K}_{\text {ATP. }}$ We tested whether fatty acids other than

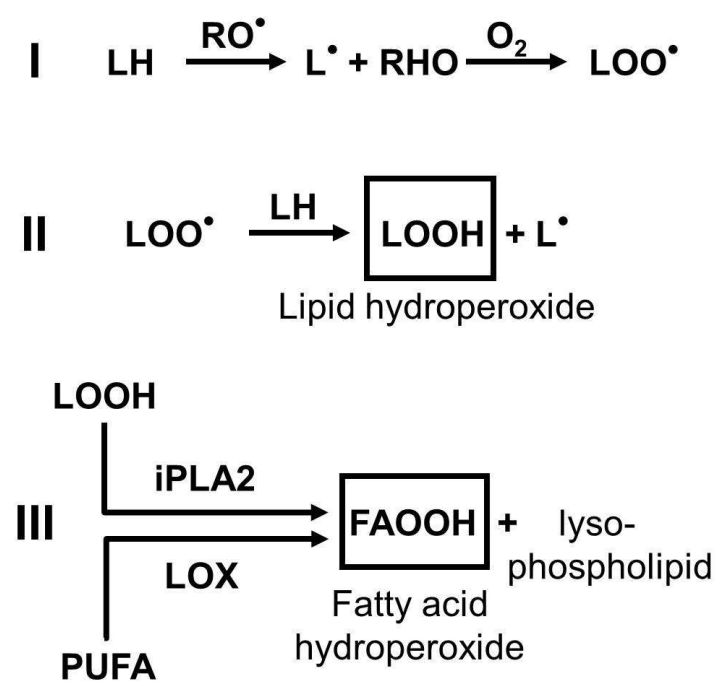

Fig 3.1. Lipid peroxidation and release of hydroperoxy fatty acids. (I and II) alkoxyl $\left(\mathrm{RO}^{\circ}\right)$ and alkyl peroxyl radicals (LOO') and alkyl hydroxyl molecules (RHO) can initiate the non-enzymatic chain reaction with fatty acid side chains (LH), leading to lipid hydroperoxides (LOOH). (III) $\mathrm{LOOH}$ can be released by the action of mitochondrial phospholipases, such as $\mathrm{Ca}^{2+}$-independent phospholipases PLA2 (iPLA2), which have been found to modulate the function of mitochondria (see Cedars et al (13) for a review). The action on LOOH by iPLA2 results in a hydroperoxy fatty acid (FAOOH). Alternatively, FAOOH are formed from polyunsaturated fatty acids (PUFA) by the action of lipoxygenases (LOX). 
5-HD affect mitoK $\mathrm{K}_{\text {ATP }}$ opening and cardioprotection. We have further studied the possible protective role of iPLA2-mediated FA release and tested our hypothesis that hydroperoxy fatty acids $(\mathrm{FAOOH})$ are the second messenger of ROS signaling. Using the light scattering technique, our results indicate that natural fatty acids and their hydroxy derivatives behave similarly to 5-HD and inhibit the pharmacologically open state of mitoK $\mathrm{ATP}_{\text {. }}$ Our results also show that fatty acids inhibit the phosphorylated open state of mitoK $\mathrm{K}_{\text {ATP }}$ with lower affinity than the pharmacologically open state. We found that hydroperoxylinoleic acid, LAOOH, opens the ATP-inhibited mitoK $\mathrm{ATP}_{\text {at }}$ and that its effect is blocked by the specific PKC $\varepsilon$ inhibitor peptide $\varepsilon \bigvee_{1-2}$. These data support our hypothesis of direct activation of PKCE by FAOOH. Our studies using Langendorff perfused heart indicate that iPLA2 is essential for most modes of cardioprotection. We show that BEL inhibits both ischemic preconditioning (IPC) and postconditioning (PC) when added with the treatment. These results are consistent with BEL protecting against ischemic reperfusion injury by preventing ischemic release of free fatty acids. However, our results also show that BEL blocks protection by IPC and postconditioning. We interpret these results as the ability of BEL to prevent the iPLA2-dependent release of $\mathrm{FAOOH}$, which are necessary for protection. 


\section{3-C: Research Design and Methods}

\section{Langendorff perfused hearts}

Hearts from male Sprague-Dawley rats (200-240g) were perfused for 50 55 min as previously described $(51,115,120)$ with Krebs Henseleit buffer containing (in mM) $118 \mathrm{NaCl}, 5.9 \mathrm{KCl}, 1.75 \mathrm{CaCl}_{2}, 1.2 \mathrm{mM} \mathrm{MgSO}_{4}, 0.5$ EDTA, 25 $\mathrm{NaHCO}_{3}, 16.7$ glucose at $\mathrm{pH} 7.4$ and gassed with $95 \% \mathrm{O}_{2}-5 \% \mathrm{CO}_{2}$. Hearts were stabilized for 25 min with Krebs Henseleit buffer, then with buffer containing drugs or agents, and then subjected to 25 minutes global ischemia followed by 2 hours of reperfusion and measurement of infarct size. Ischemic preconditioning (IPC) was established by two cycles of $5 \mathrm{~min}$ global ischemia followed by $5 \mathrm{~min}$ reperfusion prior to the index ischemia. Ischemic postconditioning was performed with 6 cycles of $10 \mathrm{~s}$ ischemia plus $10 \mathrm{~s}$ reperfusion (140). Cross-sectional slices were stained with TTC after each treatment, scanned, and analyzed to report infarct size as the percentage of damaged tissue relative to the area at risk (IS \% Area at Risk). Treatments deemed protective were found to exhibit a reduced infarct size to an extent similar to that reported elsewhere $(85,114,140)$. Experimental protocols were treated in accordance with the Guiding Principles in the Use and Care of Animals published by the National Institutes of Health.

\section{Mitochondrial isolation}

Male Sprague-Dawley rats (200 - $240 \mathrm{~g}$ ) were anesthetized with $\mathrm{CO}_{2}$ and immediately decapitated. The hearts were removed and washed in ice-cold Buffer A (250 mM sucrose, $10 \mathrm{mM}$ HEPES pH 7.2, and 2 mM K-EGTA). The 
tissue was finely minced in the presence of $1 \mathrm{mg} / \mathrm{ml}$ protease (type XXIV Sigma), and the suspension was diluted 3-fold with Buffer A supplemented with $1 \mathrm{mg} / \mathrm{ml}$ fatty acid-free BSA. We observed that mitoK $\mathrm{ATP}_{\mathrm{T}}$ activity depends critically on the time between sacrifice and completion of homogenization. This period was kept as brief as possible and was completed within $2 \mathrm{~min}$. The suspension was homogenized with a motorized Teflon pestle and centrifuged for 3 min at $1500 \mathrm{~g}$. The supernatant was centrifuged for $5 \mathrm{~min}$ at $9000 \mathrm{~g}$, and the resulting pellets were resuspended in Buffer A lacking BSA and centrifuged for 3 min at $2300 \mathrm{~g}$. This supernatant was centrifuged for $5 \mathrm{~min}$ at $9000 \mathrm{~g}$. The final mitochondrial pellet was resuspended at $35-40 \mathrm{mg}$ protein/ $\mathrm{ml}$ and kept on ice. Mitochondrial protein concentration was estimated using the Biuret reaction (54). This procedure is in accordance with the Guiding Principles in the Care and Use of Animals and was approved by IACUC at Portland State University.

\section{Bromoenol lactone treatment of mitochondria}

Where indicated, the mitochondrial pellet was treated with racemic bromoenol lactone (BEL) to inhibit mitochondrial phospholipase iPLA2 and avoid unappreciated accumulation of free fatty acids during the course of a given experiment. The pellet was resuspended in $1 \mathrm{ml}$ of Buffer A lacking BSA in the presence of $5 \mu \mathrm{M}$ BEL. The suspension was diluted to $30 \mathrm{ml}$ with Buffer $\mathrm{A}$ supplemented with $0.5 \%$ fatty-acid free BSA to remove residual fatty acids and centrifuged $9000 \mathrm{~g}$ for 5 minutes. The resulting pellet was resuspended and the protein concentration was determined as stated above. 


\section{Preincubation protocol of isolated mitochondria}

Where indicated, isolated mitochondria were treated by an additional preincubation procedure aimed to investigate mitochondria under the conditions of endogenous signaling ROS (20). The mitochondria were incubated in assay medium for 3 minutes at $30^{\circ} \mathrm{C}$ with $200 \mu \mathrm{M}$ ATP and $30 \mu \mathrm{M}$ diazoxide (Dzx). The mitochondria were then isolated from the incubation medium and resuspended in fresh assay medium. MitoK $\mathrm{K}_{\mathrm{ATP}}$ activity was observed using the light scattering assay, as described below, with ATP in the presence or absence of diazoxide.

Measurements of mitochondrial ATP-sensitive $K^{+}$channel

Changes in mitochondrial matrix volume, which accompany net salt transport into mitochondria, were followed using a quantitative light scattering technique $(9,43)$. Mitochondria were suspended in a buffered salt assay medium containing $\mathrm{K}^{+}$salts of $\mathrm{Cl}^{-}(120 \mathrm{mM})$, HEPES (10 mM), EGTA (0.1 mM), succinate $(10 \mathrm{mM})$ and phosphate $\left(\mathrm{P}_{\mathrm{i}}\right)(5 \mathrm{mM}), \mathrm{pH}$ 7.2. The osmolality of these media ranged between 275 to $280 \mathrm{mOsm}$. All media also contained $0.5 \mathrm{mM} \mathrm{MgCl}_{2}, 2.5$ $\mu \mathrm{M}$ rotenone and $1 \mu \mathrm{g} / \mathrm{ml}$ oligomycin.

Light scattering changes of $0.1 \mathrm{mg} / \mathrm{ml}$ mitochondrial suspensions were followed at $520 \mathrm{~nm}$ and $30^{\circ} \mathrm{C}$. The data collection was initiated by addition of the mitochondrial suspension. Data summarized in bar graphs as "MitoK $\mathrm{ATP}_{\text {activity }}$ (\%) @ $\mathrm{t}=0 \mathrm{~s}$ " are given by

$$
100 \times \frac{[V(x)-V(A T P)]}{[V(0)-V(A T P)]}
$$


where $V(x)$ is the observed volume at $120 \mathrm{~s}$ under the given experimental condition and $V(A T P)$ and $V(0)$ are observed values in the presence and absence of ATP, respectively.

Statistical analysis

Data are presented as mean \pm standard deviation (SD) of the mean. Where appropriate, one-way ANOVA or repeated measures $t$-test were used to analyze the data using Microcal Origin software (Northampton, MA, USA). A value of $p<0.05$ was considered statistically significant.

\section{Chemicals}

All fatty acids, fatty acid derived compounds and bromoenol lactone were from Cayman Chemical. All other chemicals used in the study were from Sigma Chemical Co. (St. Louis, MO, USA). 


\section{3-D: Results}

5-hydroxydecanoic acid (5-HD) is a relatively simple fatty acid which is considered a specific inhibitor of the mitochondrial ATP-sensitive potassium channels (mitoK $\mathrm{ATP}_{\mathrm{A}}$ (125). However, its chemical structure suggests that its action should not differ greatly from that of other fatty acids. We undertook to investigate this, because the actions of fatty acids and their hydroxy and hydroperoxy derivatives on mitoK $\mathrm{K}_{\mathrm{ATP}}$ have not been previously investigated. Fig.

3.2 shows light scattering traces from heart mitochondria respiring in $\mathrm{K}^{+}$medium. The control traces in the absence of ATP and in the presence of ATP indicate the

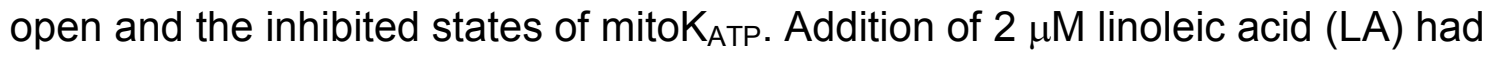

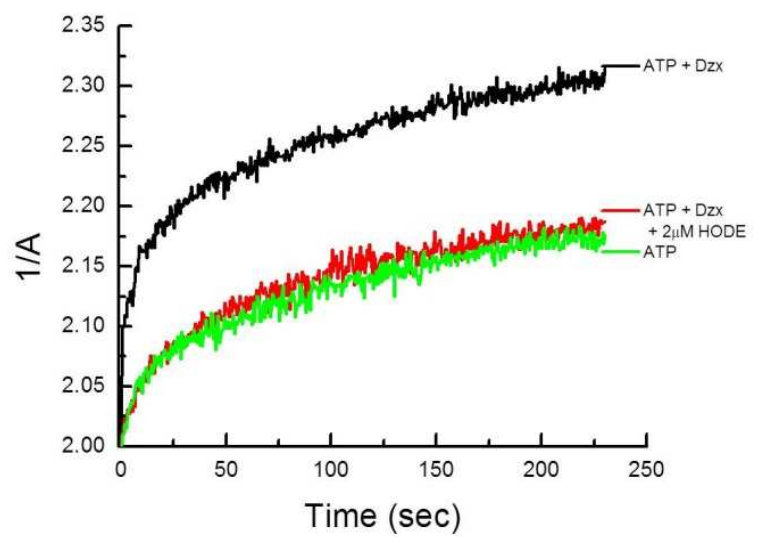

Fig. 3.2. 13-(S)-HODE inhibits mitoK $K_{A T P .}$ Light scattering traces from heart mitochondria respiring in $\mathrm{K}^{+}$medium. The control trace in the in the presence of 0.2 mM ATP indicates the inhibited (closed) state of mitoK $\mathrm{KTP}_{\mathrm{P}}$ and the addition of $30 \mu \mathrm{M}$ diazoxide (ATP + Dzx) establishes the open state. Addition of $1 \mu \mathrm{M}$ 13-(S)-HODE had no effect on the control traces in the absence or presence of ATP respectively (not shown), but blocked the diazoxide-induced open state in the presence of ATP (ATP + Dzx + HODE). These are representative traces of at least 3 independent experiments. no effect on the control traces in the absence or presence of ATP respectively (data not shown), but had an effect on the open trace in the presence of ATP and the Pharmacological opener diazoxide, similar to the known properties of 5HD. Because 5-HD, the known inhibitor of mitoK $\mathrm{K}_{\mathrm{ATP}}$, is a fatty acid containing a hydroxy group, we tested whether the hydroxy derivative of linoleic acid exhibits similar effects. 
The data in Fig. 3.2 show that the hydroxy derivative of linoleic acid, 13hydroxyoctadecanoic acid (13-(S)-HODE, $0.5 \mu \mathrm{M})$, inhibits mitoK $\mathrm{ATP}_{\text {in }}$ in the presence of ATP and diazoxide. Similar sets of data were obtained with other fatty acids, including lauric acid, 12-hydroxylauric acid, and oleic acid (data not shown). The data indicate that natural fatty acids and their hydroxy derivatives behave similar to $5-\mathrm{HD}$ and inhibit the pharmacologically open state of mitoK $\mathrm{K}_{\mathrm{ATP}}$. Linoleic acid inhibited the pharmacologically-opened state of mitoK $\mathrm{K}_{\text {ATP }}$ in the presence of ATP and diazoxide with a half-maximal inhibition of $0.46+/-0.06$ $\mu \mathrm{M}(\mathrm{n}=3)$. To avoid unappreciated changes in the mitochondrial matrix volume due to adenine nucleotide translocase (ANT) - dependent, fatty acid-mediated decrease in membrane potential, similar sets of data were obtained in the presence of carboxyatractyloside (CAT), an inhibitor of the ANT. In the presence of CAT, the half-maximal inhibition was $0.6+/-0.05 \mu$ M linoleic acid $(n=3)$, indicating that the ANT-dependent processes did not significantly contribute to the changes in mitochondrial matrix volume under the given experimental conditions.

Hydroxy fatty acids are the detectable stable byproducts of fatty acid peroxidation that are formed by the enzymatic action of lipid glutathione peroxidases (68). Therefore, the stimulating effect of hydroperoxy fatty acids $(\mathrm{FAOOH})$ on mitoK $\mathrm{ATP}_{\mathrm{T}}$ would be hindered by the inhibitory effect of inherently formed hydroxy fatty acids, if the affinity of mitoK $\mathrm{ATP}_{\mathrm{P}}$ to inhibitory fatty acids remains unaltered. On that account we speculated that the phosphorylated open state of mitoK $\mathrm{K}_{\text {ATP }}$ must be less prone to the inhibition by fatty acids and hydroxy fatty acids. To explore this hypothesis, we tested the effect of 13-(S)-HODE on 


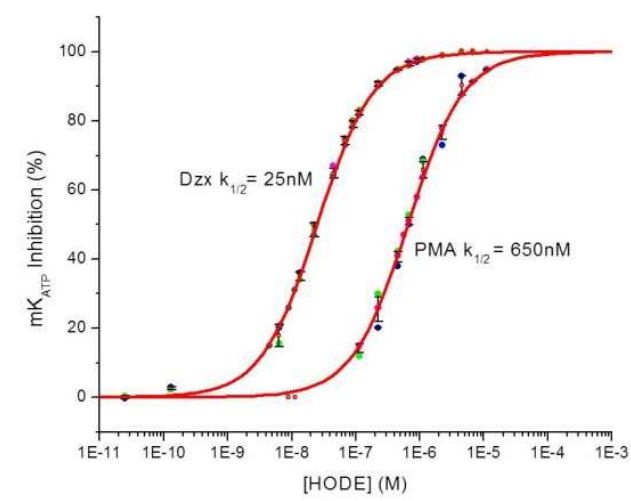

Fig. 3.3. Diazoxide exhibits a $\sim 25$ fold greater sensitivity to 13-(S)-HODE than $P M A$. The dose response curves demonstrate that pharmacological mitoK $\mathrm{K}_{\mathrm{ATP}}$ opening is much more sensitive to inhibition by fatty acids than physiological, $\mathrm{PKC} \varepsilon-$ dependent opening. HODE exhibited a $\mathrm{K}_{1 / 2}$ of against the general PKC activator phorbol 12-myristate-13-acetate (PMA) while it exhibited a $\mathrm{K}_{1 / 2}$ of with the $\mathrm{K}^{+}$-channel opener diazoxide. Data are representative of at least 5 independent experiments. the mitoK $\mathrm{K}_{\mathrm{ATP}}$ in the presence of the pharmacological opener diazoxide or in the presence of general PKC activator phorbol 12-myristate 13acetate (PMA). As seen in Fig. 3.3, 13-(S)-HODE inhibits the pharmacological open state of mitoK $_{\text {ATP }}$ induced by diazoxide and Fig. 3.3 demonstrates that it inhibits diazoxide with $\sim 25$-fold greater affinity than the PMA.

We have also preincubated the

mitochondria using our previously described preincubation protocol (20), except the pharmacological opener diazoxide was replaced by the general PKC activator phorbol myristate acetate (PMA). After preincubating mitochondria with PMA, 13-(S)-HODE inhibits mitoK $\mathrm{ATP}_{\mathrm{T}}$ with significantly lower affinity than observed for its inhibition of the pharmacologically open state. Similar data were obtained when using LA and arachidonic acid as the inhibitory FA (data not shown). These experiments indicate that fatty acids inhibit the phosphorylated open state of mitoK $\mathrm{ATP}_{\mathrm{T}}$ with significantly lower affinity than the pharmacologically opened state. 
Figs. 3.5A and 3.5B show the

effects of 13-(S)-HODE in

Langendorff-perfused hearts. Fig. 3.4

depicts the Langendorff perfusion

protocols used. Both ischemic

preconditioning (IPC) and diazoxide

$(50 \mu \mathrm{M})$, the pharmacological

mitoK $_{\text {ATP }}$ opener, significantly reduce

myocardial infarct sizes and improve

functional recovery compared with

untreated ischemic hearts. The

protective effects of both were

abolished by concurrent treatment
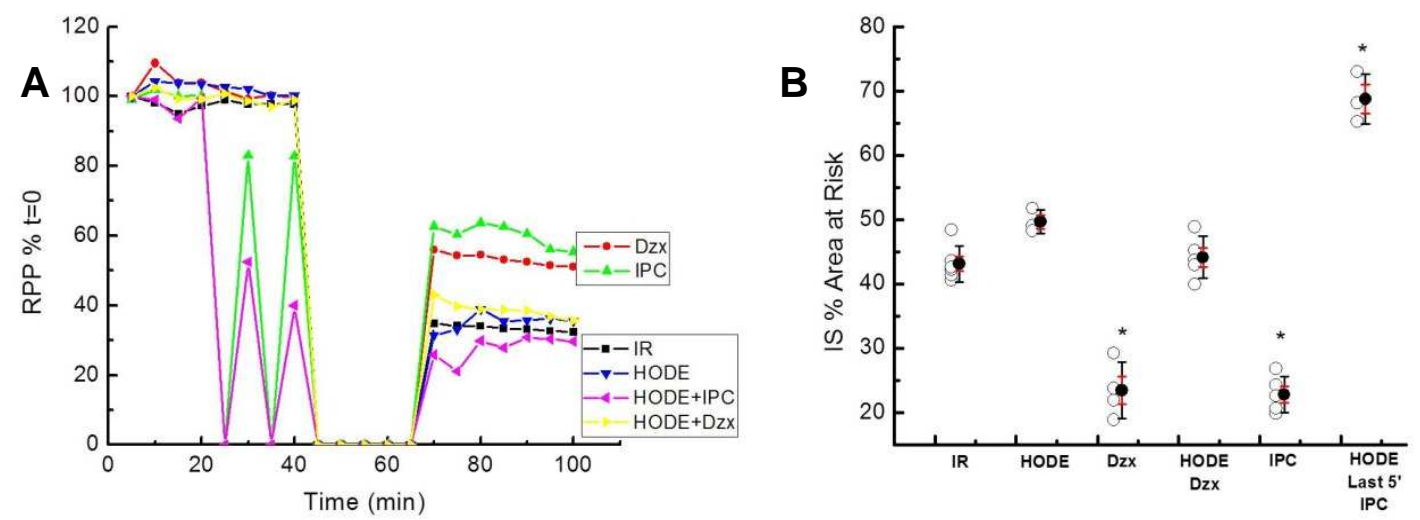

Fig. 3.5. 13-(S)-HODE blocks IPC and Dzx protection in isolated perfused rat heart. The graph in (A) shows that HODE blocks the improvement in functional recovery afforded by both diazoxide (Dzx) and IPC. These are representative traces of at least 3 independent experiments. The chart in (B) shows that the infarct size reduction from diazoxide and IPC is also blocked by HODE. This data contains results from at least 3 independent experiments and data are means $\pm S D ;{ }^{*} p<0.05$. 
with $2 \mu \mathrm{M}$ 13-(S)-HODE.

Our previous results have established $\mathrm{HO}^{\circ}$ as a key component of the

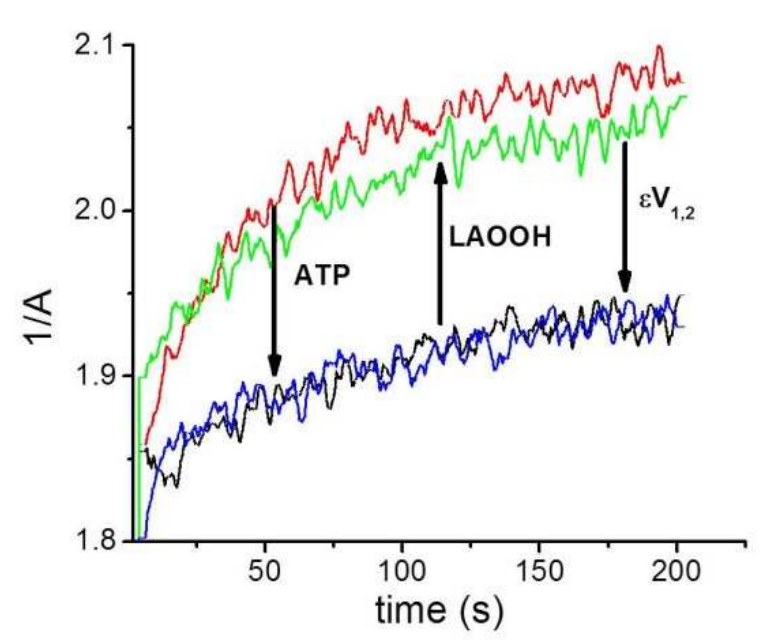

Fig. 3.6. Hydroperoxy fatty acid-dependent opening of mito $K_{A T P}$ is mediated via PKCE. Light scattering traces (1/A) of rat heart mitochondria respiring on succinate in $\mathrm{K}^{+}$ medium. With the exception of the trace Ano ATP@, ATP was present in all traces. $\mathrm{FAOOH}$ opens mitoK $\mathrm{KTT}_{\mathrm{T}}$ in a $\mathrm{PKC} \varepsilon-$ dependent manner, as demonstrated by its inhibition by $\varepsilon \mathrm{V}_{1-2}$. Mitochondria were suspended at $0.1 \mathrm{mg} / \mathrm{ml}$ and assayed as described in AMethods@. LAOOH $(1 \mu \mathrm{M})$ was added 2 seconds after the addition of mitochondria. ATP $(0.2 \mathrm{mM})$ and $\varepsilon \mathrm{V}_{1-2}(1 \mu \mathrm{M})$ were present in the assay medium prior to the addition of mitochondria. These results are representative of at least 3 independent experiments. cardioprotective signaling (36). $\mathrm{HO}^{\circ}$ is very unlikely to play a direct role in signaling, however, because its halflife is short and it reacts with everything it meets. Therefore, we have hypothesized that a downstream product that may arise from an increase in $\mathrm{HO}^{\circ}$ is responsible for the signaling (36). Hydroperoxy fatty acids $(\mathrm{FAOOH})$ are transient, non-radical and reactive species (27) that are known to activate protein kinases (134). They have the distinct advantage of being able to flip-flop across the inner membrane bilayer to

reach $\mathrm{PKC} \varepsilon$ on either side of the membrane (72). In the following sets of experiments, we aimed to determine whether $\mathrm{FAOOH}$ are competent ROS messengers.

Fig. 3.6 contains light scattering traces from heart mitochondria respiring in $\mathrm{K}^{+}$medium showing that $1 \mu \mathrm{M}$ hydroperoxy linoleic acid (13- 
hydroperoxyoctadecanoic acid, or $\mathrm{LAOOH}$ ), a product of cardiolipin peroxidation and cleavage, had no effect on mitoK $\mathrm{ATP}_{\mathrm{P}}$ under the conditions used to demonstrate that fatty acids inhibit the channel. That is, in the presence of ATP and the pharmacological opener diazoxide, $\mathrm{LAOOH}$ was ineffective. To the contrary, $\mathrm{LAOOH}$ was able to open the ATP-inhibited mitoK $\mathrm{ATP}_{\text {TP. The data also }}$ show that $\mathrm{LAOOH}$ acts via $\mathrm{PKC} \varepsilon$, because its effect was blocked by the specific inhibitor peptide $\varepsilon \bigvee_{1-2}$. The data support our hypothesis of direct activation of PKC $\varepsilon$ by hydroperoxy fatty acids in general.

That hydroperoxy fatty acids open mitoK $\mathrm{K}_{\mathrm{ATP}}$ suggests that they also protect the heart. Indeed, Fig. $\mathbf{3 . 7}$ demonstrates that the high infarct size observed with an ischemia-reperfusion (IR) is abolished by diazoxide (Dzx) and ischemic preconditioning (IPC) and that $\mathrm{LAOOH}$ causes a nearly identical reduction in infarct size; each of these treatments also showed significant improvement in the functional recovery of the hearts (data not shown).

We are not certain which of the many products of phospholipid oxidative modifications and breakdown are responsible for the signaling in vivo. For example, 4-hydroxynonenal

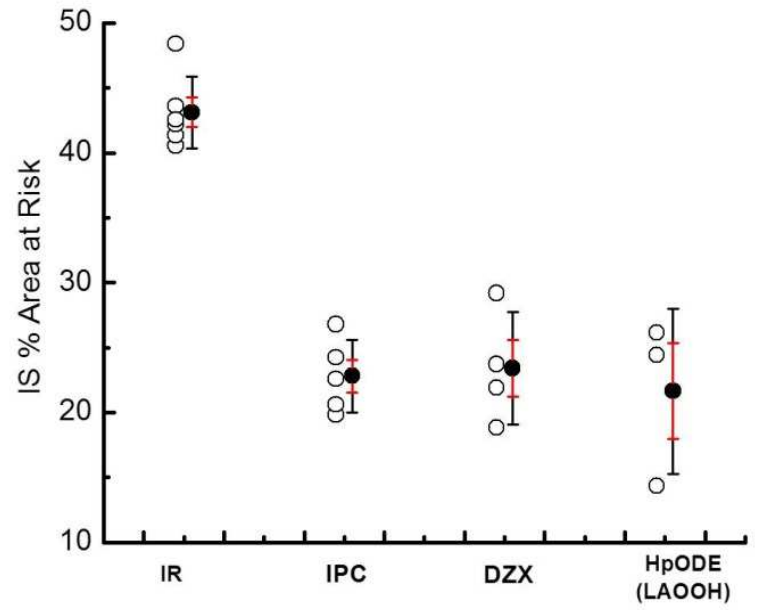

Fig. 3.7. $F A O O H$ confer protection to the same degree as IPC and diazoxide. $\mathrm{FAOOH}$ perfused at $2 \mu \mathrm{M}$ were cardioprotective in the isolated perfused rat heart, to the same degree as ischemic preconditioning (IPC) and diazoxide (Dzx). Each treatment resulted in an improvement in functional recovery (not shown) and a significant reduction in infarct size. Data are means $\pm S D$ of at least 3 independent experiments and significant with $p<0.05$. 
(HNE), a non-enzymatically produced breakdown product of lipid peroxidation, also opens mitoK $\mathrm{ATP}_{\mathrm{TP}}$ via $\mathrm{PKC} \varepsilon$, but it does so at about 10 -fold lower potency than $\mathrm{FAOOH}$ (data not shown). In addition, nitration of unsaturated fatty acids yields electrophilic nitroalkene derivatives $\left(\mathrm{FANO}_{2}\right)$ known to function as pluripotent endogenous signaling molecules (67) and nitroalkenes were shown to be formed

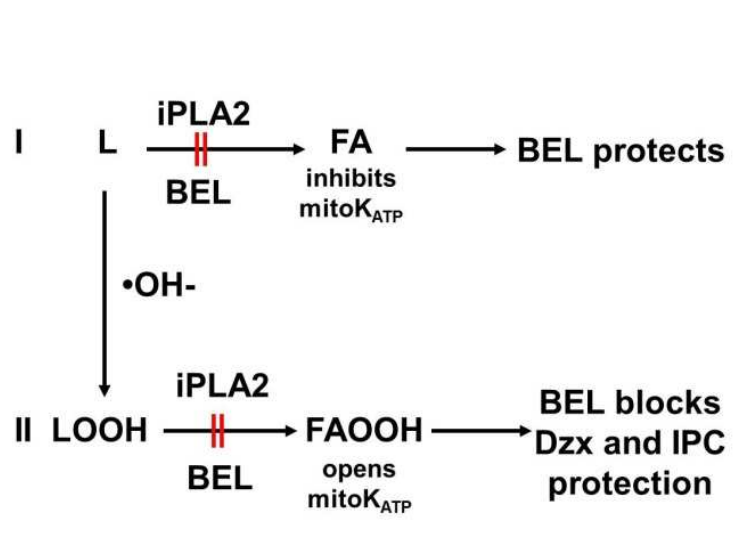

Fig. 3.8. Dual role of iPLA2 action in mitochondria and cardioprotection. (I) When a fatty acid side chain (L) interacts with the $\mathrm{Ca}^{2+}$ independent phospholipase A2 (iPLA2), free fatty acids (FA) are formed, which inhibit mitoK $_{\text {ATP }}$ and cause damage to the untreated heart. In this case, bromoenol lactone (BEL), the IPLA2 inhibitor, acts as a protective agent because it blocks the formation of FA and allows mitoK $\mathrm{K}_{\mathrm{ATP}}$ opening to occur. (II) When iPLA2 interacts with a lipid hydroperoxide, hydroperoxy fatty acids (FAOOH) are formed, which open mitoK ${ }_{\text {ATP }}$ and confer protection. In this case, BEL blocks both diazoxide and IPC protection because the formation of the proposed signaling ROS is blocked. in mitochondria during IPC (105).

Therefore, we have also tested the effect of nitro oleic acid $\left(\mathrm{OANO}_{2}\right)$ on mitoK $_{\text {ATP. }} 1 \mu \mathrm{M}$ nitro oleic acid exhibited properties similar to the tested linoleic acid hydroperoxide.

Namely, the $\mathrm{OANO}_{2}$ had no effect on the pharmacologically opened

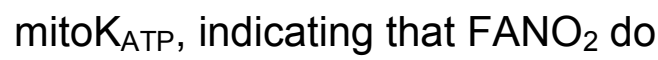
not inhibit mitoK $\mathrm{K}_{\text {ATP. }}$. However, $\mathrm{OANO}_{2}$ was able to reverse the inhibitory effect of ATP to the level of control open trace and this opening effect was inhibited by $5-\mathrm{HD}$, indicating that the effect $\mathrm{OANO}_{2}$ was mediated by mitoK $\mathrm{K}_{\mathrm{ATP}}$. The main difference between the opening effects of $\mathrm{LAOOH}$ and $\mathrm{OANO}_{2}$ is supported by the data showing that the effect of $\mathrm{OANO}_{2}$ is not 
sensitive to the specific inhibitor peptide $\varepsilon \mathrm{V}_{1-2}$, indicating that the effect of $\mathrm{OANO}_{2}$ acts independently of PKC $\varepsilon$.

To determine the effect of fatty acids on mitoK $\mathrm{K}_{\mathrm{ATP}}$ under the conditions of endogenous ROS production by mitochondria, we utilized a previously established preincubation protocol. This triggers a feedback activation mechanism that keeps mitoK $\mathrm{K}_{\mathrm{ATP}}$ in its open state by increasing ROS production at Complex I of the respiratory chain (2) in a PKC $\varepsilon$-dependent manner (20). The endogenous open state of mitoK $\mathrm{K}_{\mathrm{ATP}}$ was prevented by both LA and 13-(S)-HODE, which were tested at a concentration sufficient to fully inhibit the pharmacologically open mitoK $\mathrm{K}_{\text {ATP. }}$ In addition, the phosphorylated open state was blocked by racemic bromoenol lactone (BEL), the inhibitor of phospholipases iPLA2 (8). The inhibition of the ROS-induced open state by BEL implies the participation of the mitochondrial phospholipases iPLA2 in the regulation of mitoK $_{\text {ATP. }}$ These experiments indicate that a compound responsible for the ROSinduced activation of PKC $\varepsilon$ is dependent upon the enzymatic function of iPLA2.

FAOOH arise from peroxidation of polyunsaturated fatty acids (PUFA) derived from membrane phospholipids or alternatively from the enzymatic action of lipoxygenases (LOX). As shown in Fig. 3.8, both mechanisms require iPLA2. In the following sets of experiments, we tested the hypothesis that iPLA2 activity is essential for intramitochondrial signaling and for cardioprotection. In order to 

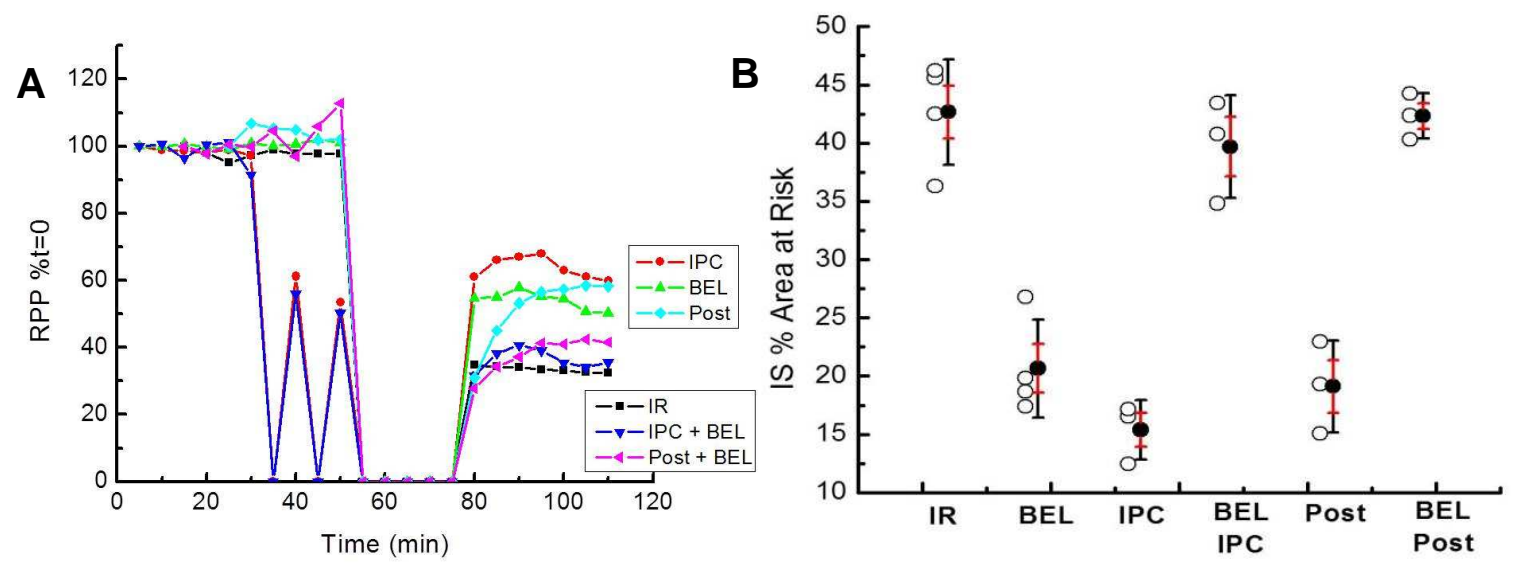

Fig. 3.9. Inhibition of Ca2+-independent phospholipase A2 (iPLA2) demonstrates that FAOOH are necessary for signaling. (A) BEL is shown here to block IPC ("IPC+BEL") and ischemic postconditioning ("Post+BEL") improvements in functional recovery but also to confer protection when administered alone ("BEL"). Data representative of at least 3 independent experiments. (B) Infarct size was significantly reduced with the administration of IPC and postconditioning and this was matched by treatment with BEL alone. However, BEL inhibited the protective effects of both IPC and postconditioning. Data are means $\pm S D$, representative of at least 3 independent experiments. Not shown is BEL's ability to block diazoxide as well.

approach the hypotheses, the ability of the iPLA2 inhibitor bromoenol lactone (BEL) to block cardioprotection was tested in the perfused heart. Fig. 3.9 shows that BEL protects the heart and reduces infarct size when added alone, consistent with previously published findings (149). These results are consistent with BEL protecting against ischemic reperfusion injury by preventing ischemic release of free fatty acids, which are known to accumulate during myocardial ischemia $(81,142)$. However, BEL blocks protection by both ischemic preconditioning (IPC) and postconditioning (PC) when added with either treatment. We interpret these results as the ability of BEL to prevent the iPLA2dependent release of $\mathrm{FAOOH}$, which are necessary for protection. 


\section{3-E: Discussion}

Fatty acids are the preferred metabolic substrates of the heart, but when elevated they are harmful to the heart. Mitochondria play a key role in both ischemia-reperfusion injury (IRI) and cardioprotection and the opening of mitoK $\mathrm{K}_{\mathrm{ATP}}$ was shown to be obligatory in all tested protocols of cardioprotection. 5$\mathrm{HD}$ is a simple hydroxy fatty acid which is frequently used in many studies as the specific inhibitor of mitoK $\mathrm{ATP}_{\mathrm{T}}(125)$. Here we studied whether other fatty acids have a similar ability to inhibit the pharmacologically opened mitoK $\mathrm{K}_{\mathrm{ATP}}$. We focused on linoleic acid and its metabolites in this study because these are the detected products of mitochondrial cardiolipin cleavage, which, during ischemia, suffers the most profound change (112). Linoleic acid inhibited the pharmacological open state of mitoK $\mathrm{ATP}_{\text {in }}$ in the presence of ATP and diazoxide. The half-maximal inhibition observed under the given experimental condition yielded a mean value of $0.46 \mu \mathrm{M}$. This value is based on total fatty acid added to the assay and will vary with experimental conditions, primarily with the concentration of phospholipids in the assay. The corresponding value in free fatty acid concentration can be calculated based on the known extinction coefficient for linoleic acid and the calculation based on our experimental conditions yields an approximate value of 250 nM. Our data in Fig. 3.2 further show that not only linoleic acid, but the hydroxy derivative 13-hydroxydecadenoic acid (13-(S)HODE), a stable product of linoleic acid peroxidation, also inhibits mitoK ATP. $_{\text {. }}$ These data indicate that natural fatty acids and their hydroxy derivatives behave similar to $5-\mathrm{HD}$ and inhibit the pharmacologically open state of mitoK $\mathrm{ATP}_{\text {TP. }}$ 
FA become elevated in two distinct ways: in association with obesity and/or metabolic syndrome, and in association with ischemia itself, due to activation of phospholipases. Pirro, et al (117) showed that elevated plasma FA concentrations are associated with an increased risk of ischemic heart disease, and Lopaschuk et al (92) noted that the risk for cardiovascular disease such as myocardial infarction and heart failure is drastically increased with obesity. Obesity has also been shown to be harmful experimentally. IPC failed to protect leptin-deficient ob/ob mice (11), and both IPC and diazoxide failed to protect Zucker obese rats (77). These studies, however, do not address the question as to whether FA have acute effects on cardioprotection, because compensatory adaptations may have occurred in man or genetically altered mice. In an important study, Ford, et al (33) found that the working rat heart was no longer protected from ischemia-reperfusion injury by cromakalim when the perfusate included $1.2 \mathrm{mM}$ palmitate (as well as glucose and insulin). Our data shown in Fig. 3.5 support the view that elevated FA directly and acutely interfere with cardioprotection. Fatty acids, including linoleic acid and its hydroxy derivative, 13-(S)-HODE, acutely block protection by IPC and diazoxide.

Our previous results have established hydroxyl radicals $\left(\mathrm{HO}^{\circ}\right)$ as a key component of cardioprotective signaling (36). Hydroxyl radicals readily oxidize the side chains of polyunsaturated phospholipids, rendering them more susceptible to cleavage by phospholipases and resulting in formation of hydroperoxy fatty acids, FAOOH (Reaction II, Fig. 3.8). FAOOH are transient, non-radical and highly reactive species (72) and are known to activate protein 
kinases (134). We also showed some years ago that FAOOH can readily flip-flop across the inner membrane bilayer (72) and thus reach its receptor on the other side of the membrane.

Fig. 3.6 contains results showing that hydroperoxy linoleic acid ( $\mathrm{LAOOH})$ is a potent mitoK $\mathrm{ATP}_{\text {P }}$ opener. Fig. $\mathbf{3 . 6}$ also shows that $\mathrm{LAOOH}$ acts via $\mathrm{PKC} \varepsilon$, because its effect is blocked by the specific peptide $\varepsilon \mathrm{V}_{1-2}$. $\mathrm{LAOOH}$ is the expected product of non-enzymatic peroxidation due to the action of hydroxyl radical on the side chains of polyunsaturated phospholipids, which are represented in mitochondria mainly by cardiolipin (88). Alternatively, LAOOH is a product of enzymatic peroxidation of linoleic acid cleaved from cardiolipin due to the action of lipoxygenases (Reaction III, Fig. 3.8). The role of lipoxygenase metabolites in ischemic preconditioning was studied previously with the focus on leukocyte type 12-lipoxygenase (12-LOX) metabolites of arachidonic acid (16, 100). The data suggested that 12-LOX metabolism plays an important role in cardioprotection. The results, however, did not address the role of mitochondria and also could not distinguish between the actions of hydroperoxy fatty acids and the subsequently produced hydroxy fatty acids, as only the corresponding elevated levels of hydroxy fatty acid analogue of arachidonic acid were measured as the stable metabolite indicating the actions of activated 12-LOX. Nevertheless, 12-LOX deficient mice show impaired ischemic preconditioning-induced cardioprotection (35). In our view, these results indicate the intriguing possibility that 12-LOX is co-localized to mitochondria, a hypothesis which awaits further 
investigation. In support of the view that $\mathrm{FAOOH}$ are cardioprotective, we show in Fig. 3.7 that exogenous $\mathrm{LAOOH}$ confers cardioprotection in isolated perfused rat heart comparable to the protection achieved by diazoxide.

Ischemic preconditioning is also known to yield elevated levels of nitric oxide with consequent formation of nitroalkenes, such as nitro fatty acids (105). We have therefore also investigated whether nitro oleic acid $\left(\mathrm{OANO}_{2}\right)$ acts in a manner similar to $\mathrm{LAOOH}$ and activates mitoK $\mathrm{ATP}_{\mathrm{A}}$ via $\mathrm{PKC} \varepsilon$ - dependent pathway. $\mathrm{OANO}_{2}$ induces 5-HD-sensitive opening of ATP-inhibited mitoK ${ }_{\text {ATP }}$ but it is not sensitive to the specific $P K C \varepsilon$ inhibitor $\varepsilon \bigvee_{1-2}$, indicating that the action of $\mathrm{OANO}_{2}$ is $\mathrm{PKC} \varepsilon$-independent. This finding agrees with recent observations that mitoK $\mathrm{K}_{\mathrm{ATP}}$ was activated by nitrolinoleate $\left(\mathrm{LANO}_{2}\right)(119)$ and also with the finding that the cardioprotective effects of $\mathrm{LANO}_{2}$ were not attributable to protective kinase signaling (105).

If hydroperoxy fatty acids (FAOOH) are the ROS species essential for intramitochondrial signaling, then it follows that phospholipase iPLA2 is essential for cardioprotection. iPLA2 was shown to be localized to mitochondria (13, 93)and also to be activated by elevated exogenous ROS in isolated mitochondria (74). The enzymatic actions of iPLA2 lead to release of either the FAOOH from the oxidized phospholipid side chains, or to release of FA that are oxidized to $\mathrm{FAOOH}$ by the enzymatic action of lipoxygenase, enabling $\mathrm{FAOOH}$ to diffuse to their $\mathrm{PKC} \varepsilon$ targets. Our following new findings summarized in Fig. 3.9 support this hypothesis: 1) bromoenol lactone (BEL) blocks PKC $\varepsilon$-dependent mitoK $\mathrm{ATP}_{\mathrm{AT}}$ 
opening; and 2) BEL blocks protection by IPC and ischemic postconditioning. Note that BEL protects the unconditioned heart as shown previously by Williams and Gottlieb (149). This occurs because BEL blocks ischemic release of FAs that inhibit mitoK $\mathrm{K}_{\text {ATP. }}$ This dual role of IPLA2 has not previously been described and is due to the fact that iPLA2 acts on both un-oxidized and oxidized phospholipids.

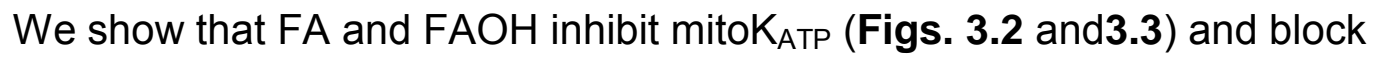
protection (Fig. 3.5). FAOOH released from oxidized lipids or formed by the enzymatic action of lipoxygenases are potent activators of the mitochondrial

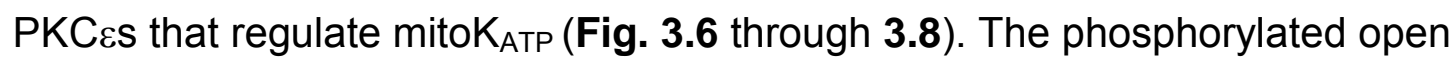
state of mitoK $\mathrm{K}_{\text {ATP }}$ is less sensitive to the inhibitory effect of FA, as demonstrated by the preincubation experiments involving LA and 13-(S)-HODE. The BEL experiments shown in Fig. $\mathbf{3 . 9}$ establish that products of lipid peroxidation and cleavage provide the ROS signal and that $\mathrm{FAOOH}$ or one of its products is the ROS signal. It leads to the further hypothesis that mitochondrial PLA2 is an essential component of cardioprotection.

\section{Acknowledgments}

The authors express gratitude for the technical and administrative assistance of Craig Semrad and Beau Sober. This work was supported by grants HL35673 and HL067842 from the National Heart, Lung and Blood Institute to K.D. Garlid, and by grant ME09018 to M. Jaburek. 


\section{Chapter 4: Conclusions}

The primary aim of this project is to identify the reactive oxygen species (ROS) that acts as the second messenger in cardioprotective signaling by ischemic and pharmacological preconditioning. The results of this study lead to two conclusions: 1) neither hydrogen peroxide $\left(\mathrm{H}_{2} \mathrm{O}_{2}\right)$ nor hydroxyl radical $\left(\mathrm{HO} \mathrm{O}^{\circ}\right)$ is responsible for feedback activation of mitoK $_{\mathrm{ATP}}$ and 2) the signaling ROS is a downstream oxidation product of $\mathrm{HO}^{\circ}$, namely a hydroperoxy fatty acid (FAOOH) released by the calcium-independent phospholipase A2 (iPLA2). These results further the understanding of signaling in cardioprotection and dispel widely-held misunderstandings about the nature and identity of ROS signaling molecules. Furthermore, signaling ROS are crucial to processes across cell types and this research provides a location of ROS signal formation, a model for their transformation and a likely downstream candidate to seek in other systems.

$\mathrm{H}_{2} \mathrm{O}_{2}$ and $\mathrm{HO}^{\circ}$ are products of oxidation-reduction reactions beginning with superoxide $\left(\mathrm{O}_{2}{ }^{\circ-}\right)$ generated at complex I of the electron transport chain. Brookes et al. claimed that $\mathrm{O}_{2}{ }^{-}$is the signaling ROS but failed to take into account the production of $\mathrm{H}_{2} \mathrm{O}_{2}$ (119). By adding catalase to their media as a control, Costa et al. demonstrated that $\mathrm{O}_{2}{ }^{*-}$ does not directly activate mitoK $\mathrm{ATP}_{\text {but }}$ but $\mathrm{H}_{2} \mathrm{O}_{2}$ is responsible for the mitoK $\mathrm{ATP}_{\text {ATP }}$ effect in Brookes' system (20). Schumacker et al. proposed that $\mathrm{H}_{2} \mathrm{O}_{2}$ is the signaling ROS and this was widely accepted without question $(86,145)$. However, the matrix of heart mitochondria contains large amounts of catalase and other antioxidants (121), which would likely consume the small amounts of $\mathrm{H}_{2} \mathrm{O}_{2}$ produced (132), indicating that $\mathrm{H}_{2} \mathrm{O}_{2}$ may not be a 
viable signaling ROS in cardioprotection. Indeed, the data presented here demonstrate that $\mathrm{H}_{2} \mathrm{O}_{2}$ is not the signaling ROS, namely due to the lack of an effect by catalase on the feedback-activated state of mitoK $\mathrm{K}_{\text {ATP }}$ and the collection of scavengers and chain-breaking antioxidants indicating a signal downstream of $\mathrm{HO}^{\circ}(36)$.

Downey et al. proposed $\mathrm{HO}^{\circ}$ as the signaling ROS (6), but it is far too short lived to serve as a transportable signaling species (87). Furthermore, this conclusion was based on experiments utilizing MPG, a purported $\mathrm{HO}^{\circ}$-scavenger with strong thiol-reductant properties that confound experiments on mitoK $\mathrm{K}_{\mathrm{ATP}}$ due to its effect on PKCE. The $\mathrm{HO}^{\circ}$-scavenging capabilities of MPG cannot be tested here because mitoK $\mathrm{ATP}_{\mathrm{A}}$ will be inhibited whether or not $\mathrm{HO} \mathrm{O}^{\circ}$ is present with MPG keeping $\mathrm{PKC} \varepsilon$ in its reduced state. Accordingly, our data indicate that the signaling ROS is a downstream oxidation product of $\mathrm{HO}^{\circ}$ that acts as the second messenger in cardioprotective signaling and we have identified this ROS as a hydroperoxy fatty acid.

We have demonstrated here that $\mathrm{FAOOH}$ are responsible for feedback activation of mitoK $\mathrm{K}_{\mathrm{ATP}}$ and MPT inhibition and that they are necessary in cardioprotection by ischemic and pharmacological preconditioning. Fatty acid side chains are peroxidized by the ROS generated from mitoK $\mathrm{ATP}_{\text {opening }}$ and $\mathrm{FAOOH}$ are released by the action of iPLA2 on these peroxidized side chains. FAOOH are then able to flip-flop across membranes (76) and could serve as a transportable form of ROS for a variety of signaling processes. Further studies 
exploring the life-time and transportability of $\mathrm{FAOOH}$ as well as their role in other signaling processes are needed to complete this project and gain a greater understanding of ROS signaling from the mitochondrion.

While this study shows that $\mathrm{FAOOH}$ are a protective ROS, their nonperoxidized counterparts, fatty acids (FA), have been demonstrated to present dangers in a number of ways. FA become elevated in association with obesity and during ischemia itself by the action of phospholipases that release FA from phospholipid side chains. Elevated plasma FA is associated with a greater risk of ischemic heart disease (117) and the risk for cardiovascular disease such as myocardial infarction and heart failure is drastically increased with obesity (92). There are many avenues to pursue for therapeutic and preventative treatments of these conditions, but an in-depth understanding of the signaling processes involved is certainly necessary.

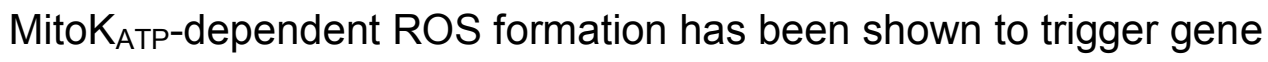
transcription (46)and is also necessary in endogenous cardioprotective signaling via bradykinin, opioid and adenosine $G_{i}$ protein-coupled receptor (GPCR) pathways (29). GPCR signaling has recently been found to operate via a 'signalosome', a unique signaling model that transports the receptor-agonist complex formed at the cell membrane along with the entire signaling cascade in a caveolar raft (120). These signalosomes likely travel along microtubules as opposed to relying on diffusion, and inhibitors of microtubules have been shown to block preconditioning (106). ROS is required for activation of the adenosine 
signalosome and it is also necessary for the feedback activation that establishes cardioprotective 'memory' witnessed in preconditioning $(20,144)$.

ROS signaling has been invoked by research labs across nearly all fields. Notably, Muscari et al. recently published a paper involving hypoxic pretreatment of adult stem cells for the purpose of developing more effective regenerative medicines by accomplishing greater survival rates for grafted stem cells (104). While multipotent stem cells could be of great use for in-vivo tissue repair, they suffer from a poor post-engraftment survival rate that makes the clinical treatment unviable. Ischemic preconditioning (IPC) certainly has the potential to serve as an important treatment in improving stem-cell survival and signaling ROS play a vital role in this process. As stated in the review, "mitochondrial ROS are consistently involved in the causative mechanisms of IPC" (104) and identifying the species behind these mechanisms is necessary for a full understanding of protective signaling.

The pragmatic value or application of this research rests in the future development of therapeutic and preventative treatment against heart disease and the damage resulting from heart attacks. While an immediate benefit from these studies are unavailable, uncovering the pathways and crucial players in signaling pathways of cardioprotection is vital to our future understanding of and ability to treat these diseases. Furthermore, the signaling processes at play in cardioprotection are by no means unique to the heart; mitoK $\mathrm{ATP}_{\mathrm{T}}$ and ROS signaling play integral roles in signaling throughout all cell types. 
With so much of the population at risk of heart disease, heart attacks, diabetes, obesity and a variety of mitochondrial diseases, it is of utmost importance that we explore these questions and undertake the efforts in basic research necessary to lay the foundation for treatment and prevention. 


\section{References}

1. Ammala C, Moorhouse A, and Ashcroft FM. The sulphonylurea receptor confers diazoxide sensitivity on the inwardly rectifying $\mathrm{K}+$ channel Kir6.1 expressed in human embryonic kidney cells. J Physiol 494: 709-714., 1996. 2. Andrukhiv A, Costa AD, West IC, and Garlid KD. Opening mitoK ATP $_{\text {. }}$ increases superoxide generation from Complex I of the electron transport chain. Am J Physiol 291: H2067-2074, 2006.

3. Argaud L, Gateau-Roesch O, Raisky O, Loufouat J, Robert D, and Ovize M. Postconditioning inhibits mitochondrial permeability transition. Circulation 111: 194-197, 2005.

4. Argaud L, Loufouat J, Gateau-Roesch O, Gomez L, Robert D, and Ovize M. Persistent Inhibition of Mitochondrial Permeability Transition by Preconditioning During the First Hours of Reperfusion. Shock 28: 28, 2008. 5. Babbs CF, and Steiner MG. Detection and quantitation of hydroxyl radical using dimethyl sulfoxide as molecular probe. Methods Enzymol 186: 137147, 1990.

6. Baines CP, Goto M, and Downey JM. Oxygen radicals released during ischemic preconditioning contribute to cardioprotection in the rabbit myocardium. J Mol Cell Cardiol 29: 207-216, 1997.

7. Balafanova Z, Bolli R, Zhang J, Zheng Y, Pass JM, Bhatnagar A, Tang $X L$, Wang O, Cardwell E, and Ping P. Nitric oxide (NO) induces nitration of protein kinase Cepsilon (PKCepsilon), facilitating PKCepsilon translocation via enhanced PKCepsilon -RACK2 interactions: a novel mechanism of no-triggered activation of PKCepsilon. J Biol Chem 277: 15021-15027, 2002.

8. Balsinde J, Bianco ID, Ackermann EJ, Conde-Frieboes K, and Dennis EA. Inhibition of calcium-independent phospholipase A2 prevents arachidonic acid incorporation and phospholipid remodeling in P388D1 macrophages. Proc Natl Acad Sci U S A 92: 8527-8531, 1995.

9. Beavis AD, Brannan RD, and Garlid KD. Swelling and contraction of the mitochondrial matrix. I. A structural interpretation of the relationship between light scattering and matrix volume. J Biol Chem 260: 13424-13433, 1985.

10. Bolli R, Jeroudi MO, Patel BS, Aruoma OI, Halliwell B, Lai EK, and McCay PB. Marked reduction of free radical generation and contractile dysfunction by antioxidant therapy begun at the time of reperfusion. Evidence that myocardial "stunning" is a manifestation of reperfusion injury. Circ Res 65: 607-622, 1989.

11. Bouhidel O, Pons S, Souktani R, Zini R, Berdeaux A, and Ghaleh B. Myocardial ischemic postconditioning against ischemia-reperfusion is impaired in ob/ob mice. Am J Physiol 295: H1580-1586, 2008.

12. Cadenas E, and Davies KJ. Mitochondrial free radical generation, oxidative stress, and aging. Free Radic Biol Med 29: 222-230, 2000.

13. Cedars A, Jenkins CM, Mancuso DJ, and Gross RW. Calciumindependent phospholipases in the heart: mediators of cellular signaling, bioenergetics, and ischemia-induced electrophysiologic dysfunction. $J$ Cardiovasc Pharmacol 53: 277-289, 2009. 
14. Chakraborty S, Berwick ZC, Bartlett PJ, Kumar S, Thomas AP, Sturek M, Tune JD, and Obukhov AG. Bromoenol lactone inhibits voltage-gated Ca2+ and transient receptor potential canonical channels. J Pharmacol Exp Ther 339: 329-340, 2011.

15. Chen W, Gabel S, Steenbergen C, and Murphy E. A redox-based mechanism for cardioprotection induced by ischemic preconditioning in perfused rat heart. Circ Res 77: 424-429, 1995.

16. Chen W, Glasgow W, Murphy E, and Steenbergen C. Lipoxygenase metabolism of arachidonic acid in ischemic preconditioning and PKC-induced protection in heart. Am J Physiol 276: H2094-2101, 1999.

17. Costa AD, Garlid KD, West IC, Lincoln TM, Downey JM, Cohen MV, and Critz SD. Protein Kinase G Transmits the Cardioprotective Signal From Cytosol to Mitochondria. Circ Res 97: 329-336, 2005.

18. Costa AD, Jakob R, Costa CL, Andrukhiv K, West IC, and Garlid KD. The mechanism by which mitoK $\mathrm{ATP}_{\text {P }}$ opening and $\mathrm{H}_{2} \mathrm{O}_{2}$ inhibit the mitochondrial permeability transition. J Biol Chem 281: 20801-20808, 2006.

19. Costa AD, Pierre SV, Cohen MV, Downey JM, and Garlid KD. cGMP Signaling in Pre- and Postconditioining: The Role of Mitochondria. Cardiovasc Res 77: 344-352, 2008.

20. Costa ADT, and Garlid KD. Intramitochondrial signaling: interactions among mitoK ATP $_{\text {PKC }}$, ROS, and MPT. Am J Physiol 295: H874-882, 2008. 21. Costa ADT, Quinlan C, Andrukhiv A, West IC, and Garlid KD. The direct physiological effects of mitoK $\mathrm{K}_{\mathrm{ATP}}$ opening on heart mitochondria. $\mathrm{Am} \mathrm{J}$ Physiol 290: H406-415, 2006.

22. Crompton $\mathbf{M}$. The mitochondrial permeability transition pore and its role in cell death. Biochem J 341: 233-249, 1999.

23. Das DK, Engelman RM, and Maulik N. Oxygen free radical signaling in ischemic preconditioning. Annals Of The New York Academy Of Sciences 874: 49-65, 1999.

24. Di Lisa F, and Bernardi P. Mitochondria and ischemia-reperfusion injury of the heart: fixing a hole. Cardiovasc Res 70: 191-199, 2006.

25. Di Lisa F, Menabo R, Canton M, Barile M, and Bernardi P. Opening of the mitochondrial permeability transition pore causes depletion of mitochondrial and cytosolic $\mathrm{NAD}^{+}$and is a causative event in the death of myocytes in postischemic reperfusion of the heart. J Biol Chem 276: 2571-2575, 2001.

26. Diwan JJ, Haley T, and Sanadi DR. Reconstitution of transmembrane K+ transport with a 53 kilodalton mitochondrial protein. Biochem Biophys Res Commun 153: 224-230, 1988.

27. Dix TA, and Aikens $\mathbf{J}$. Mechanisms and biological relevance of lipid peroxidation initiation. Chem Res Toxicol 6: 2-18, 1993.

28. Dos Santos P, Kowaltowski AJ, Laclau MN, Seetharaman S, Paucek P, Boudina S, Thambo JB, Tariosse L, and Garlid KD. Mechanisms by which opening the mitochondrial ATP- sensitive $\mathrm{K}^{+}$channel protects the ischemic heart. Am J Physiol 283: H284-295., 2002. 
29. Downey JM, Davis AM, and Cohen MV. Signaling pathways in ischemic preconditioning. Heart Fail Rev 12: 181-188, 2007.

30. Droge W. Free radicals in the physiological control of cell function. Physiol Rev 82: 47-95., 2002.

31. Duranteau J, Chandel NS, Kulisz A, Shao Z, and Schumacker PT. Intracellular signaling by reactive oxygen species during hypoxia in cardiomyocytes. J Biol Chem 273: 11619-11624, 1998.

32. Forbes RA, Steenbergen C, and Murphy E. Diazoxide-Induced Cardioprotection Requires Signaling Through a Redox-Sensitive Mechanism. Circ Res 88: 802-809, 2001.

33. Ford WR, Lopaschuk GD, Schulz R, and Clanachan AS. K(ATP)channel activation: effects on myocardial recovery from ischaemia and role in the cardioprotective response to adenosine A1-receptor stimulation. Br J Pharmacol 124: 639-646, 1998.

34. Foster DB, Ho AS, Rucker J, Garlid AO, Chen L, Sidor A, Garlid KD, and O'Rourke B. Mitochondrial ROMK channel is a molecular component of mitoK(ATP). Circ Res 111: 446-454, 2012.

35. Gabel SA, London RE, Funk CD, Steenbergen C, and Murphy E. Leukocyte-type 12-lipoxygenase-deficient mice show impaired ischemic preconditioning-induced cardioprotection. Am J Physiol 280: H1963-1969, 2001. 36. Garlid AO, Jaburek M, Jacobs JP, and Garlid KD. Mitochondrial Reactive Oxygen Species - Which ROS Signals Cardioprotection? Am J Physiol Heart Circ Physiol 2013.

37. Garlid KD. Cation transport in mitochondria--the potassium cycle. Biochim Biophys Acta 1275: 123-126, 1996.

38. Garlid KD. Chemiosmotic Theory. In: Encylopedia Biol Chem, edited by Carafoli E. Amsterdam: Elsevier, 2004, p. (in press).

39. Garlid KD. On the mechanism of regulation of the mitochondrial $\mathrm{K}^{+} / \mathrm{H}^{+}$ exchanger. J Biol Chem 255: 11273-11279, 1980.

40. Garlid KD. Opening mitochondrial $\mathrm{K}_{\mathrm{ATP}}$ in the heart--what happens, and what does not happen. Basic Res Cardiol 95: 275-279., 2000.

41. Garlid KD. The state of water in biological systems. Int Rev Cytol 192: 281-302., 2000.

42. Garlid KD. Unmasking the mitochondrial K/H exchanger: swelling-induced $\mathrm{K}^{+}$-loss. Biochem Biophys Res Commun 83: 1450-1455, 1978.

43. Garlid KD, and Beavis AD. Swelling and contraction of the mitochondrial matrix. II. Quantitative application of the light scattering technique to solute transport across the inner membrane. J Biol Chem 260: 13434-13441, 1985.

44. Garlid KD, Beavis AD, and Ratkje SK. On the nature of ion leaks in energy-transducing membranes. Biochim Biophys Acta 976: 109-120, 1989. 45. Garlid KD, Costa ADT, Quinlan CL, Pierre SV, and Dos Santos P. Cardioprotective Signaling to Mitochondria. J Mol Cell Cardiol 46: 858-866, 2009. 46. Garlid KD, Dos Santos P, Xie Z-J, Costa ADT, and Paucek P. Mitochondrial potassium transport: the role of the mitochondrial ATP-sensitive $\mathrm{K}^{+}$ 
channel in cardiac function and cardioprotection. Biochim Biophys Acta 1606: 121, 2003.

47. Garlid KD, and Halestrap AP. The mitochondrial K(ATP) channel--fact or fiction? J Mol Cell Cardiol 52: 578-583, 2012.

48. Garlid KD, and Paucek P. Mitochondrial potassium transport: the $\mathrm{K}^{+}$ cycle. Biochim Biophys Acta 1606: 23-41, 2003.

49. Garlid KD, Paucek P, Yarov-Yarovoy V, Murray HN, Darbenzio RB, D'Alonzo AJ, Lodge NJ, Smith MA, and Grover GJ. Cardioprotective effect of diazoxide and its interaction with mitochondrial ATP-sensitive $\mathrm{K}^{+}$channels. Possible mechanism of cardioprotection. Circ Res 81: 1072-1082, 1997.

50. Garlid KD, Paucek P, Yarov-Yarovoy V, Sun X, and Schindler PA. The mitochondrial $\mathrm{K}_{\text {ATP }}$ channel as a receptor for potassium channel openers. J Biol Chem 271: 8796-8799, 1996.

51. Garlid KD, Puddu PE, Pasdois P, Costa ADT, Beauvoit B, Criniti A, Tariosse L, Diolez $\mathbf{P}$, and Dos Santos $\mathbf{P}$. Inhibition of cardiac contractility by 5hydroxydecanoate and tetraphenylphosphonium ion: a possible role of mitoK ATP $_{\text {A }}$ in the response to inotropic stress. Am J Physiol 291: H152-160, 2006.

52. Garlid KD, Sun X, Paucek $\mathbf{P}$, and Woldegiorgis $\mathbf{G}$. Mitochondrial cation transport systems. Methods Enzymol 260: 331-348, 1995.

53. Gateau-Roesch O, Argaud L, and Ovize M. Mitochondrial permeability transition pore and postconditioning. Cardiovasc Res 70: 264-273, 2006.

54. Gornall AG, Bardawill CJ, and David MM. Determination of serum proteins by means of the biuret reaction. J Biol Chem 177: 751-766, 1949.

55. Goto M, Liu Y, Yang XM, Ardell JL, Cohen MV, and Downey JM. Role of bradykinin in protection of ischemic preconditioning in rabbit hearts. Circ Res 77: 611-621, 1995.

56. Gross GJ, Warltier DC, and Hardman HF. Comparative effects of nicorandil, a nicotinamide nitrate derivative, and nifedipine on myocardial reperfusion injury in dogs. J Cardiovasc Pharmacol 10: 535-542, 1987.

57. Grover GJ. Protective effects of ATP-sensitive potassium-channel openers in experimental myocardial ischemia. J Cardiovasc Pharmacol 24 Suppl 4: S18-27, 1994.

58. Grover GJ, D'Alonzo AJ, Hess T, Sleph PG, and Darbenzio RB. Glyburide-reversible cardioprotective effect of BMS-180448 is independent of action potential shortening. Cardiovasc Res 30: 731-738, 1995.

59. Grover GJ, D'Alonzo AJ, Parham CS, and Darbenzio RB.

Cardioprotection with the KATP opener cromakalim is not correlated with ischemic myocardial action potential duration. J Cardiovasc Pharmacol 26: 145152, 1995.

60. Grover GJ, and Garlid KD. ATP-Sensitive potassium channels: a review of their cardioprotective pharmacology. J Mol Cell Cardiol 32: 677-695., 2000.

61. Grover GJ, McCullough JR, Henry DE, Conder ML, and Sleph PG.

Anti-ischemic effects of the potassium channel activators pinacidil and cromakalim and the reversal of these effects with the potassium channel blocker glyburide. J Pharmacol Exp Ther 251: 98-104, 1989. 
62. Gui G, Hegazy MG, Mironova G, Mahdi F, Beavis A, and Garlid KD. Purification and reconstitution of the mitochondrial K+ channel. J Mol Cell Cardiol 23: S78, 1991.

63. Halliwell B, and Gutteridge JMC. Free radicals in biology and medicine. New York: Oxford University Pess, 2007.

64. Harris L, Kimura Y, and Shaikh NA. Phospholipase inhibition and the electrophysiology of acute ischemia: studies with amiodarone. J Mol Cell Cardiol 25: 1075-1090, 1993.

65. Hausenloy DJ, Maddock HL, Baxter GF, and Yellon DM. Inhibiting mitochondrial permeability transition pore opening: a new paradigm for myocardial preconditioning? Cardiovasc Res 55: 534-543, 2002.

66. Hegazy MG, Mahdi F, Li X, Gui G, Mironova G, Beavis AD, and Garlid KD. Purification and reconstitution of the rat liver mitochondrial $K+$ uniporter. Biophys J 59: A136, 1991.

67. Iles KE, Wright MM, Cole MP, Welty NE, Ware LB, Matthay MA, Schopfer FJ, Baker PR, Agarwal A, and Freeman BA. Fatty acid transduction of nitric oxide signaling: nitrolinoleic acid mediates protective effects through regulation of the ERK pathway. Free Radic Biol Med 46: 866-875, 2009. 68. Imai H, and Nakagawa Y. Biological significance of phospholipid hydroperoxide glutathione peroxidase (PHGPx, GPx4) in mammalian cells. Free Radic Biol Med 34: 145-169, 2003.

69. Inagaki N, Gonoi T, Clement JPt, Namba N, Inazawa J, Gonzalez G, Aguilar-Bryan L, Seino S, and Bryan J. Reconstitution of IKATP: an inward rectifier subunit plus the sulfonylurea receptor [see comments]. Science 270: 1166-1170, 1995.

70. Inoue I, Nagase H, Kishi K, and Higuti T. ATP-sensitive K+ channel in the mitochondrial inner membrane. Nature 352: 244-247, 1991.

71. Jaburek M, Costa ADT, Burton JR, Costa CL, and Garlid KD.

Mitochondrial PKCepsilon and mitoK $\mathrm{K}_{\text {ATP }}$ co-purify and co-reconstitute to form a functioning signaling module in proteoliposomes. Circ Res 99: 878-883, 2006.

72. Jaburek M, Miyamoto S, Di Mascio P, Garlid KD, and Jezek P. Hydroperoxy fatty acid cycling mediated by mitochondrial uncoupling protein UCP2. J Biol Chem 279: 53097-53102, 2004.

73. Jenkins CM, Cedars A, and Gross RW. Eicosanoid signalling pathways in the heart. Cardiovasc Res 82: 240-249, 2009.

74. Jezek J, Jaburek M, Zelenka J, and Jezek P. Mitochondrial phospholipase A2 activated by reactive oxygen species in heart mitochondria induces mild uncoupling. Physiol Res 59: 737-747, 2010.

75. Jezek $\mathbf{P}$, and Hlavata $\mathbf{L}$. Mitochondria in homeostasis of reactive oxygen species in cell, tissues, and organism. Int J Biochem Cell Biol 37: 2478-2503, 2005.

76. Kamp F, Zakim D, Zhang F, Noy N, and Hamilton JA. Fatty acid flip-flop in phospholipid bilayers is extremely fast. Biochemistry 34: 11928-11937., 1995.

77. Katakam PV, Jordan JE, Snipes JA, Tulbert CD, Miller AW, and Busija DW. Myocardial preconditioning against ischemia-reperfusion injury is abolished 
in Zucker obese rats with insulin resistance. Am J Physiol Regul Integr Comp Physiol 292: R920-926, 2007.

78. Kehrer JP. The Haber-Weiss reaction and mechanisms of toxicity.

Toxicology 149: 43-50, 2000.

79. Kim JS, He L, and Lemasters JJ. Mitochondrial permeability transition: a common pathway to necrosis and apoptosis. Biochem Biophys Res Commun 304: 463-470, 2003.

80. Kinsey GR, McHowat J, Patrick KS, and Schnellmann RG. Role of $\mathrm{Ca} 2+-$ independent phospholipase A2gamma in Ca2+-induced mitochondrial permeability transition. J Pharmacol Exp Ther 321: 707-715, 2007.

81. Korge $\mathbf{P}$, Honda HM, and Weiss $\mathbf{J N}$. Effects of fatty acids in isolated mitochondria: implications for ischemic injury and cardioprotection. Am J Physiol 285: H259-269, 2003.

82. Korichneva I, Hoyos B, Chua R, Levi E, and Hammerling U. Zinc release from protein kinase $C$ as the common event during activation by lipid second messenger or reactive oxygen. J Biol Chem 277: 44327-44331, 2002.

83. Kozlov AV, Staniek K, HaindI S, Piskernik C, Ohlinger W, Gille L, Nohl H, Bahrami S, and RedI H. Different effects of endotoxic shock on the respiratory function of liver and heart mitochondria in rats. Am J Physiol Gastrointest Liver Physiol 290: G543-549, 2006.

84. Krenz M, Oldenburg O, Wimpee H, Cohen MV, Garlid KD, Critz SD, Downey JM, and Benoit JN. Opening of ATP-sensitive potassium channels causes generation of free radicals in vascular smooth muscle cells. Basic Res Cardiol 97: 365-373., 2002.

85. Laclau MN, Boudina S, Thambo JB, Tariosse L, Gouverneur G, Bonoron-Adele S, Saks VA, Garlid KD, and Dos Santos P. Cardioprotection by ischemic preconditioning preserves mitochondrial function and functional coupling between adenine nucleotide translocase and creatine kinase. J Mol Cell Cardiol 33: 947-956, 2001.

86. Lebuffe G, Schumacker PT, Shao ZH, Anderson T, Iwase H, and Vanden Hoek TL. ROS and NO trigger early preconditioning: relationship to mitochondrial K $\mathrm{ATP}_{\mathrm{P}}$ channel. Am J Physiol 284: H299-308, 2003.

87. Lemasters JJ. Integration of mitochondrial function. Plenum Press, 1988.

88. Lesnefsky EJ, and Hoppel CL. Cardiolipin as an oxidative target in cardiac mitochondria in the aged rat. Biochim Biophys Acta 1777: 1020-1027, 2008.

89. Lesnefsky EJ, Slabe TJ, Stoll MS, Minkler PE, and Hoppel CL. Myocardial ischemia selectively depletes cardiolipin in rabbit heart subsarcolemmal mitochondria. Am J Physiol 280: H2770-2778, 2001.

90. Liu GS, Thornton J, Van Winkle DM, Stanley AW, Olsson RA, and Downey JM. Protection against infarction afforded by preconditioning is mediated by $\mathrm{A} 1$ adenosine receptors in rabbit heart. Circulation 84: 350-356, 1991.

91. Liu Y, Yang XM, Iliodromitis EK, Kremastinos DT, Dost T, Cohen MV, and Downey JM. Redox signaling at reperfusion is required for protection from 
ischemic preconditioning but not from a direct PKC activator. Basic Res Cardiol 103: 54-59, 2008.

92. Lopaschuk GD, Folmes CD, and Stanley WC. Cardiac energy metabolism in obesity. Circ Res 101: 335-347, 2007.

93. Mancuso DJ, Jenkins CM, Sims HF, Cohen JM, Yang J, and Gross RW. Complex transcriptional and translational regulation of iPLAgamma resulting in multiple gene products containing dual competing sites for mitochondrial or peroxisomal localization. Eur J Biochem 271: 4709-4724, 2004.

94. McCullough JR, Normandin DE, Conder ML, Sleph PG, Dzwonczyk S, and Grover GJ. Specific block of the anti-ischemic actions of cromakalim by sodium 5-hydroxydecanoate. Circulation Research 69: 949-958, 1991.

95. Mickle DA, and Weisel RD. Future directions of vitamin $E$ and its analogues in minimizing myocardial ischemia-reperfusion injury. The Canadian journal of cardiology 9: 89-93, 1993.

96. Mironova GD, Fedotcheva NI, Makarov PR, Pronevich LA, and Mironov GP. [Protein from beef heart mitochondria inducing the potassium channel conductivity of bilayer lipid membrane]. Biophysic (USSR) 26: 458-465, 1981.

97. Mitchell P. Chemiosmotic coupling in oxidative and photosynthetic phosphorylation. Biol Rev Camb Philos Soc 41: 445-502., 1966.

98. Mitchell P. Coupling of phosphorylation to electron and hydrogen transfer by a chemiosmotic type of mechanism. Nature 191: 144-148, 1961.

99. Mitsos SE, Askew TE, Fantone JC, Kunkel SL, Abrams GD, Schork A, and Lucchesi BR. Protective effects of $\mathrm{N}$-2-mercaptopropionyl glycine against myocardial reperfusion injury after neutrophil depletion in the dog: evidence for the role of intracellular-derived free radicals. Circulation 73: 1077-1086, 1986.

100. Murphy E, Glasgow W, Fralix T, and Steenbergen C. Role of lipoxygenase metabolites in ischemic preconditioning. Circ Res 76: 457-467, 1995.

101. Murphy MP. How mitochondria produce reactive oxygen species. Biochem J 417: 1-13, 2009.

102. Murry CE, Jennings RB, and Reimer KA. Preconditioning with ischemia: a delay of lethal cell injury in ischemic myocardium. Circulation 74: 1124-1136, 1986.

103. Murry CE, Richard VJ, Jennings RB, and Reimer KA. Preconditioning with ischemia: is the protective effect mediated by free radical-induced myocardial stunning? Circulation 78: 77, 1988.

104. Muscari C, Giordano E, Bonafe F, Govoni M, Pasini A, and Guarnieri

C. Priming adult stem cells by hypoxic pretreatments for applications in regenerative medicine. Journal of biomedical science 20: 63, 2013.

105. Nadtochiy SM, Baker PR, Freeman BA, and Brookes PS. Mitochondrial nitroalkene formation and mild uncoupling in ischaemic preconditioning: implications for cardioprotection. Cardiovasc Res 82: 333-340, 2009. 106. Nakamura Y, Miura T, Nakano A, Ichikawa Y, Yano T, Kobayashi H, Ikeda Y, Miki T, and Shimamoto K. Role of microtubules in ischemic 
preconditioning against myocardial infarction. Cardiovasc Res 64: 322-330, 2004.

107. Nobes CD, Brown GC, Olive PN, and Brand MD. Non-ohmic proton conductance of the mitochondrial inner membrane in hepatocytes. Journal of Biological Chemistry 265: 12903-12909, 1990.

108. Obata T, and Yamanaka Y. Block of cardiac ATP-sensitive $\mathrm{K}^{+}$channels reduces hydroxyl radicals in the rat myocardium. Arch Biochem Biophys 378: 195-200., 2000.

109. Oldenburg O, Qin Q, Krieg T, Yang X-M, Philipp S, Critz SD, Cohen MV, and Downey JM. Bradykinin induces mitochondrial ROS generation via NO, cGMP, PKG, and mitoK $\mathrm{ATP}_{\mathrm{TP}}$ channel opening and leads to cardioprotection. Am J Physiol 286: H468-476, 2004.

110. Oldenburg O, Yang XM, Krieg T, Garlid KD, Cohen MV, Grover GJ, and Downey JM. P1075 opens mitochondrial $\mathrm{K}_{\text {ATP }}$ channels and generates reactive oxygen species resulting in cardioprotection of rabbit hearts. $J$ Mol Cell Cardiol 35: 1035-1042., 2003.

111. Pain T, Yang XM, Critz SD, Yue Y, Nakano A, Liu GS, Heusch G, Cohen MV, and Downey JM. Opening of mitochondrial $\mathrm{K}_{\text {ATP }}$ channels triggers the preconditioned state by generating free radicals. Circ Res 87: 460-466, 2000. 112. Paradies G, Petrosillo G, Pistolese M, Di Venosa N, Serena D, and Ruggiero FM. Lipid peroxidation and alterations to oxidative metabolism in mitochondria isolated from rat heart subjected to ischemia and reperfusion. Free Radic Biol Med 27: 42-50, 1999.

113. Pasdois $\mathbf{P}$, Beauvoit B, Tariosse L, Vinassa B, Bonoron-Adele S, and Dos Santos $\mathbf{P}$. Effect of diazoxide on flavoprotein oxidation and reactive oxygen species generation during ischemia-reperfusion: a study on Langendorffperfused rat hearts using optic fibers. Am J Physiol 294: H2088-2097, 2008. 114. Pasdois $\mathbf{P}$, Beauvoit B, Tariosse L, Vinassa B, Bonoron-Adele S, and Santos PD. MitoK(ATP)-dependent changes in mitochondrial volume and in complex II activity during ischemic and pharmacological preconditioning of Langendorff-perfused rat heart. J Bioenerg Biomembr 38: 101-112, 2006. 115. Pasdois PP, Quinlan CL, Rissa A, Tariosse L, Vinassa B, Pierre SV, Dos Santos P, and Garlid KD. Ouabain protects rat hearts against ischemia-

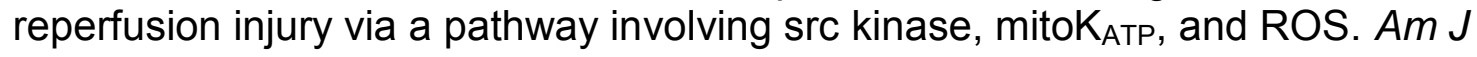
Physiol 292: H1470-1478, 2007.

116. Paucek P, Mironova G, Mahdi F, Beavis AD, Woldegiorgis G, and Garlid KD. Reconstitution and partial purification of the glibenclamide-sensitive, ATP-dependent $\mathrm{K}^{+}$channel from rat liver and beef heart mitochondria. $\mathrm{J}$ Biol Chem 267: 26062-26069, 1992.

117. Pirro M, Mauriege P, Tchernof A, Cantin B, Dagenais GR, Despres JP, and Lamarche B. Plasma free fatty acid levels and the risk of ischemic heart disease in men: prospective results from the Quebec Cardiovascular Study. Atherosclerosis 160: 377-384, 2002. 
118. Prasad MR, Popescu LM, Moraru, II, Liu XK, Maity S, Engelman RM, and Das DK. Role of phospholipases A2 and $\mathrm{C}$ in myocardial ischemic reperfusion injury. Am J Physiol 260: H877-883, 1991.

119. Queliconi BB, Wojtovich AP, Nadtochiy SM, Kowaltowski AJ, and Brookes PS. Redox regulation of the mitochondrial K(ATP) channel in cardioprotection. Biochim Biophys Acta 1813: 1309-1315, 2011.

120. Quinlan CL, Costa ADT, Costa CL, Pierre SV, Dos Santos P, and Garlid KD. Conditioning the Heart Induces Formation of Signalosomes that Interact with Mitochondria to Open MitoK ATP. Am J Physiol 295: H953-961, 2008. 121. Radi R, Turrens JF, Chang LY, Bush KM, Crapo JD, and Freeman BA. Detection of catalase in rat heart mitochondria. J Biol Chem 266: 22028-22034, 1991.

122. Rostovtseva T, and Colombini M. VDAC channels mediate and gate the flow of ATP: implications for the regulation of mitochondrial function. Biophys $\mathrm{J}$ 72: 1954-1962, 1997.

123. Saks VA, Veksler VI, Kuznetsov AV, Kay L, Sikk P, Tiivel T, Tranqui L, Olivares J, Winkler K, Wiedemann F, and Kunz WS. Permeabilized cell and skinned fiber techniques in studies of mitochondrial function in vivo. Mol Cell Biochem 184: 81-100., 1998.

124. Sargent CA, Wilde MW, Dzwonczyk S, Tuttle JG, Murray HN, Atwal K, and Grover GJ. Glyburide-reversible cardioprotective effects of calciumindependent phospholipase A2 inhibition in ischemic rat hearts. Cardiovascular Research 31: 270-277, 1996.

125. Sato T, O'Rourke B, and Marbán E. Modulation of mitochondrial ATPdependent $\mathrm{K}^{+}$channels by protein kinase C. Circ Res 83: 110-114, 1998.

126. Schlattner U, Dolder M, Wallimann T, and Tokarska-Schlattner M. Mitochondrial Creatine Kinase and Mitochondrial Outer Membrane Porin Show a Direct Interaction That Is Modulated by Calcium. J Biol Chem 276: 48027-48030., 2001.

127. Schlattner U, and Wallimann T. Octamers of mitochondrial creatine kinase isoenzymes differ in stability and membrane binding. J Biol Chem 275: 17314-17320., 2000.

128. Schultz JE, Rose E, Yao Z, and Gross GJ. Evidence for involvement of opioid receptors in ischemic preconditioning in rat hearts. Am J Physiol 268: H2157-2161, 1995.

129. Scorrano L, Penzo D, Petronilli V, Pagano F, and Bernardi P. Arachidonic acid causes cell death through the mitochondrial permeability transition. Implications for tumor necrosis factor-alpha aopototic signaling. J Biol Chem 276: 12035-12040, 2001.

130. Smani T, Zakharov SI, Leno E, Csutora P, Trepakova ES, and Bolotina VM. Ca2+-independent phospholipase A2 is a novel determinant of store-operated Ca2+ entry. J Biol Chem 278: 11909-11915, 2003.

131. Spencer KT, Lindower PD, Buettner GR, and Kerber RE. Transition metal chelators reduce directly measured myocardial free radical production during reperfusion. J Cardiovasc Pharmacol 32: 343-348, 1998. 
132. Stanley BA, Sivakumaran V, Shi S, McDonald I, Lloyd D, Watson WH, Aon MA, and Paolocci N. Thioredoxin reductase-2 is essential for keeping low levels of $\mathrm{H}(2) \mathrm{O}(2)$ emission from isolated heart mitochondria. J Biol Chem 286: 33669-33677, 2011.

133. Sultan A, and Sokolove PM. Free fatty acid effects on mitochondrial permeability: an overview. Arch Biochem Biophys 386: 52-61., 2001.

134. Takekoshi S, Nagata H, Kentaro M, and Osamura RY. Involvement of lipid peroxidation in the alteration of protein kinase $\mathrm{C}$ signaling. Acta Histochem Cytochem 36: 281-285, 2003.

135. Tanaka M, Fujiwara H, Yamasaki K, and Sasayama S. Superoxide dismutase and $\mathrm{N}$-2-mercaptopropionyl glycine attenuate infarct size limitation effect of ischaemic preconditioning in the rabbit. Cardiovasc Res 28: 980-986, 1994.

136. Tedeschi $\mathbf{H}$, and Harris $\mathbf{D L}$. The osmotic behavior and permeability to non-electrolytes of mitochondria. Arch Biochem Biophys 58: 52-67, 1955.

137. Teng RJ, Ye YZ, Parks DA, and Beckman JS. Urate produced during hypoxia protects heart proteins from peroxynitrite-mediated protein nitration. Free Radic Biol Med 33: 1243-1249, 2002.

138. Tian J, Liu J, Garlid KD, Shapiro JI, and Xie Z. Involvement of Mitogenactivated Protein Kinases and Reactive Oxygen Species in the Inotropic Action of Ouabain on Cardiac Myocytes. A Potential Role for Mitochondrial KATP Channels. Mol Cell Biochem 242: 181-187, 2003.

139. Tritto I, D'Andrea D, Eramo N, Scognamiglio A, De Simone C, Violante A, Esposito A, Chiariello M, and Ambrosio G. Oxygen radicals can induce preconditioning in rabbit hearts. Circ Res 80: 743-748., 1997.

140. Tsang A, Hausenloy DJ, Mocanu MM, and Yellon DM.

Postconditioning: a form of "modified reperfusion" protects the myocardium by activating the phosphatidylinositol 3-kinase-Akt pathway. Circ Res 95: 230-232, 2004.

141. Turrens JF. Mitochondrial formation of reactive oxygen species. $J$ Physiol 552: 335-344, 2003.

142. Van der Vusse GJ, Reneman RS, and van Bilsen M. Accumulation of arachidonic acid in ischemic/reperfused cardiac tissue: possible causes and consequences. Prostaglandins Leukot Essent Fatty Acids 57: 85-93, 1997.

143. Van der Vusse GJ, Roemen TH, Prinzen FW, Coumans WA, and Reneman RS. Uptake and tissue content of fatty acids in dog myocardium under normoxic and ischemic conditions. Circ Res 50: 538-546, 1982.

144. Van Winkle DM, Thornton JD, Downey DM, and Downey JM. The natural history of preconditioning: cardioprotection depends on duration of transient ischemia and time to subsequent ischemia. Coron Artery Dis 2: 613619, 1991.

145. Vanden Hoek TL, Becker LB, Shao Z, Li C, and Schumacker PT.

Reactive oxygen species released from mitochondria during brief hypoxia induce preconditioning in cardiomyocytes. J Biol Chem 273: 18092-18098, 1998. 
146. Wall TM, Sheehy $\mathbf{R}$, and Hartman JC. Role of bradykinin in myocardial preconditioning. J Pharmacol Exp Ther 270: 681-689, 1994.

147. Wallimann T, Dolder M, Schlattner U, Eder M, Hornemann T, O'Gorman E, Ruck A, and Brdiczka D. Some new aspects of creatine kinase (CK): compartmentation, structure, function and regulation for cellular and mitochondrial bioenergetics and physiology. Biofactors 8: 229-234, 1998.

148. Weiss JN, Korge P, Honda HM, and Ping P. Role of the mitochondrial permeability transition in myocardial disease. Circ Res 93: 292-301, 2003. 149. Williams SD, and Gottlieb RA. Inhibition of mitochondrial calciumindependent phospholipase A2 (iPLA2) attenuates mitochondrial phospholipid loss and is cardioprotective. Biochem J 362: 23-32, 2002.

150. Yaguchi Y, Satoh H, Wakahara N, Katoh H, Uehara A, Terada H, Fujise $\mathbf{Y}$, and Hayashi $\mathbf{H}$. Protective effects of hydrogen peroxide against ischemia/reperfusion injury in perfused rat hearts. Circ J 67: 253-258, 2003. 151. Yao Z, and Gross GJ. Effects of the KATP channel opener bimakalim on coronary blood flow, monophasic action potential duration, and infarct size in dogs. Circulation 89: 1769-1775, 1994.

152. Ytrehus K, Liu Y, Tsuchida A, Miura T, Liu GS, Yang XM, Herbert D, Cohen MV, and Downey JM. Rat and rabbit heart infarction: effects of anesthesia, perfusate, risk zone, and method of infarct sizing. Am J Physiol 267: H2383-2390, 1994.

153. Ytrehus K, Walsh RS, Richards SC, and Downey JM. Hydrogen peroxide as a protective agent during reperfusion. A study in the isolated perfused rabbit heart subjected to regional ischemia. Cardiovasc Res 30: 10331037, 1995.

154. Zhang HY, McPherson BC, Liu H, Baman TS, Rock P, and Yao Z. $\mathrm{H}_{2} \mathrm{O}_{2}$ opens mitochondrial $\mathrm{K}_{\text {ATP }}$ channels and inhibits GABA receptors via protein kinase C-epsilon in cardiomyocytes. Am J Physiol 282: H1395-1403, 2002. 155. Zhao ZQ, Corvera JS, Halkos ME, Kerendi F, Wang NP, Guyton RA, and Vinten-Johansen $\mathbf{J}$. Inhibition of myocardial injury by ischemic postconditioning during reperfusion: comparison with ischemic preconditioning. $\mathrm{Am} \mathrm{J}$ Physiol 3: 3, 2003. 


\section{Appendix A: Publishing Permission}

Chapter 2 "Mitochondrial Reactive Oxygen Species (ROS): Which ROS Signals Cardioprotection?" has been published in the American Journal of Physiology: Heart and Circulatory Physiology section, and the full citation is found in the References section of this thesis, as follows:

36. Garlid AO, Jaburek M, Jacobs JP, and Garlid KD. Mitochondrial Reactive Oxygen Species - Which ROS Signals Cardioprotection? Am J Physiol Heart Circ Physiol 2013.

The "Rights of Authors of APS Articles" states that whole articles from APS journals may be reproduced without charge in dissertations and posted to thesis repositories with full citation.

"Rights of Authors of APS Articles

For educational purposes only, authors may make copies of their own articles or republish parts of these articles (e.g., figures, tables), without charge and without requesting permission, provided that full acknowledgement of the source is given in the new work. Authors may not post a PDF of their published article on any website; instead, links may be posted to the article on the APS journal website.

Posting of articles or parts of articles is restricted and subject to the conditions below:

- Theses and dissertations. APS permits whole published articles to be reproduced without charge in dissertations and posted to thesis repositories. Full citation is required."

\section{http://www.the-aps.org/mm/Publications/Info-For-Authors/Copyright}

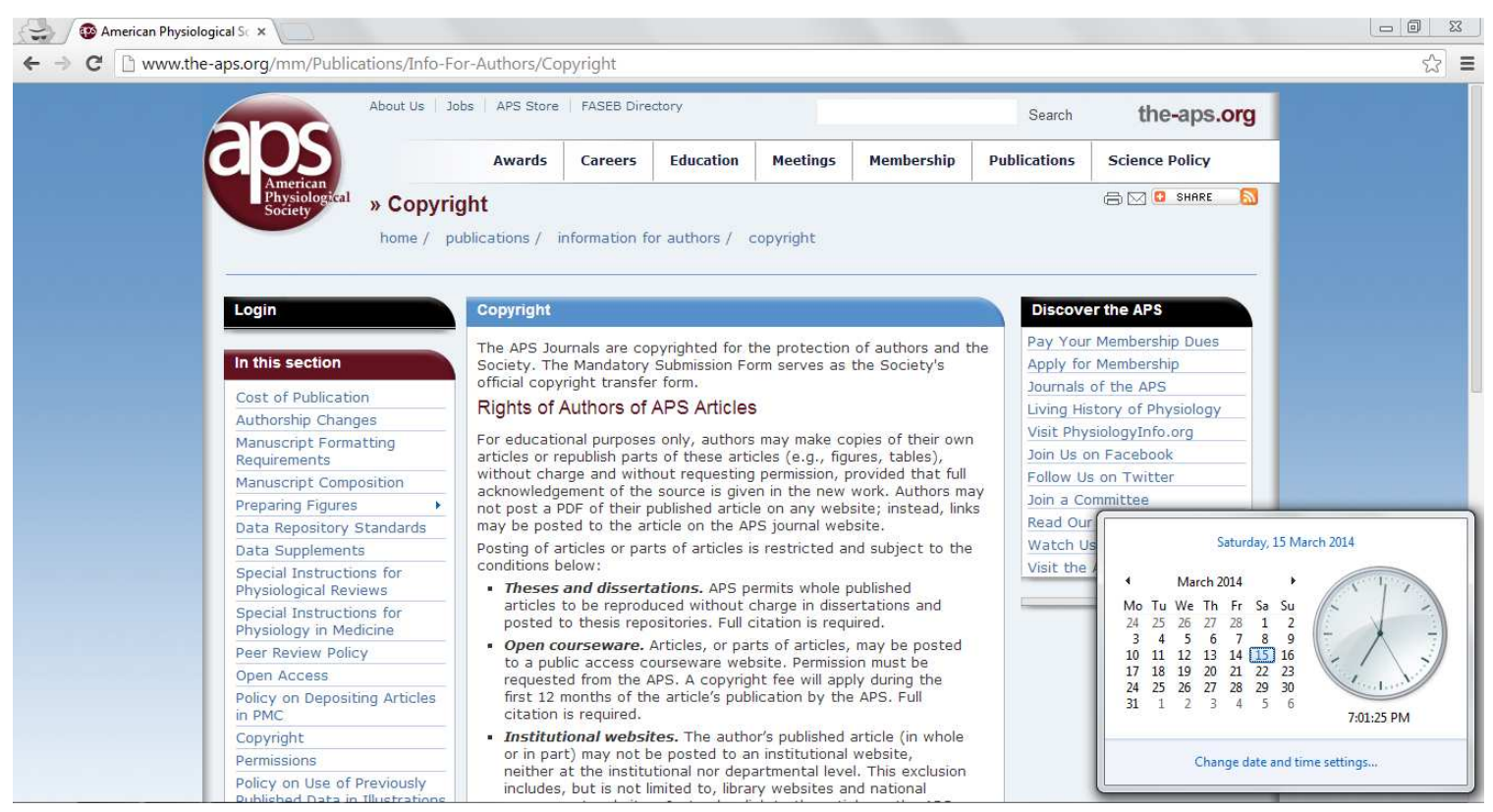

